University of South Carolina

Scholar Commons

Theses and Dissertations

2017

\title{
On Slowly Rotating Supercompact Schwarzschild Stars
}

Nelson Camilo Posada-Aguirre

University of South Carolina

Follow this and additional works at: https://scholarcommons.sc.edu/etd

Part of the Physics Commons

\section{Recommended Citation}

Posada-Aguirre, N. C.(2017). On Slowly Rotating Supercompact Schwarzschild Stars. (Doctoral dissertation). Retrieved from https://scholarcommons.sc.edu/etd/4233

This Open Access Dissertation is brought to you by Scholar Commons. It has been accepted for inclusion in Theses and Dissertations by an authorized administrator of Scholar Commons. For more information, please contact digres@mailbox.sc.edu. 
ON SLOWLY ROTATING SUPERCOMPACT SCHWARZSChILD STARS

by

Nelson Camilo Posada-Aguirre

Licenciado

Universidad Pedagógica Nacional, 2005

Master of Science

Universidad de los Andes, 2009

Master of Science

University of South Carolina, 2014

Submitted in Partial Fulfillment of the Requirements

for the Degree of Doctor of Philosophy in

Physics

College of Arts and Sciences

University of South Carolina

2017

Accepted by:

Pawel O. Mazur, Major Professor

Timir Datta, Committee Member

Matthias Schindler, Committee Member

Daniel Dix, Committee Member

Cheryl L. Addy, Vice Provost and Dean of the Graduate School 
(C) Copyright by Nelson Camilo Posada-Aguirre, 2017 All Rights Reserved. 


\section{ACKNOWLEDGMENTS}

I am grateful to the universe, for its harmony.

I am indebted to the Department of Physics and Astronomy at USC for all the support, in all manners, during these six years.

I want to thank especially Prof. Pawel O. Mazur for accepting me as his student. The fact that someone like me had the opportunity to work with someone like him, is a privilege that very few people have where I come from. His way of thinking of Physics, following the steps of Einstein, Dirac, and Landau, is a role model to be followed.

I want to thank the members of my committee, Dr. Schindler and Dr. Dix for their valuable comments on the manuscript. I want to thank especially Dr. Datta for valuable discussions and suggestions during the preparation of the paper.

Even though I do not know them in person (sadly), I am indebted with Dr. Martin Urbanec and Prof. John C. Miller for invaluable discussions on the numerical part.

To my friends, those near and those far, for the laughs and moments.

To Yohanna Mejía, she knows why.

Foremost, to my mother for her unconditional love and support. 


\begin{abstract}
In this Ph.D thesis, I will present results concerning to my doctoral research project submitted to the Department of Physics and Astronomy at the University of South Carolina. The thesis belongs to the area of Theoretical Physics, particularly, in the framework of Einstein's Theory of General Relativity.

The project is the study of integral and surface properties of slowly rotating homogeneous masses in the gravastar limit $R \rightarrow R_{s}$, where $R_{s}$ is the Schwarzschild radius. For this purpose we followed the perturbative method proposed by Hartle in 1967. In this model, the relativistic equations of structure for a slowly rotating star were derived at second order in the angular velocity $\Omega$. An interesting, and educational, application of this model was investigated by Chandrasekhar and Miller. In their approach, they solved numerically the structure equations of a homogeneous star (constant energy density) up to the Buchdahl bound $(9 / 8) R_{s}$. Based on this work, our objective was to investigate the interesting region below the Buchdahl bound $R_{s}<R<(9 / 8) R_{s}$, which has not been studied previously in the literature. Our results were astonishing. We found that the surface properties and quadrupole mass moment approach the values corresponding to those of the Kerr metric when expanded at second order in angular momentum. This remarkable result provides a long sought solution to the problem of the source of rotation in the Kerr spacetime.
\end{abstract}




\section{TABle of Contents}

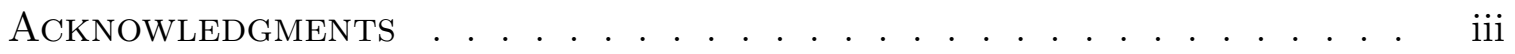

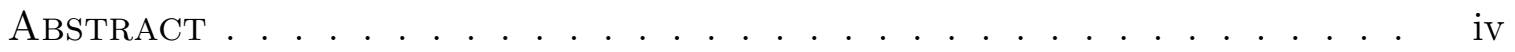

LIST OF TABLES . . . . . . . . . . . . . . . . . vii

List of FigURES . . . . . . . . . . . . . . . . . . . . viii

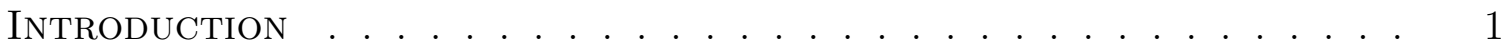

Chapter 1 Black Holes: A Brief Review . . . . . . . . . . . 5

1.1 General Relativity in a Nutshell . . . . . . . . . . . . . . . . 7

1.2 Schwarzschild Spacetime . . . . . . . . . . . . . . . . . . . 14

1.3 Gravitational Collapse . . . . . . . . . . . . . . . . . . . . . . . 21

1.4 Rotating black holes . . . . . . . . . . . . . . . . . 23

1.5 More about Black Holes . . . . . . . . . . . . . . . . . . . . . . 29

1.6 Paradoxes and unsolved issues . . . . . . . . . . . . . 33

Chapter 2 Gravitational Vacuum Condensate Stars . . . . . . 36

2.1 Gravitational vacuumlike condensate model . . . . . . . . . . . . . 38

2.2 Schwarzschild interior solution . . . . . . . . . . . . . . . . . 42

2.3 Schwarzschild star in the 'black hole' limit . . . . . . . . . . . . . 46 
Chapter 3 Slowly rotating Relativistic compact objects . . . . 54

3.1 Rotational 'dragging' of inertial frames . . . . . . . . . . . . . . . 55

3.2 Rotational perturbations in mass-energy and pressure . . . . . . . . . 59

$3.3 l=0$ equations - Spherical deformations of the star . . . . . . . 60

$3.4 l=2$ equations - Quadrupole deformations of the star . . . . . . . 63

3.5 Structure equations for the Schwarzschild star . . . . . . . . . . . 67

Chapter 4 Results . . . . . . . . . . . . . . . . . . . . 75

4.1 Surface and integral properties of Schwarzschild stars for $R>(9 / 8) R_{S} \quad 76$

4.2 Surface and integral properties of Schwarzschild stars in the regime $R_{S}<R<(9 / 8) R_{S} \ldots \ldots \ldots \ldots$. . . . . . . . . 84

4.3 Discussion . . . . . . . . . . . . . . . . . . . . . 91

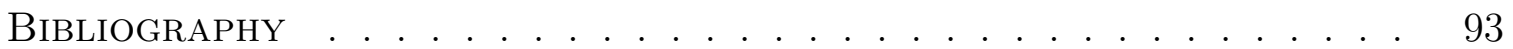

Appendix A Komar integral . . . . . . . . . . . . . . . 101

ApPENDix B EINSTEIN TENSOR FOR AXISYMMETRIC SPACETIMES . . . . . 103

Appendix C Integral AND SURFACE PROperties of A SLOWly ROTATING 'SCHWARZSCHILD STAR' IN GENERAL RELATIVITY . . 106

Appendix D Runge-Kutta methods . . . . . . . . . . . . . 109

D.1 Fourth-order Runge-Kutta method . . . . . . . . . . . . . . . 109

D.2 Adaptive Methods . . . . . . . . . . . . . . . . . 110 


\section{LIST OF TABLES}

Table C.1 Integral and surface properties of a slowly rotating 'Schwarzschild star' for $R>(9 / 8) R_{S} \ldots \ldots \ldots$. . . . . . . . . 107

Table C.2 Integral and surface properties of a slowly rotating 'Schwarzschild star' for $R_{S}<R<(9 / 8) R_{S} \ldots \ldots \ldots 8$ 


\section{List OF Figures}

Figure 1.1 Plot of coordinate and proper time for the Schwarzschild geometry 17

Figure 1.2 Kruskal extension of the Schwarzschild spacetime . . . . . . . . . 20

Figure 1.3 Pictorial diagram of the Kerr geometry . . . . . . . . . . . . . 27

Figure 2.1 Pressure as a function of $\mathrm{r}$ for the Schwarzschild star above the Buchdahl bound ................. . . 45

Figure 2.2 Redshift factor as a function of $\mathrm{r}$ for the Schwarzschild star above the Buchdahl bound . . . . . . . . . . . . . . . 46

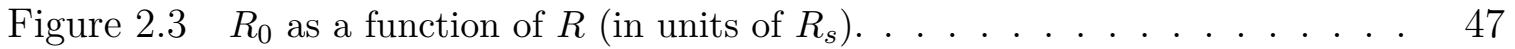

Figure 2.4 Pressure as a function of r, of the interior Schwarzschild solution . 48

Figure 2.5 Redshift factor as a function for the Schwarzschild star below the Buchdahl bound . . . . . . . . . . . . . . 48

Figure 2.6 Metric function $h(r)$ for the gravastar spacetime . . . . . . . . . . 49

Figure 2.7 Pictorial diagram of the Schwarzschild star for $R_{s}<R<(9 / 8) R_{s} \quad 49$

Figure 2.8 Metric function $h(r)$ as a function of $r / R$ for a gravastar . . . . 50

Figure 4.1 Angular velocity $\varpi=\left.(\Omega-\omega)\right|_{r=R}$ relative to the local ZAMO, plotted as a function of the compactness parameter $R / R_{s}$ above the Buchdahl bound . . . . . . . . . . . . . . 78

Figure 4.2 The angular velocity $\Omega$ relative to infinity, plotted as a function of the compactness parameter $R / R_{s}$ above the Buchdahl bound . 79

Figure 4.3 The normalized moment of inertia $I_{N}$ plotted as a function of the compactness parameter $R / R_{s}$ above the Buchdahl bound. . . 79 
Figure 4.4 Fractional change of mass versus the compactness parameter $R / R_{s}$ above the Buchdahl bound $\ldots \ldots \ldots \ldots$

Figure 4.5 The deformation of the star $l=0$ for $R>(9 / 8) R_{S} \ldots \ldots 81$

Figure 4.6 The deformation of the $\operatorname{star} l=2$ for $R>(9 / 8) R_{S} \ldots \ldots 1$

Figure 4.7 Ellipticity of the bounding surface as a function of the compactness parameter $R / R_{s}$ above the Buchdahl bound . . . . . .

Figure 4.8 Ellipticity of the bounding surface as a function of the compactness parameter $R / R_{s}$ above the Buchdahl limit. Horizontal axis plotted with higher resolution $\ldots \ldots \ldots \ldots \ldots$

Figure 4.9 Kerr factor $Q M_{0} / J^{2}$ as a function of the compactness parameter $R / R_{s}$, for $R$ above the Schwarzschild-Buchdahl limit . . . . . .

Figure 4.10 Angular velocity $\varpi=\left.(\Omega-\omega)\right|_{r=R}$ relative to the local ZAMO, plotted as a function of the compactness parameter $R / R_{s}$ for $R_{S}<R<(9 / 8) R_{S} \ldots \ldots \ldots \ldots \ldots$

Figure 4.11 Angular velocity $\Omega(R)$ relative to a distant observer, plotted as a function of the compactness parameter $R / R_{s}$ for $R_{S}<R<$ $(9 / 8) R_{S} \ldots \ldots \ldots \ldots \ldots \ldots$

Figure 4.12 The normalized moment of inertia plotted as a function of the compactness parameter $R / R_{s}$ in the regime $R_{S}<R<(9 / 8) R_{S}$.

Figure 4.13 The original and amended change of mass as a function of the compactness $R / R_{s}$, for Schwarzschild stars in the regime $R_{S}<$ $R<(9 / 8) R_{S} \ldots \ldots \ldots \ldots \ldots \ldots \ldots \ldots \ldots \ldots \ldots$

Figure 4.14 The deformation of the star $l=0$ for $R_{S}<R<(9 / 8) R_{S} \ldots \ldots 89$

Figure 4.15 The deformation of the star $l=2$ for $R_{S}<R<(9 / 8) R_{S} \ldots \ldots 90$

Figure 4.16 Ellipticity of the bounding surface as a function of the compactness parameter $R / R_{s}$, for $R_{S}<R<(9 / 8) R_{S} \ldots \ldots \ldots$

Figure 4.17 Kerr factor $Q M_{0} / J^{2}$ as a function of the compactness parameter $R / R_{s}$, for $R_{S}<R<(9 / 8) R_{S} \ldots \ldots \ldots \ldots$ 


\section{INTRODUCTION}

In classical General Relativity it is commonly accepted that the final state of complete gravitational collapse is a singular state called a 'black hole' $[41,66,102]$. This object is characterized by a central space-time singularity at $r=0$ surrounded by an event horizon, a null hypersurface located at the Schwarzschild radius $r_{S}=2 M$ which separates points connected to infinity by a timelike curve from those that are not. These features are a consequence of the exact solution to Einstein's field equations in the vacuum found a century ago by Karl Schwarzschild [91] which describes the exterior space-time geometry of a spherically symmetric mass.

Despite the vast amount of literature (see e.g. [73, 103] and references therein), the physical reality of black holes has not only generated some skepticism [5, 29, 40], but also has raised some paradoxical issues which have not been consistently solved. A pivotal one is the non-conservation of information by quantum matter falling into a black hole [39]. Additionally in the original Hawking [38] radiation derivation, the backward-in-time propagated mode seems to experience a large blueshift with energies larger than the Planck energy. It is expected that these highly 'blue-shifted' photons would leave a non-negligible 'imprint' on the spacetime geometry, making the approximation of fixed classical geometry background untenable [60,67]. Moreover, the arbitrarily large values of entropy at $T_{H} \rightarrow 0$ associated with the black hole as predicted by the Bekenstein [9] formula in the classical limit $\hbar \rightarrow 0$ produces serious challenges to the foundations of quantum mechanics. It is believed that the resolution of these issues will be achieved in the framework of a consistent theory of quantum gravity. We still do not posses such a theory, therefore it is valuable to investigate 
alternative solutions to the aforementioned problems.

Alternatives have been introduced to alleviate some of the black hole paradoxes $[10,22,96]$. In particular, we concentrate in the gravastar (vacuum condensate gravitational star) model proposed by Mazur and Mottola [60, 61, 62]. A gravastar is basically the aftermath of the gravitational collapse of a star to the Schwarzschild radius $R_{s}$, leaving a final state characterized by a modified de Sitter interior region with negative pressure and a finite surface tension. The exterior spacetime remains the standard spherically symmetric Schwarzschild exterior solution.

In connection with the gravastar, Mazur and Mottola [63] considered the constant density Schwarzschild interior solution, or 'Schwarzschild star'. It is well known that this interior solution shows a divergence in pressure when the radius of the star contracts to $R=(9 / 4) M$, known as the Schwarzschild-Buchdahl bound [13, 92]. The existence of this limit in addition to the homogeneous mass approximation, considered 'unrealistic', have been assumed to be sufficient reasons to exclude the Schwarzschild star from further investigation [102]. This complete disregard of the interior solution has left the interesting region $R_{s}<R<(9 / 8) R_{s}$ unexplored.

In a bold approach Mazur and Mottola [63] analyzed this 'forbidden' region and found that the divergence in the central pressure is integrable through the Komar formula [50], producing a $\delta$-function of transverse stresses implying a relaxation of the isotropic fluid condition on a surface of some radius $R_{0}$. In the limit when $R \rightarrow R_{s}^{+}$ from above and $R_{0} \rightarrow R_{s}^{-}$from below, the interior region suffers a phase transition (starting at the centre) becoming one of negative pressure evoking a de Sitter spacetime. This non-singular 'bubble' of dark energy which is matched to an external vacuum Schwarzschild spacetime, has zero entropy and temperature, so providing a consistent picture of a gravitational Einstein-Bose condensate, or gravastar, as the final state of complete gravitational collapse.

The relevance of gravastars follows from the fact that their physical properties 
and behaviour are governed by classical general relativity. Gravastars are being recognized as a very challenging alternative to black holes. Moreover, calculations of observational consequences of a merger of either two black holes or two gravastars in the context of gravitational waves, e.g. ringdowns [23] and afterglows [4], may provide methods to discriminate between black holes and gravastars.

Some authors [54] have investigated possible sources for the interior of the gravastar, and the electrically charged case was considered by [44]. The issue of stability against axial perturbations was studied by [24]. They found that gravastars are stable under axial perturbations, moreover, the quasi-normal modes of rotating gravastars deviate from those associated with a black hole. They concluded that this might help to distinguish observationally between a gravastar and a black hole. Radial and axial gravitational perturbations on thin-shell gravastars were studied by [75, 76].

Perturbation theory can also be applied to the study of equilibrium configurations of slowly rotating compact objects. In a seminal paper Hartle [33] provided the relativistic structure equations to determine the equilibrium configurations of slowly rotating stars to second order in the angular velocity. In Hartle's model the interior of the star is composed of a fluid characterized by a general one-parameter equation of state (EOS). This configuration is matched to a stationary and axially symmetric exterior region across a timelike hypersurface.

Chandrasekhar and Miller [21] studied slowly rotating homogeneous masses characterized by a constant energy density, using Hartle's framework. For this configuration they solved numerically the structure equations for several values of the parameter $R / R_{s}$ where $R$ is the radius of the star and $R_{s}$ is the Schwarzschild radius. Using these solutions Chandrasekhar \& Miller calculated integral and surface equilibrium properties such as moment of inertia and mass quadrupole moment up to the Buchdahl bound. They found that the ellipticity of the star, considering constant mass and angular momentum, manifests a prominent maximum at the radius $R / R_{s} \sim 2.4$. 
One result of particular interest is that for a star with the 'minimum possible' radius $R=(9 / 8) R_{s}$, the quadrupole mass moment is very close to the value associated with the Kerr metric to second order in the angular velocity.

Motivated by the aforementioned works, in this paper we report results of surface and integral properties of a slowly rotating Schwarzschild star in the unstudied region $R_{s}<R<(9 / 8) R_{s}$. These results extend those presented by Chandrasekhar and Miller [21] which where considered up to the Buchdahl radius. We show that for a Schwarzschild star in the gravastar limit when $R \rightarrow R_{s}$, surface properties like moment of inertia, angular velocity and mass quadrupole moment approach the corresponding Kerr metric values. These remarkable results provide a long sought solution to the problem of the source of rotation of a slowly rotating Kerr black hole.

This Introduction has been taken from the author's paper [82] 


\section{CHAPTER 1 \\ Black Holes: A BRIEF REVIEW \\ This can't possibly be true... There ought to be a law of nature to prevent stars from behaving in this foolish manner.}

Sir Arthur Eddington

\section{INTRODUCTION}

In 1905 Albert Einstein published the Special Theory of Relativity (SR) [28] which is founded on the special principle of relativity. If we choose a reference system $O$ where the laws of Physics takes their simplest form, the same laws will hold too in a second system $O^{\prime}$ which is in rectilinear and uniform motion relative to $O$. This statement is in harmony with the laws of classical mechanics. On the other hand, the constancy of the speed of light and its character as a natural limit clearly deviates from the precepts of Newtonian physics where there is no limit of velocities. Therefore, it is the unification of the principle of relativity with the finiteness of the speed of light which consolidates the principle of relativity of Einstein [52].

The transition from SR to the General Theory of Relativity (GR) was inspired by the ideas of Mach [55] on the inertial properties of bodies in classical mechanics. Moreover, the problem that Einstein faced was to extend the principle of relativity to systems of reference in any motion [28]. The path for this achievement was elucidated by the particular property of the gravitational field which imparts the same 
acceleration to all bodies, independent of their constituents.

Somehow this resembles the properties of inertial systems where free bodies will move uniformly following straight lines, assuming that they were given the same initial conditions. If this reference frame is now accelerated (non-inertial), these bodies will move in the same way relative to it. This reasoning led Einstein to establish the equivalence principle, namely, a uniformly accelerated reference system is equivalent to a constant uniform gravitational field [52].

A statement which is usually ignored in the literature $[14,90,95,102]$ and only remarked in [52] is that this equivalence between gravitational fields and non-inertial frames is not complete. The difference settles on the behavior at infinity of these fields. In correspondence with the Newtonian limit, the gravitational field vanishes at infinity in contrast with the non-inertial 'fields' which increase or remain finite at this limit. One example of this is the centrifugal force in a rotating reference frame which increases with no limit as we move out from the axis of rotation.

It is clear that, in contrast to the case of non-inertial frames which can be 'eliminated' by simple transformations to an inertial frame, gravitational fields cannot be removed by any choice of reference frame. In other words, there is no way to 'screen out' the effects of a gravitational field. Thus we have an impossibility to construct inertial frames (background observers) as we did for SR. The way how Einstein sorted out the solution to this puzzle was to assume that the spacetime metric is not flat. Free observers in a gravitational field will follow the geodesic of the curved spacetime. Gravity becomes an effect of the spacetime geometry. Nevertheless, if we choose a small region ${ }^{1}$ where the gravitational field can be considered to be uniform, then this field will vanish there.

Considering that GR extends the postulate of relativity, how should we write the laws of Physics, such that, they apply to systems of reference in any kind of motion?.

\footnotetext{
${ }^{1}$ Small compared to the radii of curvature of the source of gravitational field.
} 
The answer is provided by the covariance principle as formulated by Einstein [28]

The general laws of nature are to be expressed by equations which hold good for all systems of co-ordinates, that is, are co-variant with respect to any substitution whatever (generally co-variant).

In other words, the equations of physics must be written covariantly in tensorial form. Therefore, the only spacetime quantities that must appear in the laws of physics are the metric $g_{\mu \nu}$ and quantities derivable from it [102]. Additionally, we require that in the Lorentz spacetime $\eta_{\mu \nu}$ (flat space) the equations must reduce to their corresponding forms in special relativity.

The chapter is organized as follows. In the next section, we summarize the essential formalism of general relativity. The Schwarzschild exterior solution is introduced in section 1.2 where we also discuss its main geometrical properties, including the coordinate singularity at the Schwarzschild radius $r_{S}=2 M$ and the Kruskal extension. In section 1.3 we discuss, in a heuristic manner, the gravitational collapse of stars. The gravitational field due to a spinning mass is presented in section 1.4. In section 1.5 we discuss some general properties of black holes, including the Laws of Black Hole Mechanics and Hawking effect. We close the section, summarizing the powerful Black Hole Uniqueness Theorems. Finally, in section 1.6, we discuss some paradoxical issues which plague the classical theory of black holes.

\subsection{General Relativity in a Nutshell}

Notation and conventions. We follow the conventions of [66]. That is, Greek indices $(\mu, \nu, \alpha, \ldots)$ refer to spacetime coordinates running from 0 to 3 , with 0 indicating the time coordinate. Latin indices $(i, j, k, \ldots)$ refer to three-dimensional space coordinates running from 1 to 3 . The metric signature is taken to be $(-,+,+,+)$ and we follow the Einstein summation convention. Additionally, we use the so-called 
geometric units, where the speed of light $c$ and the Newtonian gravitational constant $G$ are equal to 1 .

\subsubsection{THE SPACETIME MANIFOLD}

In general relativity, the generalization of the interval (measurement of local distances) is given by the quadratic form

$$
d s^{2}=g_{\mu \nu} d x^{\mu} d x^{\nu}
$$

where the function $g_{\mu \nu}$ is called the spacetime metric which, in general, is a function of the coordinates $x^{\mu}$. Thus GR permits the metric $g_{\mu \nu}$ to be curved as a generalization of the flat spacetime of SR determined by the Lorentz metric $\eta_{\mu \nu}$. According to GR, the space-time must be curved where, physically, there is a gravitational field. The relation between the source of the gravitational field (energy) and the geometry of the spacetime will be determined by the Einstein equation which will be discussed later. Thus the mathematical model we will follow for spacetime, which is the collection of all physical events, is a differentiable manifold $\mathcal{M}$ on which a metric $g_{\mu \nu}$ can be defined.

On a manifold $\mathcal{M}$ we define the derivative operator or covariant derivative of a vector $V^{\mu}$ by

$$
\nabla_{\alpha} V^{\beta}=\partial_{\alpha} V^{\beta}+\Gamma_{\beta \gamma}^{\alpha} V^{\gamma}
$$

where $\partial_{\mu} \equiv \frac{\partial}{\partial x^{\mu}}$ and $\Gamma_{\beta \gamma}^{\alpha}$ denotes the Christoffel symbols which, in terms of the partial derivative operator, satisfies

$$
\Gamma_{\alpha \beta}^{\gamma}=\frac{1}{2} g^{\gamma \rho}\left[\partial_{\alpha} g_{\beta \rho}+\partial_{\beta} g_{\alpha \rho}-\partial_{\rho} g_{\alpha \beta}\right]
$$


Given the derivative operator $\nabla_{\alpha}$ we can introduce the concept of parallel transport. Given a curve $C$ with a tangent vector $u^{\alpha}$, we say that a vector $v^{\mu}$ is parallelly transported along the curve $C$ if the equation

$$
u^{\alpha} \nabla_{\alpha} v^{\beta}=0
$$

is satisfied. Notice that due to its tensorial nature, this relation is a frame-invariant definition [90]. In the general relativity spacetime, particles follow geodesics which intuitively corresponds to the 'straightest trajectory' on a curved geometry. More formally, a geodesic is a curve that transports parallelly its tangent vector $V^{\alpha}$ satisfying the condition

$$
V^{\alpha} \nabla_{\alpha} V^{\beta}=0 .
$$

Let us parametrize the curve by some affine parameter $\tau$. The tangent vector is then $V^{\alpha}=d x^{\alpha} / d \tau$ and (1.5) takes the final form

$$
\frac{d^{2} x^{\mu}}{d \tau^{2}}+\Gamma_{\sigma \nu}^{\mu} \frac{d x^{\sigma}}{d \tau} \frac{d x^{\nu}}{d \tau}=0 .
$$

This is the geodesic equation, so given initial values for $x^{\mu}$ and $d x^{\mu} / d \tau$, we can always find a unique solution to (1.6). More formally, given a point $p$ on $\mathcal{M}$ and a tangent vector $V^{\alpha}$ on the tangent space $V^{p}$, there is a unique geodesic through $p$ with tangent $V^{\alpha}$.

The notion of parallel transport also provides a mechanism to define curvature intrinsically. In the free-torsion case, the action of the commutator of $\nabla$ on a vector field $V^{\mu}$ corresponds to

$$
\left[\nabla_{\mu}, \nabla_{\nu}\right] V^{\rho}=R_{\mu \nu \beta}^{\rho} V^{\beta}
$$

where $R_{\mu \nu \beta}^{\rho}$ is the Riemann tensor, or curvature tensor, which in components can be written as 


$$
R_{\sigma \mu \nu}^{\rho}=\partial_{\mu} \Gamma_{\nu \sigma}^{\rho}-\partial_{\nu} \Gamma_{\mu \sigma}^{\rho}+\Gamma_{\mu \lambda}^{\rho} \Gamma_{\nu \sigma}^{\lambda}-\Gamma_{\nu \lambda}^{\rho} \Gamma_{\mu \sigma}^{\lambda} .
$$

As usual, we can use the metric tensor to raise and low indices

$$
R_{\rho \sigma \mu \nu}=g_{\rho \lambda} R_{\sigma \mu \nu}^{\lambda} .
$$

From the definition (1.8), it can be verified that the Riemann tensor is antisymmetric in its first two indices

$$
R_{\rho \sigma \mu \nu}=-R_{\sigma \rho \mu \nu} .
$$

It is also antisymmetric in its last two indices

$$
R_{\rho \sigma \mu \nu}=-R_{\rho \sigma \nu \mu},
$$

and it is symmetric under exchange of the first pair of indices with the second

$$
R_{\mu \nu \rho \sigma}=R_{\rho \sigma \mu \nu} .
$$

The curvature tensor also has the symmetry

$$
R_{\rho \sigma \mu \nu}+R_{\rho \mu \nu \sigma}+R_{\rho \nu \sigma \mu}=0 .
$$

Similarly, the covariant derivative of the curvature tensor satisfies the Bianchi identity

$$
\nabla_{\lambda} R_{\rho \sigma \mu \nu}+\nabla_{\rho} R_{\sigma \lambda \mu \nu}+\nabla_{\sigma} R_{\lambda \rho \mu \nu}=0 .
$$

From the contraction of the Riemann tensor we obtain the Ricci tensor

$$
R_{\mu \nu} \equiv R_{\mu \lambda \nu}^{\lambda},
$$

which is totally symmetric $R_{\mu \nu}=R_{\nu \mu}$ by the symmetries of the Riemann tensor. By contraction of the Ricci tensor, we obtain the Ricci scalar or curvature scalar 


$$
R \equiv g^{\mu \nu} R_{\mu \nu}=R_{\mu}^{\mu},
$$

which corresponds to the trace of the Ricci tensor. From its symmetries, the Riemann tensor has $\frac{1}{12} n^{2}\left(n^{2}-1\right)$ algebraically independent components, where $n$ is the dimension of the space. Finally we can define the Einstein tensor as

$$
G_{\mu \nu} \equiv R_{\mu \nu}-\frac{1}{2} R g_{\mu \nu},
$$

which is symmetric by virtue of the symmetry of the metric and the Ricci tensor. One important property of this tensor is that its covariant derivative vanishes

$$
\nabla^{\mu} G_{\mu \nu}=0
$$

This divergenceless condition of the Einstein tensor is essential for the consistency of the Einstein equations with the energy-momentum conservation.

\subsubsection{MATTER FIELDS}

In Einstein's theory of gravitation, the central idea is that the spacetime metric represents the gravitational field. Moreover, the metric will be determined by the matter fields, such as the electromagnetic field, neutrino field, etc., which describe the matter content in the spacetime [41]. Therefore, urges to make the distinction between gravitational fields and matter fields. In this context, matter includes everything (baryons, leptons, electromagnetic fields, etc.) except gravitational fields [28].

By the general covariance principle, the matter fields obey tensorial equations defined on the manifold $\mathcal{M}$, where spatial derivatives become covariant derivatives whose connection depends on the metric tensor $g_{\mu \nu}$. In other words, the matter fields will be determined by tensor equations which involve the metric. Besides local causality (events must be connected by non-spacelike curves on $\mathcal{M}$ ), the matter fields obey equations such that we can define the energy-momentum tensor $T^{\mu \nu}$, which depends 
on the matter fields, the metric $g_{\mu \nu}$ and derivatives. The energy-momentum tensor is symmetric and vanishes if there are no matter fields on $\mathcal{M}$. By local conservation of energy and momentum we have

$$
\nabla_{\mu} T^{\mu \nu}=0
$$

So far we have postulated some general properties of the energy-momentum tensor. However, we have not discussed a general formalism which would allow us to construct $T^{\mu \nu}$ given some matter fields. There exists the Lagrangian formulation, which provides a method to derive the form of $T^{\mu \nu}$ given some Lagrangian (see e.g. $[41] \S 3.3)$. This formalism is beyond the scope of this section, and we will not discuss it here.

For our immediate purpose, we use the following definition of the energy-momentum tensor, in components in some arbitrary frame $\mathcal{O}[90]$

$$
T^{\mu \nu} \equiv\{\text { flux of } \mu \text { momentum across a surface of constant } \nu\}
$$

Here $\mu$-momentum indicates the $\mu$ component of the four-momentum $p^{\mu} \equiv(E, \vec{p})$, where $p^{0}=E$ is the energy of the particle in $\mathcal{O}$, and $\vec{p}$ is the spatial momentum. For example, for a perfect fluid ${ }^{2}$ the energy-momentum tensor takes the form

$$
T^{\mu \nu}=(\epsilon+p) u^{\mu} u^{\nu}+p g^{\mu \nu}
$$

where $\epsilon$ is the energy density, $p$ is the pressure and $u^{\mu}$ is the four-velocity. Notice that for a pressureless perfect fluid, which can be considered as composed of grains of "dust" which do not interact with each other, equation (1.21) together with the local conservation of energy (1.19) result into the geodesic equation (1.5). Thus,

\footnotetext{
${ }^{2} \mathrm{~A}$ perfect fluid is characterized for having no viscous effects and heat fluxes, and its pressure tensor which is diagonal [86].
} 
Einstein's equations imply that free test particles follow geodesics in the spacetime. This remarkable result shows the self-consistency and beauty of Einstein's theory.

\subsubsection{The Einstein Field Equations}

Following a plausible approach, Einstein proposed the field equations based on the following arguments:

- The equations must be written in tensorial form (covariance principle)

- In the limit of weak fields and low velocities $(v<<c)$, the field equations must reduce to the Newtonian field equation

$$
\nabla^{2} \phi=4 \pi G \rho
$$

where $\phi$ and $\rho$ denotes the gravitational field and mass density, respectively.

- The energy-momentum tensor $T_{\mu \nu}$ corresponds to the source of the gravitational field.

In November 25th of 1915, after 10 years of intensive research, Einstein gave final form to the field equations which relates the geometry of the spacetime with the mass-energy distribution. The Einstein equations reads

$$
R_{\mu \nu}-\frac{1}{2}(R-2 \Lambda) g_{\mu \nu}=\frac{8 \pi G}{c^{4}} T_{\mu \nu}
$$

where $\Lambda$ is the cosmological constant (we show the proportionality constant with its correct $G$ and $c$ dependence). Given fixed initial conditions, the Einstein equations are a system of 10 coupled partial differential equations in the metric $g_{\mu \nu}$ and its derivatives. However the covariant divergence of both sides of (1.23) is identically zero, thus we only have six independent differential equations for the metric $g_{\mu \nu}$.

At first glance, one might think that given the form of $T_{\mu \nu}$ one simply solves for $g_{\mu \nu}$ from (1.23). However $T_{\mu \nu}$ also depends on the metric function $g_{\mu \nu}$, thus in 
general relativity spacetime and matter form a dynamical structure which must be solved simultaneously. Following Wheeler's aphorism: Space acts on matter, telling it how to move. In turn, matter reacts back on space, telling it how to curve.[66]

Even though Einstein introduced the $\Lambda$-term in his paper on cosmological considerations of 1917 [28], he was already aware of the possibility of adding a term in his equations before discussing any ideas on cosmology. In a footnote in his paper of 1916 [28], after he wrote the divergenceless condition of (1.17), Einstein wrote:

Properly speaking, this can be affirmed only of the tensor $G_{\mu \nu}+\lambda g_{\mu \nu} g^{\alpha \beta} G_{\alpha \beta}$, where $\lambda$ is a constant. If, however, we set this tensor $=0$, we come back again to the equations $G_{\mu \nu}=0$.

Why did Einstein decide to ignore this term at that time?. According to him, it "removed" the beauty of the theory. However, this argument was not considered anymore when he put it back again in 1917 in his discussion on cosmology. Beyond the controversy around this "cosmological constant" (see e.g. [11]), the $\Lambda$-term turned out to be relevant in the last decade after the discovery of the accelerated expansion of the universe $[78,87]$. In the accepted picture this term is associated with the "dark energy", an elusive 'substance' which is credited for the accelerated cosmological expansion.

To summarize, in general relativity, spacetime is a Riemannian space on which we define a metric tensor $g_{\mu \nu}$. The curvature of $g_{\mu \nu}$ is related to the mass-energy content $T_{\mu \nu}$ through Einstein's equations.

\subsection{Schwarzschild Spacetime}

Few months after Einstein published his field equations, Karl Schwarzschild [91] found the first exact solution in vacuum $\left(T^{\mu \nu}=0\right)$, which represents the geometry of the 
empty spacetime outside a static and spherically symmetric mass ${ }^{3}$. In coordinates $(t, r, \theta, \phi)$ the Schwarzschild metric is given by

$$
d s^{2}=-\left(1-\frac{2 M}{r}\right) d t^{2}+\left(1-\frac{2 M}{r}\right)^{-1} d r^{2}+r^{2}\left(d \theta^{2}+\sin ^{2} \theta d \phi^{2}\right)
$$

where the parameter $M$ is associated with the mass of the object, as measured at infinity. Note that in the limit when $r \rightarrow \infty$, (1.24) takes the Minkowskian form of the Special Relativity spacetime. This property is called asymptotic flatness. The relevance of the Schwarzschild space-time, besides the three experimental tests suggested by Einstein [14, 66], follows from the fact that it corresponds to the unique solution to Einstein's equations in the vacuum with spherical symmetry, as stated by Birkhoff's theorem [41].

A quick glance of (1.24) shows that near the point $r=r_{S} \equiv 2 M$, commonly known as the Schwarzschild radius, the temporal component $g_{t t}$ vanishes and the radial component $g_{r r}$ blows up. In principle, this anomalous behavior might be due to a pathology of the spacetime geometry itself, or it might be due to an inconvenience in the Schwarzschild coordinates. It is important to recall that the metric elements are coordinate-dependent; therefore, a change in coordinates might alleviate the singular behavior. To analyze the nature of the singularity at the gravitational radius, we compute the Kretschmann curvature scalar from (1.16), which in the case of the metric (1.24), takes the form

$$
R=R^{\mu \nu \rho \sigma} R_{\mu \nu \rho \sigma}=\frac{48 M^{2}}{r^{6}}
$$

which is regular at $r=2 M$, indicating that the geometry is well behaved there. The implications of this result are far reaching. For instance, an observer who is radially-

\footnotetext{
${ }^{3}$ In general, not only the mass distribution, but also the motion of the mass must be centrally symmetric, i.e., the velocity at each point must be directed along the radial direction [52].
} 
falling towards the Schwarzschild radius will not feel infinite tidal forces. Once she crosses $r=2 M$, she would not notice anything special at that point ${ }^{4}$.

The fact that the Schwarzschild metric components are not well behaved at the Schwarzschild radius _ although nothing dramatic happens there with the spacetime geometry_ implies that there is a pathology in the Schwarzschild coordinates. Moreover notice that in the region $r>2 M$, the Killing vector $\partial_{t}$ is timelike $\left(g_{t t}<0\right)$ and $\partial_{r}\left(g_{r r}>0\right)$ is spacelike ${ }^{5}$. However in the region $r<2 M$ there is a reversal in this behavior, namely, $\partial_{t}$ becomes spacelike and $\partial_{r}$ becomes timelike. The volume of the surface at $r=2 M$, where $g_{t t}=0$ and $g_{r r} \rightarrow \infty$, is found to be

$$
\int_{r=2 M}\left|g_{t t} g_{\theta \theta} g_{\phi \phi}\right|^{1 / 2} d t d \theta d \phi=0
$$

which implies that $r=2 M$ is a null surface. Therefore, $r=2 M$ is a two-dimensional hypersurface with area

$$
\int_{r=2 M, t=\text { const. }}\left|g_{\theta \theta} g_{\phi \phi}\right|^{1 / 2} d \theta d \phi=4 \pi(2 M)^{2} .
$$

An extraordinary situation occurs when one analyzes the trajectory of a zero angular momentum observer (ZAMO) in free-fall. The radial coordinate $r$, as a function of the coordinate time, is given by [66]

$$
\frac{t}{2 M}=-\frac{2}{3}\left(\frac{r}{2 M}\right)^{3 / 2}-2\left(\frac{r}{2 M}\right)^{1 / 2}+\ln \left|\frac{(r / 2 M)^{1 / 2}+1}{(r / 2 M)^{1 / 2}-1}\right|+\text { const. }
$$

Similarly once can find the r-coordinate of the free-falling observer as a function of her proper time $\tau$. The function $r(\tau)$ give

\footnotetext{
${ }^{4}$ The tidal forces felt by an observer are determined by the components of the Riemann tensor in his orthonormal frame [66]

${ }^{5} \mathrm{~A}$ vector $K_{\mu}$ which satisfies the Killing equation $\nabla_{\mu} K_{\nu}+\nabla_{\nu} K_{\mu}=0$, is called a Killing vector. In general the existence of Killing vectors imply conserved quantities associated with geodesic motion.
} 


$$
\frac{\tau}{2 M}=-\frac{2}{3}\left(\frac{r}{2 M}\right)^{3 / 2}+\text { const }
$$

Figure 1.1 shows the coordinate and proper time (in units of $M$ ) as a function of $r$ (in units of $M$ ). Notice the bizarre behavior of the free-falling observer's trajectory. According to a distant observer (coordinate time), she takes an infinite amount of time to reach the surface $r=2 M$. However, according to her clock, she takes a finite time to arrive at the gravitational radius, cross it, and eventually end up at the central singularity in $r=0$.

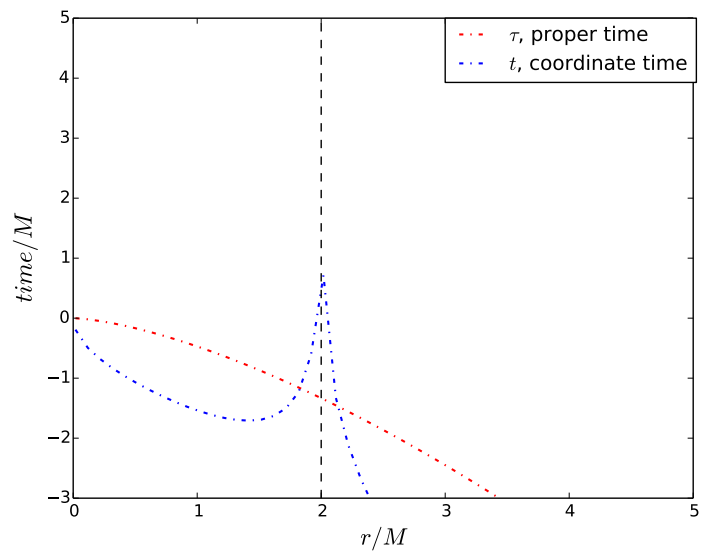

Figure 1.1: Plot of coordinate and proper time (in units of $M$ ), as a function of the radial distance $\mathrm{r}$ (in units of $\mathrm{M}$ ), for the Schwarzschild geometry.

The paradoxical behavior of the Schwarzschild geometry can also be studied through its causal structure, as determined by the light cones. Considering radial null geodesics $(d \theta=d \phi=0)$, from $(1.24)$ we have

$$
d s^{2}=-\left(1-\frac{2 M}{r}\right) d t^{2}+\left(1-\frac{2 M}{r}\right)^{-1} d r^{2}=0
$$

which gives

$$
\frac{d t}{d r}= \pm\left(1-\frac{2 M}{r}\right)^{-1}
$$

Equation (1.31) corresponds to the slope of the light cones on a spacetime diagram. In the Minkowski spacetime $d r / d t= \pm 1$ indicating a flat geometry. Notice that 
(1.31) approaches 1 when $r \rightarrow \infty$, as expected from its asymptotically flat behavior. However, in the limit $r \rightarrow 2 M, d t / d r \rightarrow \infty$ indicating a closing up of light cones near the Schwarzschild radius.

According to this, a distant observer will find that the light signals sent by the ZAMO in free-fall towards $r=2 M$ are redshifted. Moreover, she will find that her partner, who went on that perilous journey, will move slower as time goes. According to (1.28), she will see that her partner never gets to the surface $r=2 M$. However, after a while, she will find that the frequency of the light signals is incredibly low, that she cannot detect them anymore. For the distant observer, her partner just vanished into oblivion.

It is worthwhile to recall that the Schwarzschild solution (1.24) can be applied only outside the configuration $r>R$. In most practical cases the Schwarzschild radius is very small compared to the dimensions of the object. For example, for the Sun $r_{S}=2.96 \mathrm{Km}$ and for the Earth it is $r_{S}=8.8 \mathrm{~mm}$ [95]. Nevertheless in extreme cases, for example when a star suffers gravitational collapse, the full exterior solution must be considered. In this extreme case, it turns out that the surface $r=2 M$ becomes a one-way membrane where particles and radiation can get in but nothing can escape from it. The surface $r=2 M$ is called an event horizon. In more technical terms, en event horizon is a hypersurface which separates points connected to future infinity by timelike curves, from those that are not [41]. Considering that nothing can escape from the event horizon, not even light, it is not possible to detect any signal coming from it. This is the reason why J. A. Wheeler coined the term black hole [104].

\subsubsection{Analytical Extension}

Despite the paradoxical behavior of the Schwarzschild coordinates near the gravitational radius $r=2 M$, the scalar of curvature (1.25) shows that the geometry behaves well there. This fact suggests that a different coordinate system might alleviate the 
issues at the Schwarzschild radius.

One of the simpler coordinate systems that extend the Schwarzschild spacetime was proposed by Kruskal[51] and independently by Szekeres[98]. They introduced coordinates $(u, v)$ which are related to the Schwarzschild coordinates $(r, t)$ through the transformation

$$
\begin{aligned}
& r>2 M\left\{\begin{array}{l}
u=\left(\frac{r}{2 M}-1\right)^{1 / 2} e^{r / 4 M} \cosh (t / 4 M) \\
v=\left(\frac{r}{2 M}-1\right)^{1 / 2} e^{r / 4 M} \sinh (t / 4 M)
\end{array}\right. \\
& r<2 M\left\{\begin{array}{l}
u=\left(1-\frac{r}{2 M}\right)^{1 / 2} e^{r / 4 M} \sinh (t / 4 M) \\
v=\left(1-\frac{r}{2 M}\right)^{1 / 2} e^{r / 4 M} \cosh (t / 4 M)
\end{array}\right.
\end{aligned}
$$

where $u$ corresponds to the radial coordinate and $v$ to the time coordinate. In terms of the coordinates $(u, v)$, the Schwarzschild metric (1.24) takes the form [66]

$$
d s^{2}=\left(\frac{32 M^{3}}{r}\right) e^{-r / 2 M}\left(-d v^{2}+d u^{2}\right)+r^{2}\left(d \theta^{2}+\sin ^{2} d \phi^{2}\right)
$$

where $r$ and $t$ are functions of the coordinates $(u, v)$ and are given by the inverse transformations

$$
\begin{gathered}
\left(\frac{r}{2 M}-1\right) e^{r / 2 M}=u^{2}-v^{2} . \\
t= \begin{cases}4 M \tanh ^{-1}(v / u), & \text { in regions I and III } \\
4 M \tanh ^{-1}(u / v), & \text { in regions II and IV. }\end{cases}
\end{gathered}
$$

In Figure 1.2 the transformations (1.35) and (1.36) are plotted for various values of $r=$ const. and $t=$ const. Notice that in Kruskal coordinates, curves of constant $r$ correspond to hyperbolae and surfaces of constant $t$ are straight lines. An important feature of this analytical extension is that null radial geodesics in Kruskal coordinates, such that $d s=0$, correspond to 45 -degree lines (red lines). This behavior resembles the causal structure of the Minkowski spacetime of Special Relativity. 


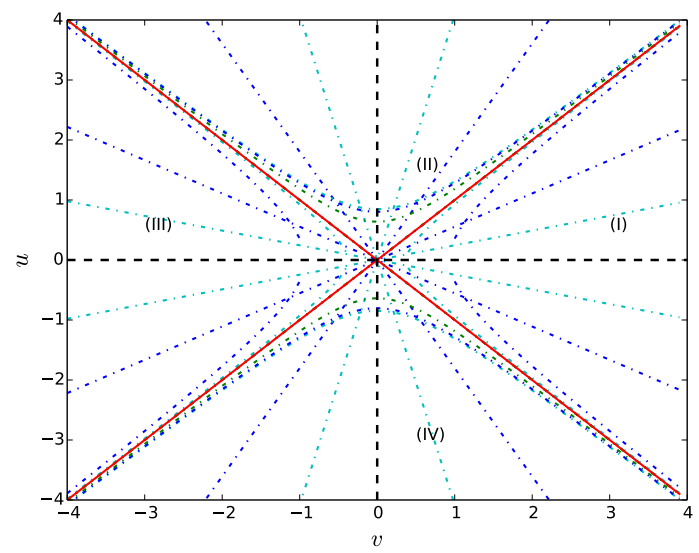

Figure 1.2: Kruskal extension of the Schwarzschild spacetime. Notice the full covering of the spacetime manifold (regions I, II, III, IV) using the Kruskal coordinates. Regions (I) and (III) represent two asymptotically flat spacetimes for $r>2 M$, which are identical. Regions II and IV, where IV is the time-reversal "version" of II, corresponds to the regions $r<2 M$ where a central singularity $r=0$ evolves. Notice that lines with constant $r$ are hyperbolae and lines of constant $t$ are straight lines going through the origin in the Kruskal geometry. The colors correspond to different parameters as follows, straight lines: $t=M$ (cyan), $t=2.5 M$ (blue), $t=10^{10} M$ (green). Hyperbolae: $r=3 M$ (blue), $r=2.5 M$ (cyan) and $r=2.1 M$ (green).

Notice that the central singularity $r=0$ gives the roots $u^{2}-v^{2}=0$. Thus in Kruskal coordinates there are two singularities, namely, $v=+\left(1+u^{2}\right)^{1 / 2}$ and $v=-\left(1+u^{2}\right)^{1 / 2}$. Moreover, notice that the asymptotically flat region $r>>2 M$ corresponds to $u^{2}>>v^{2}$. Then it can be seen that there are two asymptotically flat regions which corresponds to $u>>+|v|$ and $u<<-|v|$. Thus the Kruskal coordinates cover the whole spacetime, in contrast to the Schwarzschild coordinates which cover only the region (I) $r>2 M$. Furthermore, from (1.34) it can be seen that there is no singular behavior at $r=2 M$.

In figure 1.2 we can see that the region I correspond to the exterior Schwarzschild solution with a null hypersurface at $r=2 M$. Although the surface $r=2 M$ is not singular in the Kruskal geometry, a particle which crosses it will end up inevitably in the central singularity $r=0$. Thus region II corresponds to the 'black hole'. An interesting feature of the Kruskal coordinates is the existence of regions III and IV. For 
example, region IV is just the reverse-time version of region II. Therefore, in region IV anything goes out but nothing comes in!. This region has been denominated as a white hole $[14,66]$, a purely mathematical entity with no correspondence in reality.

\subsection{Gravitational Collapse}

As we discussed in the last section, the gravitational field in the empty exterior region $r>R$ corresponds to a patch of the Schwarzschild exterior solution. The interior of the star will be described by some energy-momentum tensor which depends on the energy density and pressure. For a homogeneous star, for example, the situation is described by the Schwarzschild interior solution [92] ${ }^{6}$. As it is expected, this interior solution must match to the exterior metric at the boundary $r=R$. The Schwarzschild radius $r=2 M$ sets the static limit for a spherically symmetric star. If the radius of the star is smaller then the Schwarzschild radius, the solution is no longer static.

In the widely accepted picture, a star supports its own weight by burning nuclear fuel which releases vast amounts of thermal and radiation pressure. Once the nuclear fuel is completely spent, the interior pressure and temperature decrease and the star contracts. For cold matter at low densities (low compared to nuclear densities which are of the order of $10^{14} \mathrm{~g} / \mathrm{cm}^{3}$ ), the pressure will be provided by quantum mechanical effects (exclusion principle). In the non-relativistic case, the pressure is estimated to be $[102]$

$$
p_{n r}=\frac{\hbar^{2}\left(3 \pi^{2}\right)^{2 / 3}}{5 m_{e}} n^{5 / 3}, \quad n<<\left(m_{e} / \hbar\right)^{3}
$$

where $n$ is the number density of electrons and $m_{e}$, is the mass of the electron. Notice that for non-relativistic matter, the degeneracy pressure is provided mainly by the electrons (baryons have bigger masses). At higher densities $\left(n>>\left(m_{e} / \hbar\right)^{3}\right)$ the situation becomes relativistic and the pressure will be given by

\footnotetext{
${ }^{6}$ We will discuss the Schwarzschild interior solution in Chapter 2.
} 


$$
p_{r}=\frac{\hbar\left(3 \pi^{2}\right)^{1 / 3}}{4} n^{4 / 3}
$$

which is independent of the mass of the fermions [32, 102]. Stars which are supported by electron degeneracy pressure are called white dwarfs. These stars have radii of about $5 \times 10^{6} \mathrm{~m}$, densities of $10^{6} \mathrm{~g} / \mathrm{cm}^{3}$ and masses of around $M_{\odot}$ [93]. As it was first shown by Stoner [97], for the case of a homogeneous mass, and generalized by Chandrasekhar [19] using a polytropic equation, the mass limit for a white dwarf is given by

$$
M_{L}=1.4\left(\frac{2}{\mu_{N}}\right)^{2} M_{\odot},
$$

where $\mu_{N}$ is the number of nucleons per electron and $M_{\odot} \approx 2 \times 10^{33} \mathrm{~g}$ is the mass of the Sun. If the density of the star is higher, the electrons become relativistic. Thus they combine with protons to produce neutrons and neutrinos (inverse beta decay). Neutrinos are radiated away, and the cold matter will be composed mainly of free neutrons. Once the star reaches a new state of equilibrium, its self-gravity will be compensated by the neutrons degeneracy pressure. This configuration is called a neutron star. Oppenheimer and Volkoff [72] studied relativistic models of neutron stars, assuming an ideal gas of free neutrons. The discovery of pulsars [43], astronomical objects which emit periodic signals, led some authors [31] to propose that these objects correspond to rotating neutron stars.

Thus, for relatively small masses, stars can be found in equilibrium states as white dwarfs or neutron stars. However, for objects with $M>M_{L}$ there are no possible nuclear or thermal reactions which can produce sufficient pressure to support the self-gravity of the star. In this "extreme" case the surface of the object will collapse behind its gravitational radius thus producing a Schwarzschild black hole.

For all practical purposes, a black hole is an invisible object. However, it has a Schwarzschild mass $M$ and its gravitational field in the region $r>2 M$ is determined 
by the Schwarzschild solution. Then, how can we detect an object which neither emits nor reflects any signal?. The strong gravitational field of the black hole will affect the dynamics of test particles in orbit dramatically. Also, accreting matter falling into the black hole will emit X-rays and radio waves which could be detected here on Earth [41].

One of the strongest candidates to contain a black hole is the system Cygnus $X-1$. This source was identified as a binary system with a high-mass star HDE 226868 and its unknown companion [17]. X-rays analysis, revealed that its companion is a super-compact object with a mass in the range of $9-15 M_{\odot}$. The accepted mass-limit for static neutron stars ${ }^{7}$ falls in the range $1.4 M_{\odot}-2.5 M_{\odot}$ thus leaving behind the hypothesis that it was a neutron star. Therefore, it is strongly believed that Cygnus $\mathrm{X}-1$ does contain a black hole.

More recently the study of Active Galactic Nuclei (AGNs), sources of powerful radiation in the center of some galaxies, has motivated some authors to suggest the existence of supermassive black holes as the originators of this phenomena. Analysis of X-rays of AGNs put black holes masses in the range $10^{6}-10^{10} M_{\odot}$. On the other hand, stellar dynamics in the central region of our galaxy (0.01 parsecs) has provided evidence of a central mass of about $2 \times 10^{6} M_{\odot}$, which is firmly believed to be a black hole. Recently [1] the LIGO interferometer has detected the first transient gravitational-wave signal which matches the waveform of an inspiral and merger of a pair of black holes with a final mass of around $62 M_{\odot}$.

\subsection{Rotating Black holes}

In section 1.2 we reviewed the Schwarzschild metric which describes the exterior gravitational field of a static spherical mass. However, it is well known that astrophysical objects (stars, planets, etc.) are rotating. Then, how is the exterior spacetime geom-

\footnotetext{
${ }^{7}$ For rapidly rotating neutron stars this limit could go up to $3.2 M_{\odot}$.
} 
etry of a rotating axisymmetric object?. Let's recall that a spacetime is considered axisymmetric if it has a Killing vector which is timelike at infinity. A particular case of this class is a static spacetime, which is characterized by being invariant under time reversal $t \rightarrow-t$.

Several authors addressed the problem of finding the metric for a rotating object (see [26, 99] for a historical review and references); however the analytical solution for the gravitational field of an axisymmetric source remained elusive for many years. In 1963 Roy Kerr [48] presented the long-sought metric for a stationary spacetime. The eminent astrophysicist S. Chandrasekhar [20] has elucidated the importance of this solution

It represents, the unique solution which the general theory of relativity provides for the description of all black holes that can occur in the astronomical universe by the gravitational collapse of stellar masses; and it is the only instance of a physical theory providing an exact description of a macroscopic object.

In Boyer-Lindquist coordinates the Kerr metric has the form [71]

$$
\begin{aligned}
d s^{2} & =-\left(1-\frac{2 M r}{\Sigma}\right) d t^{2}-\frac{2 M a r \sin ^{2} \theta}{\Sigma}(d t d \phi+d \phi d t)+\frac{\Sigma}{\Delta} d r^{2} \\
& +\Sigma d \theta^{2}+\left(r^{2}+a^{2}+\frac{2 M a^{2} r \sin ^{2} \theta}{\Sigma}\right) \sin ^{2} \theta d \phi^{2}
\end{aligned}
$$

where

$$
\Sigma(r, \theta) \equiv r^{2}+a^{2} \cos ^{2} \theta, \quad \Delta(r) \equiv r^{2}-2 M r+a^{2} .
$$

The Kerr solution is asymptotically flat and it is characterized by two constant parameters, the mass $M$ and the angular momentum $J \equiv M a$, as measured by distant observers [41]. In the non-rotating case $a=0$, the Kerr metric reduces to the 
Schwarzschild metric (1.24). Notice that the coordinates $t$ and $\phi$ does not appear explicitly in the metric elements in (1.40), thus $K=\partial_{t}$ and $\Phi=\partial_{\phi}$ are Killing vectors. This is characteristic of stationary and axisymmetric space-times [102]. The simultaneous changes in the signs, $t \rightarrow-t$, and $\phi \rightarrow-\phi$ give an isometry ${ }^{8}$. However, inversion of $t$ alone does not leave the metric invariant, except in the static case $a=0$. A time inversion produces a change in the direction of rotation.

Notice that the Kerr geometry, in contrast to the Schwarzschild metric, is well behaved at $r=0$. However, a singular behavior occurs when $r^{2}+a^{2} \cos ^{2} \theta=0$, that is, $r=0$ and $\theta=\pi / 2$. The nature of this singularity can be better understood in the Kerr-Schild coordinates $(x, y, z, \bar{t})[41]$

$$
\begin{aligned}
& x+i y=(r+i a) \sin \theta \exp \left[i \int\left(d \phi+\frac{a}{\Delta} d r\right)\right], \\
& z=r \cos \theta, \quad \bar{t}+r=\int\left(d t+\frac{r^{2}+a^{2}}{\Delta} d r\right) .
\end{aligned}
$$

In terms of $(x, y, z, \bar{t})$, the $r$-coordinate is implicitly defined by

$$
\frac{x^{2}+y^{2}}{r^{2}+a^{2}}+\frac{z^{2}}{r^{2}}=1
$$

Thus, the singularity at $r=0$ is the $\operatorname{ring} x^{2}+y^{2}=a^{2}, z=0$. Furthermore, this is a real curvature singularity as it can be shown from the invariant curvature which is given by $[105]$

$$
R_{\alpha \beta \gamma \delta} R^{\alpha \beta \gamma \delta}=\frac{48 M^{2}\left(r^{2}-a^{2} \cos ^{2} \theta\right)\left[\left(r^{2}+a^{2} \cos ^{2} \theta\right)^{2}-16 r^{2} a^{2} \cos ^{2} \theta\right]}{\left(r^{2}+a^{2} \cos ^{2} \theta\right)^{6}}
$$

which is clearly singular when $\Sigma=0$, for $M \neq 0$. On the other hand, considering a surface $r=$ constant with normal vector

\footnotetext{
${ }^{8}$ Isometries are symmetries of the metric.
} 


$$
n_{\alpha} n_{\beta} g^{\alpha \beta}=g^{r r}=\frac{\Delta}{\Sigma}
$$

we observe that the normal vector is null where $\Delta=0$ and divergent where $\Sigma=0$. Therefore $\Delta=0$ is a null hypersurface which produces the horizons

$$
r_{ \pm}=M \pm \sqrt{M^{2}-a^{2}}
$$

where $r_{+}$is called the outer horizon and $r_{-}$is the inner horizon ${ }^{9}$. The inner horizon, most likely, does not have any physical meaning, therefore, I will refer to $r_{+}$as the horizon of the Kerr black hole.

\subsubsection{THE ERGOSPHERE}

An interesting feature of the Kerr spacetime is the manifestation of stationary limit surfaces. As we discussed in the last section, for a stationary axisymmetric spacetime there are two Killing vectors, $K=\partial_{t}$ and $\Phi=\partial_{\phi}$. Thus the momenta $p_{t}$ and $p_{\phi}$, associated with $K$ and $\Phi$, are conserved quantities. The norm of $K^{\mu}$ which reads

$$
g(K, K)=g_{\mu \nu} K^{\mu} K^{\nu}=g_{t t}=-\left(1-\frac{2 M r}{r^{2}+a^{2} \cos ^{2} \theta}\right)
$$

must be negative for a standing timelike observer. However, if the right-hand side of (1.47) becomes positive then $g(K, K)$ becomes spacelike, where

$$
r^{2}-2 M r+a^{2} \cos ^{2} \theta<0
$$

Thus, if we define

$$
r_{e}^{ \pm}(M, a, \theta)=M \pm \sqrt{M^{2}-a^{2} \cos ^{2} \theta}
$$

${ }^{9}$ I will restrict only to the case $a<M$. The case $a>M$ implies a naked singularity and the extremal case $a=M$ is unstable. 
then we have the condition

$$
r_{e}^{-}(M, a, \theta)<r<r_{e}^{+}(M, a, \theta) .
$$

Therefore, any observer between the surfaces $r_{e}^{ \pm}$cannot be at rest with respect to infinity. Moreover, this observer will be dragged and will rotate in the same direction in which the central object rotates. The surfaces $r_{e}^{ \pm}$are called the stationary limit surfaces. Notice that $r_{e}^{-}$lies inside the inner horizon $r_{-}(1.46)$, so we will refer only to $r_{e}^{+}=r_{e}$. The region enclosed between the event horizon $r_{+}$and the stationary limit surface $r_{e}$ is called the ergoregion or ergosphere [20,41]. The stationary limit surface $r_{e}$ is a timelike surface with exception of the poles where it becomes null. At these two points the surfaces $r_{e}=r_{+}$coincide (see figure 1.3).

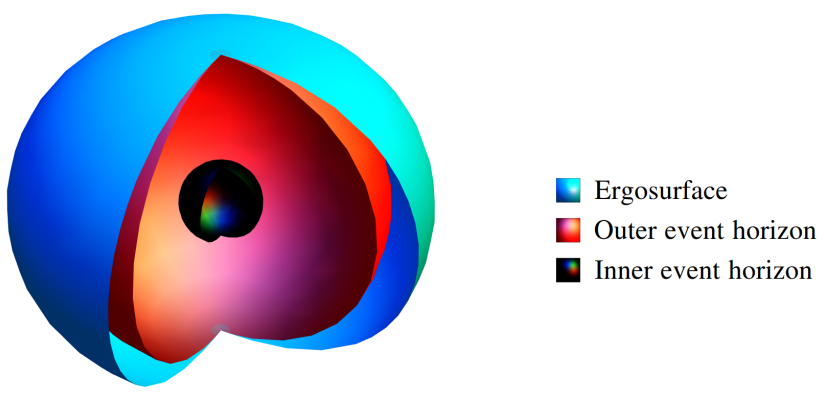

Figure 1.3: In the Kerr geometry with $0<a^{2}<m^{2}$ (in this plot $a=0.8 M$ ) the ergoregion lies between the ergosurface $r_{e}$ (cyan) and the outer event horizon $r_{+}$(red). A particle inside the ergoregion cannot be at rest with respect to infinity, but it can escape toward the region $r>r_{e}$. Particles inside the region between the horizons $r_{ \pm}$can not escape to the region $r>r_{+}$. The ring singularity $x^{2}+y^{2}=a^{2}$ lies inside the inner event horizon (black surface).

An interesting feature of the ergosurface is that timelike observers moving inside the ergoregion can escape to infinity. On the other hand, an observer moving along a timelike curve upon or inside the outer horizon $r=r_{+}$, cannot escape to the region 
$r>r_{+}$. This situation resembles the behavior of the Schwarzchild radius as a oneway surface, where anything can go in, but no signal can escape. Furthermore, notice that in the static case $a=0$, the event horizon $r_{+}$(1.46) reduces to the Schwarzschild radius $r_{s}=2 M$.

\subsubsection{What IS THE SOURCE OF THE KERR FIELD?}

From the physical point of view, it is crucial to remark that there is no Birkhoff's theorem for a rotating spacetime [105]. In contrast to a spherically symmetric and static spacetime, which Birkhoff's theorem guarantees that any solution of the vacuum field equations will be a part of the Schwarzschild spacetime, it is not true that the exterior spacetime geometry of a rotating mass is a part of the Kerr metric. The only thing that one can say is that the empty exterior spacetime geometry of a rotating object approaches asymptotically the Kerr metric. In a physical rotating object (star, planet, etc.) there are mass multipole moments (mountains, valleys, etc.) which not necessarily correspond to the multipole moments of the Kerr metric. However, multipole moments fall as $1 / r^{2+n}$, where $n$ is the nth-pole, therefore far from the source only the lowest multipole dominate. Thus, only asymptotically the Kerr geometry is relevant for a rotating body. On the other hand when a rotating mass collapses to a 'black hole,' with $|a|<M$, Robinson [88] showed that the unique stationary solution to the vacuum field equations is the family of Kerr solutions. We will review the powerful Black Hole Uniqueness Theorems in the next section.

Since its appearance in 1963 the Kerr metric has generated not only great excitement but also the question: what is the possible source for the Kerr space-time?. In contrast to the Schwarzschild space-time _ which possesses an interior solution (see section 2.3) which is matched with the exterior solution at the surface of the star _, there is no such interior (stable or unstable) solution for the Kerr field. Some authors think that this solution might not even exist [95]. Some others [99] suggest 
that the problem is irrelevant from the physical point of view, due to the fact that there is no Birkhoff's theorem for the Kerr space-time. Nevertheless, some authors have proposed models for a possible Kerr source [42, 47, 79] however; the problem remains open. We will see in chapter 4 that our results provide a long-sought solution to this problem [82].

\subsection{More ABOut Black Holes}

In 1971 Penrose and Floyd [77] showed a possible mechanism to extract energy from a rotating black hole. To understand this process let us recall that for a test particle with 4-momentum $p^{\mu}$, the conserved energy is

$$
E=-K_{\mu} p^{\mu}
$$

where $K=\partial_{t}$ and $p^{\mu}$ are timelike vectors at infinity, therefore their dot product is negative thus giving a positive energy. However one of the properties of the Kerr metric is that the Killing vector $K^{\mu}$ becomes spacelike inside the ergoregion. Consequently for a particle inside the ergosphere we have

$$
E=-K_{\mu} p^{\mu}<0
$$

In the Penrose process a particle is thrown inside the ergosphere, where it is split into two pieces $m_{1}$ and $m_{2}$. By local 4-momentum conservation we have

$$
p_{0}^{\mu}=p_{1}^{\mu}+p_{2}^{\mu}
$$

where $p_{i}^{\mu}$ correspond to the 4-momentum of the pieces. After contraction with $K_{\mu}$ and using (1.52) we have $E_{0}=E_{1}+E_{2}$. Let's arrange the situation such that $m_{1}$ falls into the black hole, therefore with negative energy $E_{1}<0$ (1.52), meanwhile $m_{2}$ escapes to infinity with energy $E_{2}$. By energy conservation we find that $m_{2}$ will come 
out with more energy than the original $E_{0}$. This extra energy is being obtained from the rotational energy of the black hole, thus reducing the mass of the black hole to $M-\left|E_{1}\right|$. Besides the energy extraction process, Penrose and Floyd observed that the surface area of the Kerr black hole

$$
A=8 \pi M r_{+}
$$

increases, even though its mass decreases. On the other hand, Christodoulou [25] showed that the irreducible mass defined as

$$
M_{i r r}^{2}=\frac{1}{2}\left[M^{2}+\left(M^{4}-J^{2}\right)^{1 / 2}\right]
$$

cannot decrease in the Penrose process, in other words, $M^{2} \geq M_{i r r}^{2}$. From (1.55) it follows that the rotational energy of the black hole is

$$
M-M_{i r r}=M-\frac{1}{\sqrt{2}}\left(M^{2}+\sqrt{M^{4}-J^{2}}\right)^{1 / 2} .
$$

For the extremal black hole $a=M, M-M_{i r r}=0.292$, which represent around $29 \%$ extracted energy of the original black hole mass-energy. Substituting $J=a M$ in (1.54) and differentiating, we obtain the first law of black hole mechanics

$$
\delta M=\frac{1}{8 \pi} \kappa \delta A+\Omega_{H} \delta J
$$

with

$$
\kappa=\frac{r_{+}-M}{2 M r_{+}}, \quad \Omega=\frac{a}{2 M r_{+}} .
$$

Here $\Omega$ corresponds to the angular velocity of the 'horizon', and $\kappa$ is the surface gravity which is defined as

$$
\kappa^{2}=-\frac{1}{2}\left(\nabla_{\mu} \xi_{\nu}\right)\left(\nabla^{\mu} \xi^{\nu}\right)
$$


where $\xi^{\nu}$ is a null Killing vector normal to some null hypersurface $\Sigma$ (Killing surface). Inspired by the particular form of (1.57), Bekenstein [8] and Smarr [94] proposed an analogy with the first law of thermodynamics

$$
d E=T d S-p d V
$$

According to Bekenstein the term $\Omega_{H} \delta J$, "represent the work done on the black hole by an external agent who increases the black hole's angular momentum. Thus $\Omega_{H} \delta J$ is the analog of $-p d V$, the work done on a thermodynamic system" [8]. On the other hand, the surface gravity $\kappa$ would be associated with the temperature of the system. Later on, Bardeen, Carter and Hawking [7] proved the first law in the stationary case. Consequently, they also proved the analog of the "zeroth law", namely that the surface gravity $\kappa$ is a constant on the horizon.

Once the analogy between black holes mechanics and thermodynamics was worked out, the next step was the study of Quantum Field Theory in curved spacetime. In 1976 Hawking [38] found that black holes are not black at all, they emit thermal radiation with a temperature

$$
T_{H}=\frac{\hbar \kappa}{2 \pi k_{B} c}
$$

where $\hbar$ is the reduced Planck constant and $k_{B}$ is Boltzmann's constant. For a Schwarzschild's black hole, where $\kappa=1 / 4 G M, T_{H}$ gives

$$
T_{s c h}=\frac{\hbar c^{3}}{8 \pi G k_{B} M} \sim 10^{-7} K\left(\frac{M_{\odot}}{M}\right)
$$

Notice that for an astrophysical black hole of $3 M_{\odot}$, the Hawking temperature is lower than the CMB (Cosmic Microwave background) temperature of $3 K$. Comparing (1.62) with the first law (1.57) the relation for entropy reads

$$
S=\frac{1}{4} \frac{A}{\hbar}
$$


which gave definitive support to Bekenstein's idea of the proportionality between entropy and the horizon area. Moreover the area theorem for classical black holes, which states that the area of the horizon never decreases in any process [37], was found sharply similar to the second law of thermodynamics. Thus the analogy between black hole mechanics and thermodynamics was finally established.

\subsubsection{Black Holes Uniqueness Theorems}

Parallel to the development of the Black Hole mechanics, some authors were interested in the geometrical properties of black holes. This development led to a series of theorems which proved that black hole spacetimes are determined uniquely by three independent parameters; mass $M$, charge $Q$ and angular momentum $J$.

Israel $[45,46]$ was the first one who showed that for any static and spherically symmetric black hole, the external gravitational field is determined uniquely by two parameters; its mass $M$ and charge $Q$. Furthermore, these fields are described by the Schwarzschild solution, when $Q=0$, and the Reissner-Nordstrom family of solutions with $M \geq G^{1 / 2}|e| / c$.

As an extension of Israel's results, Carter [15] showed that black hole exterior solutions, which are axisymmetric, are determined uniquely, at least, by two independent parameters; the mass $M$ and angular momentum $J$. Moreover, he showed that the Kerr metric is the only one admitting zero angular momentum. Robinson [88] gave the definitive proof that the Kerr metric, with $|a|<M$, is the unique stationary black hole solution to the Einstein vacuum field equations.

Finally, the uniqueness theorem for the Kerr-Newmann [69] rotating charged black hole, was nailed down by Mazur [58] who showed that general black holes are characterized uniquely by three parameters; mass $M$, charge $Q$ and angular momentum $J$. Moreover, the gravitational field of a stationary and charged black hole is determined only by the Kerr-Newman solution to the Einstein-Maxwell system, under the 
constraint $M^{2}-a^{2}-Q^{2}>0$.

The implications of these theorems are dramatic. For instance, an asymmetric object which suffers gravitational collapse, would radiate away all its multipole moments leaving only its mass $M$, charge $Q$ and angular momentum $J$. Following the aphorism introduced by Ruffini and Wheeler [89], "a black hole has no hair".

\subsection{Paradoxes AND UnSOLVEd ISSUES}

Despite the developments discussed in the previous sections, in addition to the vast amount of literature on the subject (see e.g. $[73,103]$ and references therein), the physical reality of black holes has not only generated some skepticism [5, 29, 40] but also has raised some paradoxical issues which have not been consistently solved. Below I will discuss some of these issues.

- Although the analytic continuation (see section 1.2) is commonly accepted as a valid mathematical procedure to extend the Schwarzschild spacetime through the null hypersurface $r_{S}=2 M$, it involves the assumption that the energymomentum tensor $T_{\mu \nu}$ vanishes there. However, the hyperbolic character of Einstein's equations allows for sources which do not satisfy this assumption $[67,60]$.

- Besides the non-conservation of information by quantum matter falling into a black hole [39], in the original Hawking radiation derivation [38] the backwardin-time propagated mode

$$
\hbar \omega \sim \frac{k_{B} T_{H}}{\left(1-\frac{2 M}{r}\right)^{1 / 2}}
$$

seems to experience an arbitrary large blueshift near the horizon $r_{S}=2 M$ with energies greater than the Planck energy. It is expected that these highly 
'blue-shifted' photons would leave a non-negligible 'imprint' on the spacetime geometry, making the approximation of fixed classical geometry background untenable $[60,67]$.

- The fact that the temperature of a black hole is inversely proportional to its mass (1.62), implies that its heat capacity

$$
\frac{d E}{d T_{H}}=-\frac{8 \pi G k_{B} M^{2}}{\hbar c},
$$

is negative. However, it is well known from statistical mechanics that the heat capacity, for a system in equilibrium, is proportional to the energy fluctuations $[59]$

$$
c_{V}=\left(\frac{d\langle E\rangle}{d T}\right)_{V}=\frac{1}{k_{B} T^{2}}\left\langle(E-\langle E\rangle)^{2}\right\rangle,
$$

which is clearly positive.

- The differential form of the first law of black hole mechanics (1.57) might suggest that

$$
\left(\frac{\partial M}{\partial A}\right)_{J}=\frac{\kappa}{8 \pi}
$$

could be associated to the surface tension of the event horizon [25]. However, if we analytically extend the black hole solution through the surface $r_{S}=2 M$ (see section 1.2) it is assumed that the energy-momentum tensor vanishes there. Thus it is a mystery to what kind of surface energy it is possible to associate the surface gravity (1.67).

- Is well known from thermodynamics that a cold system has associated a low entropy. The Bekenstein-Hawking formula (1.62), predicts that in the limit 
when $\hbar \rightarrow 0$ (or $M \rightarrow \infty)$ the black hole temperature goes to zero. However the entropy of a black hole, according to Bekenstein [8], is given by

$$
S_{B H}=\gamma \frac{16 \pi G k_{B}}{\hbar c} M_{i r r}^{2}
$$

which goes to infinity when $\hbar \rightarrow 0$. Thus, in principle, a black hole has an infinitely large entropy at zero temperature! [67].

It is believed that the resolution of these paradoxes will be achieved in the framework of a quantum theory of gravity. Despite considerable efforts in the last decades (see [106] for a review and references therein), we still do not possess such a theory. Therefore it is imperative to investigate alternative solutions to the inconsistencies of black holes. Some models have been introduced to alleviate some of the black hole paradoxes $[10,22,96]$. In particular, we concentrate in the gravastar (vacuum condensate gravitational star) model proposed by Mazur and Mottola [60, 61, 62]. We will review this model in the next chapter. 


\title{
CHAPTER 2
}

\section{Gravitational Vacuum Condensate Stars}

\author{
You tremble, carcass? You would \\ tremble even more if you knew \\ where I am going to take you
}

Vicomte de Turenne

\section{INTRODUCTION}

As we discussed in section 1.3 , it is commonly accepted that the final state of complete gravitational collapse is a singular state called a black hole $[41,66,102]$. However, in section 1.6, we discussed certain paradoxical behaviors associated with black holes which have not been consistently solved. This situation, in addition to the lack of definitive observational proof of the existence of the event horizon [5], has originated some skepticism in the community regarding the physical reality of black holes $[29,40]$.

Some alternatives to black holes have been proposed [22, 27, 30, 53]. In particular, we concentrate on Gravastars (Gravitational Vacuum Condensate Stars) proposed by Mazur and Mottola [60,61, 62, 63]. A gravastar is the outcome of the gravitational collapse of a star, to the Schwarzschild radius $R_{s}$. Essentially a gravastar consists of an interior region of negative pressure $p=-\epsilon$, composed of low-temperature weakly interacting bosons. This interior region is described by a modified de Sitter spacetime, with a boundary consisting of a thin shell of ultrarelativistic fluid with equation of state $p=\epsilon$. The latter is matched to the spherically symmetric Schwarzschild exterior 
solution in the vacuum.

In the gravastar model, the spacetime suffers a quantum phase transition, which leads to $p=-\epsilon$, starting at the center of the star and moving outwards thus releasing huge amounts of energy and entropy [63]. As suggested by Gliner [30] the idea of an interior vacuumlike state (dark energy), jointly with a quantum phase transition which transforms matter into that state, is probably the only conceivable alternative to avoid the manifestation of a singularity. ${ }^{1}$

As it is well known the source of gravitational fields, in the Einstein equations, is the trace of the energy-momentum tensor, $3 p+\epsilon$. In the vacuumlike energy case, $p=-\epsilon$, gravity becomes repulsive thus originating divergence of geodesics. Moreover, the EOS for vacuum energy violates the strong energy condition $\epsilon+3 p \geq 0$. As it was first suggested by Gliner [30], during gravitational collapse a phase transition will occur such that the collapsing object becomes a vacuumlike state. Therefore its gravitational repulsion would avoid the formation of a singular state.

This chapter is organized as follows: in section 2.1 we review the original gravastar model proposed by Mazur \& Mottola [60, 61, 62]. In connection with gravastars, in section 2.2 we discuss the interior solution found by Schwarzschild [92] for a homogeneous spherical mass of constant energy density. This solution is characterized by the appearance of a divergence in pressure when the radius of the star $R=(9 / 8) R_{S}$, known as the Schwarzschild-Buchdahl bound. In section 2.3 we analyze the manifestation of a negative pressure in the Schwarzschild interior solution when $R<(9 / 8) R_{S}$. Using the Komar formula [50], we show that the divergence in pressure is integrable producing a transverse pressure and a surface tension on a surface of discontinuous pressure. One result of particular importance is that, in the ultracompact limit $R=R_{S}$, the Schwarzschild interior solution becomes a gravitational condensate with

\footnotetext{
${ }^{1}$ The equation of state $p=-\epsilon$ is associated with the vacuum energy or cosmological constant $\Lambda$, which is believed to be the cause of the accelerated cosmological expansion [78, 87].
} 
a modified de Sitter interior with negative pressure $p=-\epsilon$ and a finite surface tension [63]. Thus, essentially in the ultra compact limit $R=R_{S}$ the Schwarzschild star becomes the gravastar.

\subsection{Gravitational vacuUmlike COndensate model}

The starting point is a spherically symmetric spacetime in Schwarzschild coordinates

$$
d s^{2}=-e^{2 \nu(r)} d t^{2}+e^{2 \lambda(r)} d r^{2}+r^{2}\left(d \theta^{2}+\sin ^{2} \theta d \phi^{2}\right)
$$

The stress-energy tensor for a spherically symmetric perfect fluid is given by (see section 1.1)

$$
T_{\nu}^{\mu}=\left(\begin{array}{cccc}
-\epsilon & 0 & 0 & 0 \\
0 & p & 0 & 0 \\
0 & 0 & p_{\perp} & 0 \\
0 & 0 & 0 & p_{\perp}
\end{array}\right)
$$

where $\epsilon, p$ and $p_{\perp}$ correspond to the energy density, radial pressure, and tangential pressure respectively, which are functions of $r$ only. In general the radial pressure $p=T_{r}^{r}$ is different from the tangential pressure $p_{\perp}=T_{\theta}^{\theta}=T_{\phi}^{\phi}$. However, to discuss the simplest case, we assume $p=p_{\perp}$. The energy density $\epsilon$ and the pressure $p$ are related through a given one-parameter EOS. The relevant components of the Einstein equation $G^{\mu}{ }_{\nu}=8 \pi T^{\mu}{ }_{\nu}$ are

$$
\begin{aligned}
& e^{-2 \lambda}\left(2 r \frac{d \lambda}{d r}-1+e^{2 \lambda}\right)=8 \pi \epsilon r^{2}, \\
& e^{-2 \lambda}\left(2 r \frac{d \nu}{d r}+1-e^{2 \lambda}\right)=8 \pi p r^{2},
\end{aligned}
$$

jointly with the energy-momentum conservation relation 


$$
\nabla_{\mu} T_{r}^{\mu}=\frac{d p}{d r}+(\epsilon+p) \frac{d \nu}{d r}+\frac{2}{r}\left(p-p_{\perp}\right)=0
$$

which corresponds to the relativistic generalization of the hydrostatic equilibrium equation or Tolman-Oppenheimer-Volkoff (TOV) equation. In its original version $[60,62]$ the gravastar model is presented as a patch of de Sitter spacetime, with an EOS $p=-\epsilon$, which fills the interior of the compact object. The exterior region consists of a thin-shell of ultra-stiff fluid with $\operatorname{EOS~} p=\epsilon$ which is matched to a spherically symmetric Schwarzschild vacuum spacetime $(p=\epsilon=0)$. Additionally, this thin shell gravastar requires two infinitesimal thin shells, with surface densities $\sigma_{ \pm}$and surface tensions $\varrho_{ \pm}$, which stabilize the construction. In summary

$$
\begin{cases}\text { I. } \quad p=-\epsilon, \quad 0 \leq r<R_{i}, & \text { (interior) } \\ \text { II. } p=+\epsilon, \quad R_{i}<r<R, & \text { (thin shell) } \\ \text { III. } p=\epsilon=0, \quad R<r, & \text { (exterior) }\end{cases}
$$

where $R_{i}$ and $R$ denote the interior and exterior interfaces of region II. Following this description, the non-singular interior region is described by a section of a de Sitter spacetime [41], given by the metric function

$$
f(r) \equiv e^{2 \nu(r)}=C\left(1-H^{2} r^{2}\right), \quad O \leq r \leq R_{i}
$$

where $C$ is a constant which will be determined later, and $H$ is defined by

$$
\epsilon \equiv \frac{3 H^{2}}{8 \pi}
$$

The exterior, asymptotically flat, region corresponds to the spherically symmetric and empty Schwarzschild space-time (1.24) with metric function

$$
f(r)=h(r)=1-\frac{2 M}{r}, \quad R<r
$$


where we have introduced the notation $h(r) \equiv e^{-2 \lambda(r)}$. In order to discuss the infinitesimal shell region, let's define the parameter $w \equiv 8 \pi r^{2} p$. Thus the equation (2.3) with $\operatorname{EOS~} p=\epsilon$ gives

$$
\frac{d}{d r}[r(1-h)]=w
$$

which reduces to

$$
\frac{d r}{r}=\frac{d h}{1-w-h}
$$

In terms of $w$, the TOV equation (2.5) reads

$$
\frac{1}{r^{2}} \frac{d w}{d r}-\frac{2 w}{r^{3}}=-\frac{w}{f r^{2}} \frac{d f}{d r}
$$

Combining (2.12) with (2.4), and after some algebra, we find

$$
\frac{d h}{h}=-\left(\frac{1-w-h}{1+w-3 h}\right) \frac{d w}{w} .
$$

In general, equations (2.11) and (2.12) must be integrated numerically. However in the approximation of a very thin shell, $R_{i} \approx R$ and $0<h<<1$, equation (2.13) can be integrated analytically (at leading order in $h$ ) to give

$$
h \approx \varsigma \frac{(1+w)^{2}}{w}<<1, \quad \varsigma=\text { const. }
$$

From (2.11) and (2.12) we have

$$
\frac{d r}{r}=-\frac{h}{1+w-3 h} \frac{d w}{w}
$$

Substituting (2.14) into (2.17) we have

$$
\frac{d r}{r}=-\varsigma \frac{(1+w)}{w^{2}} d w
$$


Inside the thin shell $d r$ is of the order of $\varsigma d w$, thus the change in the $r$ coordinate is very small. In the same region the metric function $f(r)$, which satisfies the condition $\epsilon r^{2} f=w f=$ const., is found to be

$$
f(r) \approx \frac{w_{i}}{w} f\left(R_{i}\right) .
$$

By continuity the induced three-metric functions $f, h$ must be continuous at the interfaces $r=R_{i}$ and $r=R$. However, the first derivatives of $f, h, p$ will be discontinuous by consequence of the Einstein equations components (2.3), (2.4) and (2.5).

We can take advantage of the continuity of the metric functions at the interfaces, in order to find explicit expressions for the constants $C, H, M$. At the interface $R_{i}$; $f^{\mathrm{I}}\left(R_{i}\right)=f^{\mathrm{II}}\left(R_{i}\right), h^{\mathrm{I}}\left(R_{i}\right)=h^{\mathrm{II}}\left(R_{i}\right)$, gives the relations

$$
\begin{aligned}
& C\left(1-H_{0}^{2} R_{i}^{2}\right)=f\left(R_{i}\right), \\
& 1-H^{2} R_{i}^{2}=\varsigma \frac{\left(1+w_{i}^{2}\right)}{w_{i}} .
\end{aligned}
$$

Solving for $H$ from (2.19) we have

$$
H^{2}=\frac{1}{R_{i}^{2}}\left[1-\varsigma \frac{\left(1+w_{i}^{2}\right)}{w_{i}}\right] .
$$

Similarly, at the interface $r=R, h^{\mathrm{II}}(R)=h^{\mathrm{III}}(R)$ gives

$$
\varsigma \frac{\left(1+w_{R}^{2}\right)}{w_{R}}=1-\frac{2 M}{R} .
$$

Solving for M, (2.21) gives

$$
M \equiv m(R)=\frac{R}{2}\left[1-\varsigma \frac{\left(1+w_{R}^{2}\right)}{w_{R}}\right] .
$$

The constant $C$ can be found as follows: at the interface $r=R$ we have 


$$
\frac{f^{\mathrm{III}}(R)}{h^{\mathrm{III}}(R)}=\frac{f^{\mathrm{II}}(R)}{h^{\mathrm{II}}(R)}=1 .
$$

Using the relations (2.14) and (2.17) for the region II, we have

$$
\frac{f^{\mathrm{II}}(R)}{h^{\mathrm{II}}(R)}=\frac{w_{i} f\left(R_{i}\right)}{\varsigma\left(1+w_{R}^{2}\right)}=1 .
$$

Substituting (2.18) and (2.18) into (2.24) we obtain finally

$$
C=\left(\frac{1+w_{R}}{1+w_{i}}\right)^{2}
$$

One caveat that one might rise, is that the energy density $\epsilon(r)$ and the pressure $p(r)$ are discontinuous by virtue of the Einstein equations (2.3), (2.4). From the TOV equation (2.5), it can be shown that these discontinuities can be avoided if there is an infinitesimal thin-shell with anisotropic pressures $p_{\perp} \neq p[16]$. One of the advantages of these anisotropic pressures, is their continuity, although we still need to specify an EOS. An example of this will be given in the next section where we discuss the Schwarzschild interior solution and its connection with gravastars.

\subsection{SCHWARZSCHILD INTERIOR SOLUTION}

In this section we review the Schwarzschild interior solution [92] which describes the interior geometry of a spherical configuration of uniform energy density ${ }^{2}$

$$
\epsilon=\epsilon_{0}=\text { constant }, \text { for all } p \text {. }
$$

Although a realistic star might be described by a more complicated equation of state, nevertheless the Schwarzschild interior solution provides an interesting and instructive limiting model. The uniform density approximation might lead to the misconception

\footnotetext{
${ }^{2}$ The Schwarzschild interior solution is discussed in standard general relativity textbooks (see e.g. $[14,80,95,102])$
} 
of "incompressible fluid" which would imply a limitless speed of sound $v=(d p / d \rho)^{1 / 2}$ in contradiction with one of the pillars of special relativity, the principle of causality. One can imagine a fluid having a composition which varies point to point, such that the density in the region of high pressure is the same as the density in the low-pressure region $[66]$.

We follow the discussion given by the author [82]. The relevant equations for the situation have been written above (2.1)-(2.5). In addition to those, it is conventional to introduce

$$
h(r)=1-\frac{2 m(r)}{r},
$$

where the function $m(r)$ is associated with the mass within a radius $r$ and is given by the Misner-Sharp relation [66]

$$
m(r)=\int_{0}^{r} d r 4 \pi r^{2} \epsilon
$$

In terms of $(2.28),(2.4)$ becomes

$$
\frac{d \nu}{d r}=\frac{m(r)+4 \pi p r^{3}}{r[r-2 m(r)]},
$$

which in the non-relativistic limit reduces to Poisson's equation $d \nu / d r=m(r) / r^{2}$, where $\nu(r)$ is associated to the Newtonian gravitational potential. The interior solution, or Schwarzschild star, is matched at the boundary $r=R$ to the asymptotically flat vacuum exterior Schwarzschild solution

$$
e^{2 \nu(r)_{e x t}}=h_{e x t}(r)=1-\frac{2 M}{r}, \quad r \geq R
$$

where $M$ is the total mass and $R$ is the radius of the star. The functions $p(r)$, determined by the TOV equation (2.5), and $m(r)$ given by (2.28), satisfy the boundary conditions $p(R)=0$ and $m(R)=M$. The interior of the star is modeled as an incompressible and isotropic fluid $p=p_{\perp}$ with 


$$
\epsilon=\bar{\epsilon}=\frac{3 M}{4 \pi R^{3}}=\text { const. }
$$

It is useful to define [63]

$$
H^{2}=\frac{R_{s}}{R^{3}}
$$

where $R_{S}=2 M$ is the Schwarzschild radius. In terms of (2.32), equations (2.27) and (2.28) can be solved to obtain

$$
m(r)=\frac{4 \pi}{3} \bar{\epsilon} r^{3}=M\left(\frac{r}{R}\right)^{3}, \quad h(r)=1-H^{2} r^{2}, \quad 0 \leq r \leq R .
$$

From (2.5) the pressure takes the form

$$
p(r)=\bar{\epsilon}\left[\frac{\sqrt{1-H^{2} r^{2}}-\sqrt{1-H^{2} R^{2}}}{3 \sqrt{1-H^{2} R^{2}}-\sqrt{1-H^{2} r^{2}}}\right] .
$$

In figure 2.1 is plotted the pressure $p$ (in units of the density $\epsilon$ ) as a function of the radial distance $r$ (in units of the radius of the star $R$ ) for the Schwarzschild interior solution, for several values of the compactness parameter $R / R_{S}$. The metric function $e^{2 \nu(r)}$ for $r<R$ can be computed to give

$$
f(r) \equiv e^{2 \nu(r)}=\frac{1}{4}\left[3 \sqrt{1-H^{2} R^{2}}-\sqrt{1-H^{2} r^{2}}\right]^{2} \geq 0 .
$$

Across the boundary of the configuration $r=R$, this function must match the exterior metric (1.24). The continuity of $f(r)$ guarantees that an observer crossing the boundary will not notice any discontinuity of time measurements. In figure 2.2 the redshift factor $\sqrt{f}$ is plotted against $r / R$ for several values of $R>(9 / 8) R_{S}$. Notice that $(2.35)$ is regular except at some radius $R_{0}$ where the denominator in $(2.34)$

$$
D \equiv 3 \sqrt{1-H^{2} R^{2}}-\sqrt{1-H^{2} r^{2}}
$$




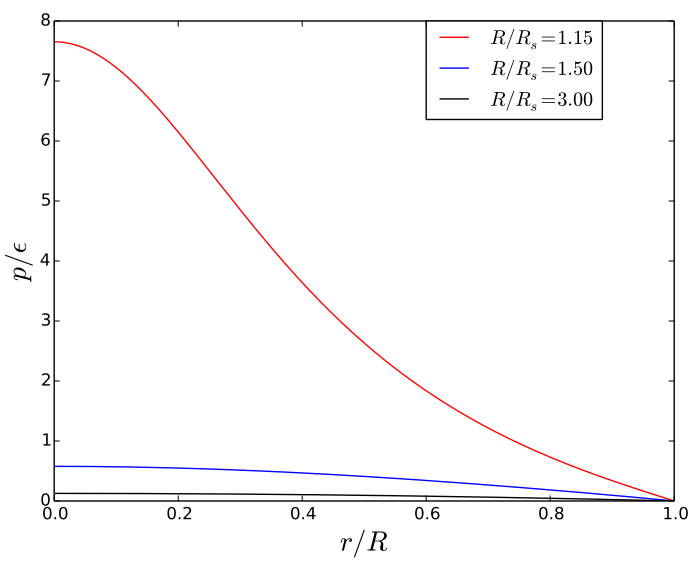

Figure 2.1: Pressure (in units of $\epsilon$ ) as a function of $\mathrm{r}$ (in units of the radius $R$ ) for the Schwarzschild star for several values of the compactness parameter $R / R_{S}$ above the Schwarzschild-Buchdahl bound. Notice how the pressure rapidly increases when the radius of the star approaches the Schwarzschild-Buchdahl bound $R_{B}=(9 / 8) R_{S}$.

vanishes in the range $0<r<R$. Remarkably, it can be seen from (2.34) and (2.35) that the pressure goes to infinity at the same point where $f(r)=0$. This singular radius can be found directly from $(2.36)$ to be

$$
R_{0}=3 R \sqrt{1-\frac{8}{9} \frac{R}{R_{s}}},
$$

which is imaginary for $R / R_{S}>9 / 8$. In this regime, $p(r)$ and $f(r)$ are positive. Moreover, when $R \rightarrow(9 / 8) R_{S}^{+}$from above, $(2.37)$ shows that $R_{0}$ approaches the real axis at $R_{0}=0$ and a divergence of the pressure appears jointly with $f(r) \rightarrow 0$. This limit value $R_{B}=(9 / 8) R_{S}$, or Schwarzschild-Buchdahl bound [13, 92], fixes the maximum possible mass for a star with given radius $R$. At this radius $R_{B}$ general relativity predicts that the star cannot remain in static equilibrium. Furthermore, once the star reaches this critical point, its gravitational collapse is inevitable. 


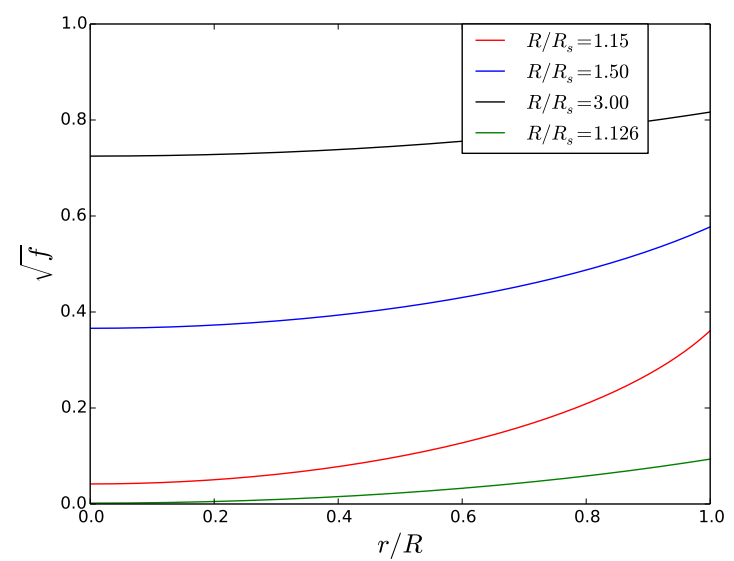

Figure 2.2: Redshift factor $\sqrt{f}$ as a function of $r$ (in units of $R$ ) for the Schwarzschild star for several values of the compactness parameter $R / R_{S}$ above the Schwarzschild-Buchdahl bound. Notice how $\sqrt{f}$ approaches zero when the radius of the star approaches the Schwarzschild-Buchdahl bound $R_{B}=(9 / 8) R_{S}$.

\subsection{SCHWARZSCHILD STAR IN THE 'BLACK HOLE' LIMIT}

Due to the manifestation of a divergence in pressure at the Schwarzschild-Buchdahl bound, in addition to the incompressible fluid approximation being considered artificial [68], the Schwarzschild star solution for $R<(9 / 8) R_{S}$ has been long-time ignored in the literature [14, 68, 80, 102]. Recently Mazur \& Mottola [63] analyzed the Schwarzschild star in the region $R_{s}<R<(9 / 8) R_{s}$ and they found that the zero of $D$ given by (2.37) moves outwards from the origin to finite values $0<R_{0}<R$ (see figure 1.2). Then there emerges a region where $p(r)<0, f(r)>0$ and $D<0$, covering the range $0 \leq r<R_{0}$. As the radius of the star keeps approaching the Schwarzschild radius from above $R \rightarrow R_{s}^{+}, R_{0} \rightarrow R_{s}^{-}$from below, where $R_{0}$ is given by (2.37) which corresponds to the radius of the sphere where the pressure is divergent and $f\left(R_{0}\right)=0$. Analysis of (2.34) shows that the new interior region becomes one of constant negative pressure $p=-\epsilon$ for $r<R=R_{0}=R_{s}$ (see figure 2.4). In this limit, the interior metric function (2.35) becomes 


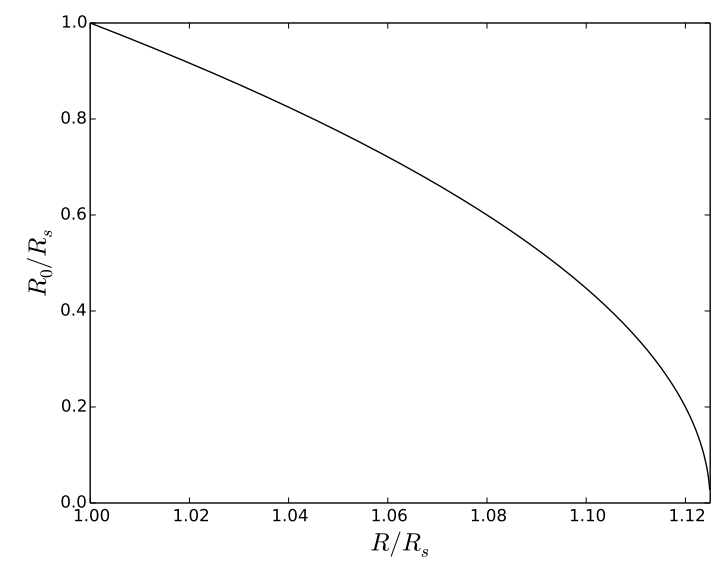

Figure 2.3: $R_{0}$ as a function of $R$ (in units of $R_{s}$ ).

$$
f(r)=\frac{1}{4}\left(1-H^{2} r^{2}\right)=\frac{1}{4} h(r)=\frac{1}{4}\left(1-\frac{r^{2}}{R_{s}^{2}}\right), \quad H=\frac{1}{R_{s}}
$$

which is a patch of de Sitter spacetime [41], but modified by a factor of (1/4) in the $g_{t t}$ component. This factor, which was undetermined in [62] (see section 2.1), is key for the correct matching of the interior de Sitter to the exterior region $r>R_{s}$ which remains the vacuum spherically symmetric Schwarzschild geometry (1.24). Instead of an 'event horizon', there is an infinitesimal thin shell discontinuity at $R_{s}=2 M$ where there is a jump in pressure and the zeroes $f=h=0$ of the interior modified de Sitter and exterior vacuum Schwarzschild spacetimes match.

Although a gravastar does not have event horizon, $R=R_{s}$ is a null hypersurface. However, in contrast to the black hole, the gravastar does not require the interior region $r<R_{s}$ to be trapped ${ }^{3}$. Moreover, the gravastar solution with interior $p=-\epsilon$, has no entropy and zero temperature, thus validating its condensate state nature.

Mazur \& Mottola [63] showed that the divergence in pressure at $R_{0}$ can be studied through the Komar integral $[50,81]$. The motivation to introduce this formalism ${ }^{4}$, is to construct covariant conservation laws in general relativity.

\footnotetext{
${ }^{3}$ For a definition of trapped surface see [102].

${ }^{4}$ See Appendix A for a brief review of the Komar integral.
} 


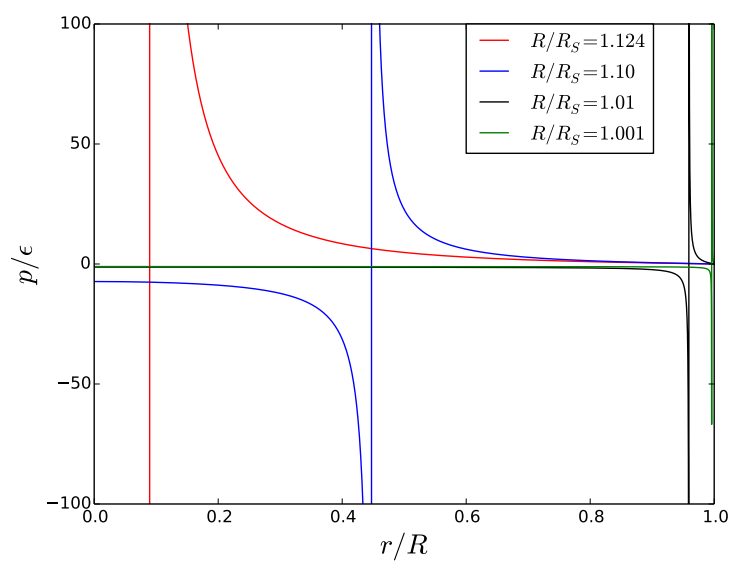

Figure 2.4: Pressure (in units of $\epsilon$ ) as a function of $\mathrm{r}$ (in units of the radius of the star $R$ ) of the interior Schwarzschild solution for various values of the ratio $R / R_{s}$ below the Buchdahl bound. Notice the approach of the negative interior pressure $p \rightarrow-\epsilon$ as $R \rightarrow R_{s}^{+}$ from above and $R_{0} \rightarrow R_{s}^{-}$from below.

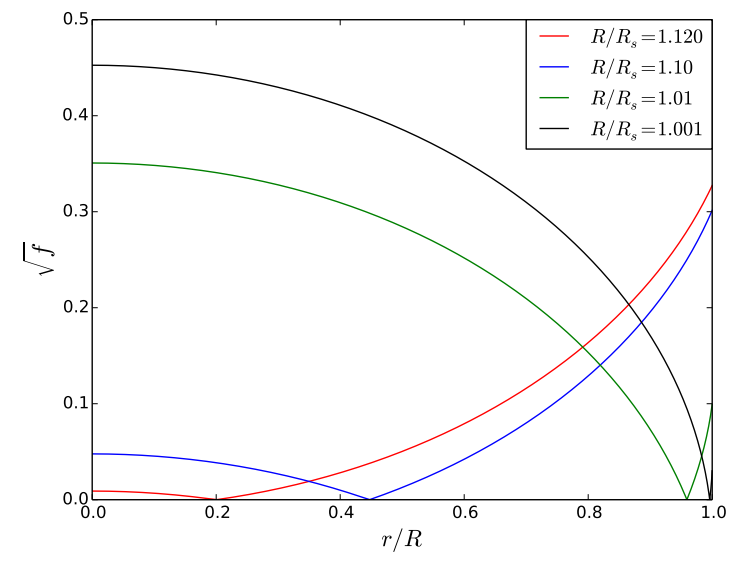

Figure 2.5: Redshift factor as a function of $\mathrm{r}$ (in units of the radius $R$ ) of the interior Schwarzschild solution for various values of $R<1.125 R_{s}$. Note how $R_{0}$ (the zero of $\sqrt{f}$ ) starts moving from inside approaching the radius of the star $R$. Meanwhile the radius of the star $R$ approaches the Schwarzschild radius from above.

\subsubsection{CONSERVED MASS AND SURFACE GRAVITY}

Using equations (A.6) and (A.9) we have the following relation

$$
\int_{\partial \Sigma} d \Sigma_{\mu \nu} \nabla^{\mu} K^{\nu}=4 \pi \int_{\Sigma}\left(2 T_{\nu}^{\mu}-T \delta_{\nu}^{\mu}\right) K^{\nu} d \Sigma_{\mu}
$$

where $d \Sigma_{\mu}=e_{\mu}^{0} d^{3} x \sqrt{\gamma}$, with $d V=d^{3} x \sqrt{\gamma}$ the volume element of the induced metric 


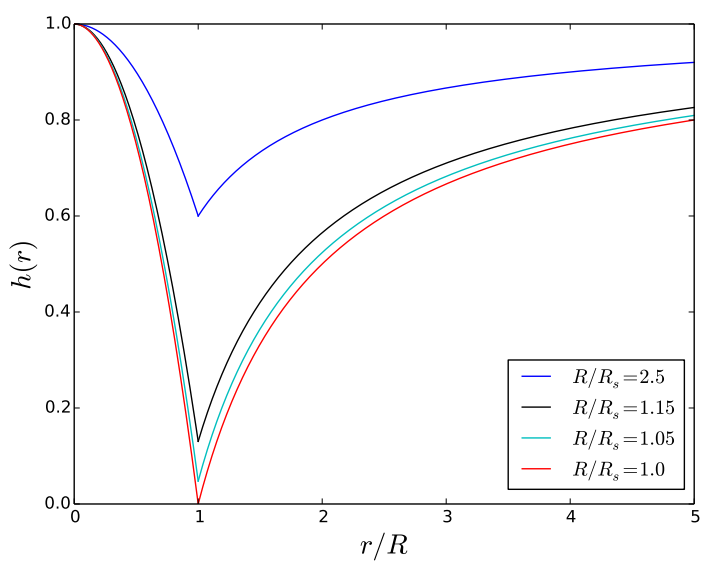

Figure 2.6: Metric function $h(r)$ as a function of $r / R$ of the interior and exterior Schwarzschild solution (gravastar spacetime). Note how the minimum of $h(r)$ approaches zero when the radius of the star $R$ approaches the Schwarzschild radius from above.

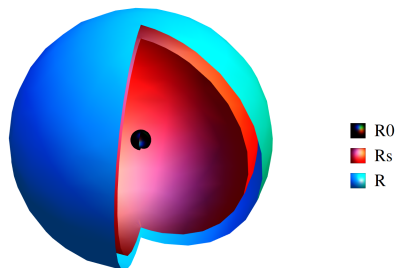

(a) $R / R_{s}=1.124$

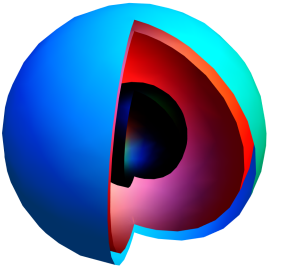

- $\mathrm{R} 0$
Rs
- $\mathrm{R}$

(b) $R / R_{s}=1.10$

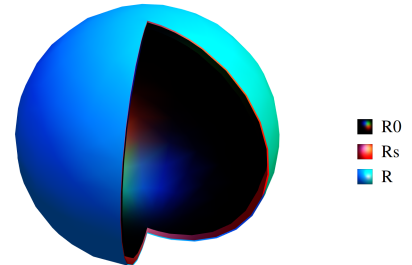

(c) $R / R_{s}=1.0125$

Figure 2.7: Pictorial diagram of the Schwarzschild star in the regime $R_{s}<R<(9 / 8) R_{s}$, showing the approach of the surface of the star $R$ (cyan) to the Schwarzschild surface $R_{s}$ (red). The radius of the star is measured in units of the Schwarzschild radius $R_{s}$. The surface $R_{0}$ (black) where the pressure diverges (and $f=h=0$ ) is shown at different stages. Figure $2.7 \mathrm{a}$, shows that $R_{0}$ emerges at the center of the star where the fluid suffers a phase transition. The region $0 \leq r<R_{0}$ with negative pressure starts approaching $R_{s}$ from below, meanwhile the radius of the star $R$ approaches $R_{s}$ from above (see figure. 2.7b). In the gravastar limit when $R \rightarrow R_{s}^{+}$and $R_{0} \rightarrow R_{s}^{-}$, the whole interior region is one of constant negative pressure given by a static patch of modified de Sitter spacetime with a finite surface tension (see figure 2.7c). The exterior spacetime is described by the standard vacuum Schwarzschild metric. Instead of an event horizon, an infinitely thin shell forms at the Schwarzschild radius $R_{s}$ where there is a jump in pressure and the zeroes $f=h=0$ of the interior modified de Sitter and exterior Schwarzschild solutions match (Figure adapted from the author's paper [82]). 


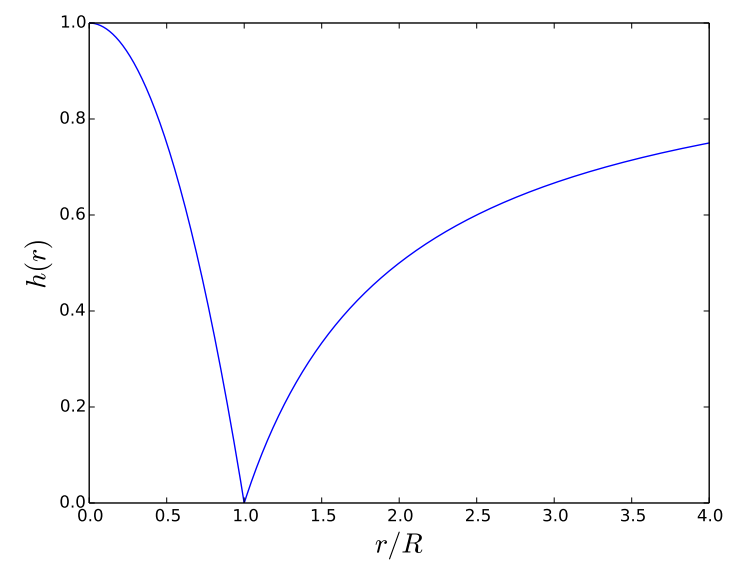

Figure 2.8: Metric function $h(r)$ as a function of $r$ (in units of the radius $R$ ) for a gravastar where $R=R_{S}$. Note in particular the non-analytic cusp behavior at $r=R=R_{S}$.

$\gamma_{i j}$ and $e_{\mu}^{0}$ the vierbein which satisfies [14]

$$
g_{\mu \nu} e_{a}^{\mu} e_{b}^{\nu}=\eta_{a b}
$$

where $\eta_{a b}$ is the Lorentzian metric. If we consider a three-volume encompassed by an outer two-surface $\partial V_{+}$and an inner two-surface $\partial V_{-}$, equation (2.39) gives (see [63] for details)

$$
\frac{1}{4 \pi} \int_{\partial V_{+}} \kappa_{f} d A-\frac{1}{4 \pi} \int_{\partial V_{-}} \kappa_{f} d A=\int_{V}\left(2 T_{\alpha}^{\mu}-T \delta_{\alpha}^{\mu}\right) K^{\alpha} u_{\mu} d V
$$

where $u_{\mu}$ is the 4 -velocity of a particle at rest, respect to $t, \kappa_{f}=\frac{1}{2} e_{\nu}^{1} f^{-1 / 2} \nabla^{\nu} f$ is the surface gravity which is written as a function of $f=-g_{t t}=-K_{\mu} K^{\mu}$. Comparing (2.41) with (A.9) (with $E=M$ ) we have

$$
M=\int_{V}\left(2 T_{\alpha}^{\mu}-T \delta_{\alpha}^{\mu}\right) K^{\alpha} u_{\mu} d V+\frac{1}{4 \pi} \int_{\partial V_{-}} \kappa_{f} d A
$$

Thus, the total energy of the system corresponds to a term associated with the volume integral of $T^{\mu}{ }_{\nu}$ and a possible contribution from the inner surface $\partial V_{-}$. As we discussed in chapter 1 the association of a surface gravity to the classical black hole horizon is problematic (see section 1.6). We will see that the meaning of a 
surface gravity only makes sense when we consider a regular interior solution, as the Schwarzschild star. It is the difference in surface gravities which provides a physical surface tension, in complete contrast with the standard analytic continuation in classical black holes where it is not clear to what source the surface tension is associated to.

Using the Einstein equations (2.3) and (2.4) with a source given by (2.2), and applying (2.42) we obtain

$$
\frac{d}{d r}\left[r^{2} \kappa(r)\right]=4 \pi \sqrt{\frac{f}{h}} r^{2}\left(\epsilon+p+2 p_{\perp}\right) .
$$

If we substitute the Schwarzschild interior solution, with $p_{\perp}=p$, and the metric function $\sqrt{f(r)}=\frac{1}{2} D$, where $D$ is given by (2.36), into the RHS of (2.43) we have

$$
4 \pi \sqrt{\frac{f}{h}} r^{2}(\epsilon+3 p)=4 \pi r^{2} \bar{\epsilon} \sqrt{\frac{f}{h}}\left[\frac{\sqrt{1-H^{2} r^{2}}}{D}\right]=4 \pi r^{2} \bar{\epsilon} \operatorname{sgn}(D), \quad r \neq R_{0}
$$

where the signum function satisfies

$$
\operatorname{sgn}(D)=\operatorname{sgn}\left(r-R_{0}\right) \begin{cases}=-1, & r<R_{0} \\ =+1, & r>R_{0} .\end{cases}
$$

Thus the divergence at $r=R_{0}$ cancels in (2.44), which implies that the singularity in pressure is integrable. Substituting the interior solution (2.33) and (2.35) into the RHS of (2.43) we have

$$
r^{2} \kappa(r)=\frac{r^{2}}{2} \sqrt{\frac{h}{f}} \frac{d f}{d r}=\frac{4 \pi}{3} \bar{\epsilon} r^{3} \operatorname{sgn}(D)
$$

Taking derivative of (2.46), a straightforward calculation shows that

$$
\frac{d}{d r}\left[r^{2} \kappa(r)\right]=4 \pi \bar{\epsilon} r^{2} \operatorname{sgn}(D)+\frac{8 \pi}{3} \bar{\epsilon} R_{0}^{3} \delta\left(r-R_{0}\right)
$$

which, comparing with the general solution (2.43) and (2.46), reads 


$$
4 \pi \sqrt{\frac{f}{h}} r^{2}\left(\epsilon+p+2 p_{\perp}\right)=4 \pi \sqrt{\frac{f}{h}} r^{2}(\epsilon+3 p)+\frac{8 \pi}{3} \bar{\epsilon} R_{0}^{3} \delta\left(r-R_{0}\right) .
$$

Thus the delta function can be attributed to the difference

$$
8 \pi \sqrt{\frac{f}{h}} r^{2}\left(p_{\perp}-p\right)=\frac{8 \pi \epsilon}{3} R_{0}^{3} \delta\left(r-R_{0}\right)
$$

which indicates an anisotropy in pressure at the singular surface $r=R_{0}$ (see also [16]). It is this $\delta$-function integrable through the Komar formula, together with the relaxation of the isotropic perfect fluid condition at $r=R_{0}$ that provide a physical interpretation of the Schwarzschild star in the regime $R \leq(9 / 8) R_{S}$. From (2.49) the surface energy is found to be

$$
E_{s}=\frac{8 \pi}{3} \epsilon R_{0}^{3}=2 M\left(\frac{R_{0}}{R}\right)^{3},
$$

together with the discontinuity on the surface gravities

$$
\Delta \kappa \equiv \kappa_{+}-\kappa_{-}=\frac{2 M R_{0}}{R^{3}}=\frac{R_{s} R_{0}}{R^{3}}
$$

where

$$
\kappa_{ \pm}= \pm \frac{M R_{0}}{R^{3}}
$$

are equal in magnitude but different in sign. Thus the difference in surface gravities (2.51), between the inner and outer surfaces, provides a surface tension at $r=R_{0}$ which is given by

$$
\tau_{s}=\frac{M R_{0}}{4 \pi R^{3}}=\frac{\Delta \kappa}{8 \pi G} .
$$

In contrast to a black hole, (2.53) corresponds to a physical surface tension (localized in an infinitesimal thin shell at $r=R_{s}$ ) provided by a surface energy and positive transverse pressure as determined by the Komar formula. 


\subsubsection{Discussion}

- The Schwarzschild interior solution, or Schwarzschild star, provides an instructive limiting case of a stellar model in general relativity. Furthermore, in the limit when $R \rightarrow R_{s}^{+}$and $R_{0} \rightarrow R_{s}^{-}$, the Schwarzschild star turns out to be the non-singular gravitational condensate star or gravastar with a negative pressure interior and a surface tension at $R_{s}$, proposed by Mazur and Mottola [60, 61, 62] as an alternative to black holes as the final state of gravitational collapse.

- The surface energy (2.50) is the result of a transverse anisotropic pressure $T_{\theta}^{\theta}=$ $T_{\phi}^{\phi}=p_{\perp}$. There is no energy-momentum tensor $T_{t t}$ at the surface.

- The gravastar solution, or Schwarzschild-de Sitter interior solution, with $p=-\epsilon$ has zero entropy density $s=0$. Thus a gravastar is a zero temperature system, corresponding to a condensate state.

- The non-analytic cusp behavior of the metric functions $f(r)$ and $h(r)$ (see figures 2.5, 2.6 and 2.8), invalidates the analytic continuation in the Schwarzschild black hole vacuum; therefore it does not require any periodicity in imaginary time.

- The Killing vector $K_{\mu}$ remains timelike for a gravastar, therefore $t$ is a global time. This property is essential to develop the correct time unitary evolution in quantum mechanics. 


\title{
Chapter 3
}

\section{SLOWLY ROTATING RELATIVISTIC COMPACT OBJECTS}

\author{
The general theory of relativity is a theory of gravitation; \\ and like the Newtonian theory of gravitation, \\ which it refines and broadens, \\ its natural home is astronomy
}

S. Chandrasekhar

\section{INTRODUCTION}

In this chapter, we review the equations of structure, first derived by Hartle [33], which govern the properties of equilibrium configurations of slowly rotating compact objects $^{1}$ in general relativity. Hartle \& Thorne [36] integrated Hartle's equations numerically for the case of particular equations of state of white dwarfs and neutron stars. Chandrasekhar \& Miller [21] investigated, numerically, surface and integral properties for a slowly rotating homogeneous star of constant energy density. The formalism introduced in this chapter is essential to understand the results which will be discussed in the last chapter.

The Hartle perturbative model is constructed under the following assumptions:

- The matter of the configuration satisfies a one-parameter equation of state (EOS) $p=p(\epsilon)$, where $p$ is the pressure and $\epsilon$ is the mass-energy density.

\footnotetext{
${ }^{1}$ For compact object we follow the definition given in [93]; white dwarfs, neutron stars, gravastars, which are the remaining of a star when it consumes its nuclear fuel (see also section 1.3).
} 
- Given an EOS the relativistic hydrostatic equilibrium equations are solved for a non-rotating spherically symmetric configuration.

- The configuration is set in slow and uniform rotation. Hartle \& Sharp [35] showed that configurations rotating uniformly minimize the total mass-energy. On the other hand, in slow rotation, fractional changes in energy density and pressure are less than unity. This condition implies

$$
R \Omega<<1
$$

where $R$ is the radius of the configuration and $\Omega$ is its angular velocity.

- We restrict to axially symmetric objects, with mass quadrupole moments independent of time. Deviation from this condition would imply emission of gravitational waves and a non-equilibrium situation.

- The small fractional changes are considered as perturbations on the non-rotating configuration. The Einstein equations are expanded to second order in the angular velocity $\Omega$.

The chapter is organized as follows. In the next section, we discuss the dragging of inertial frames manifested in axisymmetric space-times. In sections 3.2, 3.3 and 3.4 we assemble the general-relativistic equations of structure for slowly rotating masses. The application of the Hartle structure equations for a slowly rotating Schwarzschild star will be discussed in section 3.5.

\subsection{Rotational 'DRAGGING' OF INERTIAL FRAMES}

The appropriate line element, and the one used in $[33,36]$, for this situation is ${ }^{2}$

\footnotetext{
${ }^{2}$ The subscript $(0)$ in the metric functions denotes quantities in the static configuration, except for the functions $h_{0}$ and $m_{0}$ which correspond to the $l=0$ term in the harmonic expansion.
} 


$$
\begin{aligned}
d s^{2}=-\mathrm{e}^{2 \nu_{0}}[1+ & \left.2 h_{0}(r)+2 h_{2}(r) P_{2}(\cos \theta)\right] d t^{2} \\
+ & \mathrm{e}^{2 \lambda_{0}}\left\{1+\frac{\mathrm{e}^{2 \lambda_{0}}}{r}\left[2 m_{0}(r)+2 m_{2}(r) P_{2}(\cos \theta)\right]\right\} d r^{2} \\
+ & r^{2}\left[1+2 k_{2}(r) P_{2}(\cos \theta)\right]\left\{d \theta^{2}+[d \phi-\omega(r) d t]^{2} \sin ^{2} \theta\right\},
\end{aligned}
$$

where $h_{0}, h_{2}, m_{0}, m_{2}, k_{2}$ are quantities of order $\Omega^{2}$, and $P_{2}(\cos \theta)=\left(3 \cos ^{2} \theta-1\right) / 2$ is the Legendre polynomial of order 2. The quantity $\omega$, which is of the order of the angular velocity of the star $\Omega$, is a function of $r$ that describes the dragging of the inertial frames. In the non-rotating case the metric (3.2) reduces to the static form

$$
d s^{2}=-e^{2 \nu_{0}} d t^{2}+e^{2 \lambda_{0}} d r^{2}+r^{2}\left(d \theta^{2}+\sin ^{2} \theta d \phi^{2}\right)
$$

The 'dragging' effect is a purely relativistic effect, which in general can be described by two ways_ effects on gyroscopes and the cumulative effect on the motion of particles. We concentrate on the cumulative dragging which it is worthwhile to discuss. We follow the discussion given by Thorne [100].

Let us imagine a distant observer, far from a rotating star. Let the observer throw a test particle with zero angular momentum, or Zero Angular Momentum Observer (ZAMO), relative to the axis of rotation of the star. When the test particle approaches the star, its angular momentum is still zero, but due to the dragging of the inertial frames, it will have a non-zero angular velocity. The magnitude of the dragging is found to be

$$
\omega=\frac{d \phi}{d t}=\frac{p^{\phi}}{p^{t}}=\frac{g^{\phi t} p_{t}}{g^{t t} p_{t}}=\frac{g^{\phi t}}{g^{t t}}=-\frac{g_{\phi t}}{g_{\phi \phi}} .
$$

Thus the function $\omega(r)$ depends only on the local geometry. Some authors $[33,36]$ call this $\omega(r)$, the angular velocity of the local ZAMO relative to a distant observer. 
Let the star rotate uniformly with angular velocity $\Omega$. Moreover, we assume that there are no convective motions such that $u^{\theta}=u^{r}=0$. Let us find the four-velocity components $u^{\mu}$ which satisfy the normalization condition

$$
u^{\mu} u_{\mu}=-1
$$

Equation (3.5) reduces to

$$
g_{t t}\left(u^{t}\right)^{2}+2 g_{t \phi} u^{t} u^{\phi}+\Omega^{2} g_{\phi \phi}\left(u^{t}\right)^{2}=-1
$$

where we used $u^{\phi}=\Omega u^{t}$. Simplifying (3.6) we obtain

$$
\begin{aligned}
u^{t} & =\left(-g_{t t}-2 \Omega g_{t \phi}-g_{\phi \phi} \Omega^{2}\right)^{-1 / 2} \\
& =\mathrm{e}^{-\nu_{0}}\left[1+\frac{1}{2} r^{2} \sin ^{2} \theta(\Omega-\omega)^{2} \mathrm{e}^{-\nu_{0} / 2}-h_{0}-h_{2} P_{2}(\cos \theta)\right] .
\end{aligned}
$$

It is customary to define the quantity

$$
\varpi \equiv \Omega-\omega
$$

to be the angular velocity of the fluid as measured by the local ZAMO. In general, centrifugal effects will be determined by this quantity. The equation which governs $\varpi$ can be obtained from the $(t, \phi)$ component of the Einstein equations

$$
R_{\phi}^{t}=8 \pi T_{\phi}^{t}
$$

Considering the 4-velocity components (3.7) the energy-momentum tensor reads

$$
T_{\phi}^{t}=(\epsilon+p)\left(u^{t}\right)^{2}\left(g_{t \phi}+\Omega g_{\phi \phi}\right) \text {. }
$$


Due to the axial symmetry of the problem, quantities like the mass-energy density and the metric coefficients are preserved under a reversal in the direction of rotation $\phi \rightarrow-\phi$ as a reversal in time $t \rightarrow-t$ (see section 1.4). Thus, only even powers will appear in an expansion in terms of $\Omega$. On the other hand, the dragging function $\omega(r)$ will have only odd powers in the same expansion. Therefore, to study effects at order $\Omega^{2}$, for the function $\omega$ it is sufficient to consider only terms at order $\Omega$.

Using the relevant components of the Einstein tensor (see Appendix B), we calculate at first order in $\Omega$ the Einstein equation components (3.9) and (3.10) to obtain

$$
\frac{d}{d r}\left(r^{4} j \frac{d \varpi}{d r}\right)+4 r^{3} \frac{d j}{d r} \varpi=0,
$$

where

$$
j(r) \equiv \mathrm{e}^{-\left(\lambda_{0}+\nu_{0}\right)} .
$$

Thus $\varpi$ can be found by integrating (3.11) from the origin, where the solution is regular, up to the boundary of the configuration. In the exterior empty region $r>R$, $j(r)=1$ and (3.11) can be easily integrated to give

$$
\varpi(r)=\Omega-\frac{2 J}{r^{3}},
$$

where the constant $J$ corresponds to the angular momentum of the star [33, 100]. Equation (3.11) will be integrated outward from the origin with the boundary conditions $\varpi(0)=\varpi_{c}=$ const., and $d \varpi / d r=0$. The value of $\varpi_{c}$ is chosen arbitrarily. Once the solution on the surface is found, one can determine the angular momentum $J$ and the angular velocity $\Omega$ using the equations

$$
J=\frac{1}{6} R^{4}\left(\frac{d \varpi}{d r}\right)_{r=R}, \quad \Omega=\varpi(R)+\frac{2 J}{R^{3}} .
$$

The angular momentum is related linearly to $\Omega$ through the relation 


$$
J=I \Omega
$$

where $I$ is the relativistic moment of inertia for slowly rotating objects. In contrast to the Newtonian case, the relativistic moment of inertia depends not only on the rest mass of the star but also of the mass-energy associated to the compression of the matter to certain density and the effective energy of the gravitational interaction of different parts of the star.

Hartle [33] showed that the rate of change of $\varpi(r)$ always has the same sign of $\Omega$. Moreover, $|\varpi(r)|$ is a decreasing function of $r$; therefore the dragging effects are the largest near to the center of the star.

\subsection{Rotational PERTURBATIONS IN MASS-ENERGY AND PRESSURE}

Due to the rotation, the star will deform carrying with it changes in pressure and energy density. For a given $\operatorname{EOS} \epsilon=\epsilon(p)$ the hydrodynamic equilibrium equation (2.5) can be recast in the form

$$
p_{, j}=(\epsilon+p)\left[\log \frac{e^{-\nu}}{\left(1-V^{2}\right)^{1 / 2}}\right]_{, j} \quad(j=r, \theta),
$$

here the comma indicates partial derivative $p_{, \nu}=\partial p / \partial \nu$ and

$$
V=e^{\psi-\nu} \varpi
$$

where we have introduced the identifications

$$
\begin{aligned}
& e^{\psi}=r \sin \theta\left[1+k_{2}(r) P_{2}(\cos \theta)\right] \\
& e^{\nu}=e^{\nu_{0}}\left[1+h_{0}(r)+h_{2}(r) P_{2}(\cos \theta)\right] .
\end{aligned}
$$

Integrating equation (3.17) we obtain 


$$
\nu+\frac{1}{2} \log \left(1-V^{2}\right)+P=\text { const },
$$

where

$$
P=\log (\epsilon+p)-\int_{\epsilon_{c}}^{\epsilon} \frac{d \epsilon}{\epsilon+p}
$$

which satisfies the expansion ${ }^{3}$

$$
P=P_{0}(r)+\delta P_{0}(r)+\delta P_{2}(r) P_{2}(\cos \theta)
$$

Expanding (3.17) at first order in $\Omega$ we have

$$
V(r, \theta)=e^{-\nu_{0}} \varpi r \sin \theta+\mathcal{O}\left(\Omega^{2}\right)
$$

Thus, using expansion (3.22) into (3.19) we have the set of equations

$$
\begin{gathered}
\delta P_{0}=-h_{0}+\frac{1}{3} r^{2} e^{-2 \nu_{0}} \varpi^{2}+C\left(\Omega^{2}\right), \quad(l=0) \\
\delta P_{2}=-h_{2}(r)-\frac{1}{3} r^{2} e^{-2 \nu_{0}} \varpi^{2}, \quad(l=2)
\end{gathered}
$$

where $C$ is a constant of order $\Omega^{2}$. Thus equations (3.23) and (3.24) determine the perturbations in pressure and mass-energy density. We will return to these equations when we perform the numerical integrations.

\section{$3.3 l=0$ EQUATIONS - SPHERICAL DEFORMATIONS OF THE STAR}

In general relativity it is expected that, due to the rotation, the mass of a spinning star will be different from its corresponding value in the static case. To determine such change, the $l=0$ equations in the harmonic expansion are used. Let us recall

${ }^{3}$ I hope not to confuse the reader with the notation used for $P_{2}$. Here $\delta P_{2}$ indicates the second order in the harmonic expansion, and $P_{2}(\cos \theta)$ corresponds to the Legendre polynomial. 
that for a spherically symmetric configuration, the mass $M$, as measured by a distant observer, is given by the $g_{t t}$ component of the metric

$$
g_{t t} \rightarrow-\left(1-\frac{2 M}{r}\right), \quad r \rightarrow \infty
$$

For this specific problem, it is sufficient to calculate the rotational perturbations $m_{0}$ and $\delta P_{0}$. Note that equation (3.23) already provides a first integral for the $l=0$ field equations. Additionally the field equations components $G_{\mu}{ }^{\nu}=8 \pi T_{\mu}{ }^{\nu}$ to be calculated, and chosen in [33], are (see Appendix B)

$$
\begin{aligned}
& R_{t}^{t}-\frac{1}{2} R \delta_{t}^{t}=8 \pi T_{t}^{t} \\
& R_{r}^{r}-\frac{1}{2} R \delta_{r}^{r}=8 \pi T_{r}^{r} .
\end{aligned}
$$

Using the second order contributions to $G_{\mu}{ }^{\nu}$ found in [33], the Einstein equations reduces to

$$
\begin{gathered}
\frac{d m_{0}}{d r}=4 \pi r^{2}(\epsilon+p) \frac{d \epsilon}{d p} \delta P_{0}+\frac{1}{12} r^{4} j^{2}\left(\frac{d \varpi}{d r}\right)^{2}-\frac{1}{3} r^{3} \varpi^{2} \frac{d j^{2}}{d r} \\
\frac{d h_{0}}{d r}=-\frac{d}{d r} \delta P_{0}+\frac{1}{3} \frac{d}{d r}\left(r^{2} \mathrm{e}^{-2 \nu_{0}} \varpi^{2}\right) \\
=m_{0} e^{4 \lambda_{0}}\left(\frac{1}{r^{2}}+8 \pi p\right)-\frac{1}{12} e^{2 \lambda_{0}} r^{3} j^{2}\left(\frac{d \varpi}{d r}\right)^{2}+4 \pi r e^{2 \lambda_{0}}(\epsilon+p) \delta P_{0} .
\end{gathered}
$$

These equations will be integrated outward from the origin, where the boundary conditions $h_{0}(0)=m_{0}(0)=0$ must be satisfied. In this approximation, the slowly rotating configuration will have the same central pressure as in the static case. In the exterior region

$$
\epsilon=p=0, \quad r>R
$$


thus (3.28) and (3.29) can be integrated explicitly to give

$$
\begin{gathered}
m_{0}=\delta M-\frac{J^{2}}{r^{3}}, \\
h_{0}=-\frac{\delta M}{r-2 M_{0}}+\frac{J^{2}}{r^{3}\left(r-2 M_{0}\right)},
\end{gathered}
$$

where $M_{0}$ corresponds to the total mass of the star and $\delta M$ is an integration constant which is associated to the change in mass due to the rotation. The constant $\delta M$ will be found by matching the interior and exterior solutions for $h_{0}$ at the boundary $r=R$

$$
\delta M=m_{0}(R)+\frac{J^{2}}{R^{3}}
$$

\subsubsection{AMENDED CHANGE OF MASS}

Recently Reina \& Vera $[84,85]$ revisited Hartle's framework within the context of the modern theory of perturbed matchings [56]. They found that the perturbative functions at first and second order are continuous across the boundary of the configuration except when the energy density is discontinuous there. In this particular case, the discontinuity in the radial function $m_{0}$ at the boundary is proportional to the energy density there. Furthermore, Reina and Vera showed that the manifestation of this jump in the perturbative function $m_{0}$ induces a modification to the original change of mass (3.33), which is given by [84]

$$
\begin{aligned}
\delta M & =\delta M^{H}+\delta M^{C} \\
& =\left[m_{0}(R)+\frac{J^{2}}{R^{3}}\right]+4 \pi \frac{R^{3}}{M_{0}}\left(R-2 M_{0}\right) \epsilon(R) \delta p_{0}(R) .
\end{aligned}
$$

where $\delta M^{H}$ corresponds to the original change of mass (3.33) and $\delta M^{C}$ is the correction term. Reina and Vera point out that this correction is relevant in configurations where the energy density does not vanish at the boundary, for instance, strange 
quark stars [64] and constant density (homogeneous) masses. We shall consider the corrected expression (3.34) in our computations.

\section{$3.4 l=2$ EQUATIONS - QUADRUPOLE DEFORMATIONS OF THE STAR}

Due to the rotation, the surface of the Schwarzschild star will be deformed from the spherical shape it has in the static case, preserving the same central density. The modified radius of the slowly rotating isobaric surface is given by

$$
r(\theta)=r_{0}+\xi_{0}\left(r_{0}\right)+\xi_{2}\left(r_{0}\right) P_{2}(\cos \theta),
$$

where $r_{0}$ corresponds to the radius of the spherical surface in the non-rotating case, and the deformations $\xi_{0}$ and $\xi_{2}$ satisfy

$$
\delta p_{0}=-\left(\frac{1}{\epsilon+p} \frac{d p}{d r}\right)_{0} \xi_{0}\left(r_{0}\right), \quad \delta p_{2}=-\left(\frac{1}{\epsilon+p} \frac{d p}{d r}\right)_{0} \xi_{2}\left(r_{0}\right) .
$$

To determine completely the $l=2$ solution, we must find the solutions for the functions $\xi_{2}(r), h_{2}(r), k_{2}(r)$ and $m_{2}(r)$. The quadrupole deformations of the star, as given by the $l=2$ field equations, were calculated in $[21,33]$. Here we just summarize the main results

$$
\begin{gathered}
\frac{d v_{2}}{d r}=-2 \frac{d \nu_{0}}{d r} h_{2}+\left(\frac{1}{r}+\frac{d \nu_{0}}{d r}\right)\left[\frac{1}{6} r^{4} j^{2}\left(\frac{d \varpi}{d r}\right)^{2}-\frac{1}{3} r^{3} \varpi^{2} \frac{d j^{2}}{d r}\right] . \\
\frac{d h_{2}}{d r}=-\frac{2 v_{2}}{r[r-2 m(r)]\left(d \nu_{0} / d r\right)} \\
+\left\{-2 \frac{d \nu_{0}}{d r}+\frac{r}{2[r-2 m(r)]\left(d \nu_{0} / d r\right)}\left[8 \pi(\epsilon+p)-\frac{4 m(r)}{r^{3}}\right]\right\} h_{2} \\
+\frac{1}{6}\left[r \frac{d \nu_{0}}{d r}-\frac{1}{2[r-2 m(r)]\left(d \nu_{0} / d r\right)}\right] r^{3} j^{2}\left(\frac{d \varpi}{d r}\right)^{2} \\
-\frac{1}{3}\left[r \frac{d \nu_{0}}{d r}+\frac{1}{2[r-2 m(r)]\left(d \nu_{0} / d r\right)}\right] r^{2} \varpi^{2} \frac{d j^{2}}{d r},
\end{gathered}
$$


where $v_{2}=h_{2}+k_{2}$. These equations will be integrated outward from the center, where $h_{2}=v_{2}=0$. Outside the star, (3.37) and (3.38) are integrated analytically

$$
\begin{gathered}
h_{2}(r)=J^{2}\left(\frac{1}{M_{0} r^{3}}+\frac{1}{r^{4}}\right)+K Q_{2}^{2}\left(\frac{r}{M_{0}}-1\right), \\
v_{2}(r)=-\frac{J^{2}}{r^{4}}+K \frac{2 M_{0}}{\left[r\left(r-2 M_{0}\right)\right]^{1 / 2}} Q_{2}^{1}\left(\frac{r}{M_{0}}-1\right),
\end{gathered}
$$

where $K$ is an integration constant and $Q_{n}^{m}$ are the associated Legendre functions of the second kind with argument $\xi=\left(r / M_{0}\right)-1$, which are given by

$$
\begin{gathered}
Q_{2}^{1}(\xi)=\left(\xi^{2}-1\right)^{1 / 2}\left[\frac{3 \xi^{2}-2}{\xi^{2}-1}-\frac{3}{2} \xi \log \left(\frac{\xi+1}{\xi-1}\right)\right], \\
Q_{2}^{2}(\xi)=\frac{3}{2}\left(\xi^{2}-1\right) \log \left(\frac{\xi+1}{\xi-1}\right)-\frac{3 \xi^{3}-5 \xi}{\xi^{2}-1} .
\end{gathered}
$$

The integration constant $K$ in (3.39) and (3.40) will be found by the matching, at the boundary of the star $r=R$, with the numerical solutions of (3.37) and (3.38). Once the functions $h_{2}$ and $v_{2}$ have been found from (3.37) and (3.38), the perturbations factors $m_{2}$ and $\delta p_{2}$ are determined from the relations

$$
\begin{gathered}
m_{2}=[r-2 m(r)]\left[-h_{2}(r)-\frac{1}{3} r^{3}\left(\frac{d j^{2}}{d r}\right) \varpi^{2}+\frac{1}{6} r^{4} j^{2}\left(\frac{d \varpi}{d r}\right)^{2}\right], \\
\delta p_{2}=-h_{2}(r)-\frac{1}{3} r^{2} \varpi^{2} e^{-2 \nu_{0}} .
\end{gathered}
$$

Thus the rotational deformation of the star (3.35) is completely determined. Note that equation (3.35) determines the level surfaces in some particular coordinate system. However, an invariant definition of the isobaric surfaces can be given by doing an embedding (see e.g. [14]) in a flat three-dimensional flat space. We look for the three dimensions surface in flat space, with spherical coordinates $\left(r^{*}, \theta^{*}, \phi^{*}\right)$, which has the same intrinsic geometry as the level surface we are considering. The 3-surface in flat 
space, at order $\Omega^{2}$, which satisfy the conditions above corresponds to the Mclaurin spheroid $[6,33,100]$

$$
r^{*}\left(\theta^{*}\right)=r+\xi_{0}(r)+\left[\xi_{2}(r)+r\left(v_{2}-h_{2}\right)\right] P_{2}\left(\cos \theta^{*}\right) .
$$

We introduce the ellipticity of the spheroid [21, 65]

$$
\varepsilon=\frac{\text { equatorial radius }- \text { polar radius }}{\text { mean radius }}=-\frac{3}{2 r}\left[\xi_{2}+r\left(v_{2}-h_{2}\right)\right]
$$

which is valid to order $\Omega^{2}$.

\subsubsection{EXTERNAL GRAVITATIONAL FIELD}

The metric perturbations $\left(h_{2}, v_{2}, m_{2}\right)$ determine the deformations of the external gravitational field of a slowly rotating star. In the asymptotically flat region, the star's mass quadrupole moment is given by [36]

$$
Q=\frac{J^{2}}{M_{0}}+\frac{8}{5} K M_{0}^{3}
$$

Here the constant $K$ is the same constant that appears in equations (3.39) and (3.40). Once the numerical solutions for (3.38) and (3.37) have been matched to the exterior solutions at the boundary $r=R$, the numerical value of the constant $K$ can be found and the quadrupole moment can be completely determined.

With the metric perturbations determined we can write the exterior metric of a slowly rotating star (3.2), to second order in $\Omega$. Using equations (3.31), (3.32), (3.39), (3.40) and (3.47) we find the following 


$$
\begin{aligned}
d s^{2}=-\left(1-\frac{2 \mu}{r}+\right. & \left.\frac{2 J^{2}}{r^{4}}\right)\left\{1+2\left[\frac{J^{2}}{\mu r^{3}}\left(1+\frac{\mu}{r}\right)\right.\right. \\
& \left.\left.+\frac{5}{8} \frac{Q-J^{2} / \mu}{\mu^{3}} Q_{2}{ }^{2}\left(\frac{r}{\mu}-1\right)\right] P_{2}(\cos \theta)\right\} d t^{2} \\
& +\left(1-\frac{2 \mu}{r}+\frac{2 J^{2}}{r^{4}}\right)^{-1}\left\{1-2\left[\frac{J^{2}}{\mu r^{3}}\left(1-\frac{5 \mu}{r}\right)\right.\right. \\
+r^{2}\{1+2[- & \left.\left.\frac{J^{2}}{\mu r^{3}}\left(1+\frac{2 \mu}{r}\right)+\frac{5}{8} \frac{Q-J^{2} / \mu}{\mu^{3}} Q_{2}{ }^{2}\left(\frac{r}{\mu}-1\right)\right] P_{2}(\cos \theta)\right\} d r^{2} \\
- & \left.\left.\left.Q_{2}{ }^{2}\left(\frac{r}{\mu}-1\right)\right\}\right] P_{2}(\cos \theta)\right\}\left[d \theta^{2}+\sin ^{2} \theta\left(d \phi-\frac{2 J}{r^{3}} d t\right)^{2}\right],
\end{aligned}
$$

where $\mu \equiv M_{0}+\delta M$. It is instructive to compare this metric with the solution for a stationary and axisymmetric space-time, as given by the Kerr metric (1.40). However, we must express it in the coordinates of (3.48) through the transformation [36]

$$
\begin{gathered}
r \rightarrow r\left\{1-\frac{a^{2}}{2 r^{2}}\left[\left(1+\frac{2 \mu}{r}\right)\left(1-\frac{\mu}{r}\right)+\cos ^{2} \theta\left(1-\frac{2 \mu}{r}\right)\left(1+\frac{3 \mu}{r}\right)\right]\right\} \\
\theta \rightarrow \theta-\frac{a^{2}}{2 r^{2}}\left(1+\frac{2 \mu}{r}\right) \sin \theta \cos \theta
\end{gathered}
$$

Thus it can be seen that the Kerr metric satisfies the condition

$$
J=\mu a, \quad Q=\frac{J^{2}}{\mu}, \quad \text { where } \quad K=0
$$

These parameters are essential to identify the Kerr space-time, at order $\Omega^{2}$. Moreover, we will use the result (3.51) to compare with the exterior space-time of a slowly rotating Schwarzschild star. This relation for the quadrupole moment can also be observed from the following behaviors of (3.41) and (3.42)

$$
Q_{2}^{1}\left(\frac{r}{\mu}-1\right) \approx\left[2\left(\frac{r}{\mu}-2\right)\right]^{-1 / 2}, \quad Q_{2}^{2}\left(\frac{r}{\mu}-1\right) \approx\left(\frac{r}{\mu}-2\right)^{-1 / 2}
$$


which are divergent when $r$ approaches the Schwarzschild radius $r_{S}=2 \mu$. Therefore at this limit, the rotational and quadrupole deformations are infinite. Thus in order to have regular solutions at this limit the Kerr condition $K=0$, which leads to (3.51), must be satisfied.

\subsection{Structure equations for the Schwarzschild star}

In a seminal paper, Chandrasekhar \& Miller [21] applied the Hartle's structure equations to study slowly and uniformly rotating masses characterized by a constant energy density $\epsilon$. In that article, surface and integral properties were calculated numerically for several values of the compactness parameter $R / R_{s}$, where $R$ is the radius of the star and $R_{s}$ is the Schwarzschild radius. This procedure might be interpreted as a quasi-stationary contraction of the star [65]. In a quasi-stationary collapse, some of the gravitational potential energy is converted into internal energy, with the rest being radiated away. Thus the total gravitational mass $M$ decreases, but the rest mass $M_{0}$ is kept constant.

The geometry of the Schwarzschild interior solution, or Schwarzschild star, was discussed in section 2.3. To facilitate the numerical integrations, it is useful to introduce the variables [21]

$$
r=\left(1-y^{2}\right)^{1 / 2}, \quad y_{1}^{2}=1-\frac{R^{2}}{\alpha^{2}}=1-H^{2} R^{2}
$$

Here $r$ is being measured in the unit $\alpha=1 / H$, where $H^{2} \equiv \frac{8 \pi \epsilon}{3}$ was defined in (2.8). In terms of (3.53) the Schwarzschild star solution (2.27), (2.33), (2.34) and (2.35) takes the form

$$
\mathrm{e}^{\lambda_{0}}=\frac{1}{y}, \quad \mathrm{e}^{\nu_{0}}=\frac{1}{2}\left(3 y_{1}-y\right)
$$




$$
\begin{gathered}
\frac{p}{\epsilon}=\frac{y-y_{1}}{3 y_{1}-y}, \\
j=\frac{2 y}{3 y_{1}-y}, \quad y^{2}=1-\frac{2 m(r)}{r} .
\end{gathered}
$$

For future purposes we introduce the parameter

$$
k \equiv 3 y_{1}-1
$$

As we discussed in section 2.3, the Schwarzschild interior solution shows a divergence in pressure at the radius $R_{B}=(9 / 8) R_{S}$ or Schwarzschild-Buchdahl bound. In terms of the parameter $k$, defined above, this limit is equivalent to the condition

$$
y_{1}>\frac{1}{3}
$$

for the solution to be regular. We will go back to this point in the next chapter. Following [21], it is convenient to introduce the coordinate

$$
x \equiv 1-y=1-\left[1-\left(\frac{r}{\alpha}\right)^{2}\right]^{1 / 2},
$$

where $x$ covers the range $(0,2 / 3]$. From $(3.59)$ we obtain the transformation

$$
\frac{d}{d r}=\frac{1}{\alpha} \frac{\sqrt{x(2-x)}}{1-x} \frac{d}{d x} .
$$

In terms of (3.59), and using (3.60), the equation for $\varpi$ (3.11) takes the form

$$
x\left[2 k+(2-k) x-x^{2}\right] \frac{d^{2} \varpi}{d x^{2}}+\left[5 k+(3-5 k) x-4 x^{2}\right] \frac{d \varpi}{d x}-4(k+1) \varpi=0 .
$$

The behavior near the origin can be seen as follows: where $x \approx 0$, from (3.61) we have 


$$
5 k \frac{d \varpi}{d x}-4(k+1) \varpi=0
$$

which gives the integral

$$
\int_{\varpi_{c}}^{\varpi} \frac{d \varpi}{\varpi}=\int_{0}^{x} \frac{4(k+1)}{5 k} d x
$$

Evaluating the integral, keeping lower order terms in $x$, we have finally

$$
\varpi=\left[1+\frac{4(k+1)}{5 k} x\right] \varpi_{c},
$$

where $\varpi$ is measured in the unit $\varpi_{c}$, its value at the center, which is arbitrary. Similarly the field equation for the function $m_{0}$ (3.28) take the form

$$
\frac{d m_{0}}{d x}=\alpha^{3} \frac{(1-x)[x(2-x)]^{3 / 2}}{(k+x)^{2}}\left[\frac{1}{3} x(2-x)\left(\frac{d \varpi}{d x}\right)^{2}+\frac{8(k+1)}{3(k+x)} \varpi^{2}\right] .
$$

To analyze the near origin behavior $(x \approx 0)$ for $(3.65)$, from $(3.64)$ we use the following results

$$
\varpi^{2} \approx\left[1+\frac{8(k+1)}{5 k} x\right] \varpi_{c}^{2}+\mathcal{O}\left(x^{2}\right), \quad \frac{d \varpi}{d x}=\frac{4(k+1)}{5 k} \varpi_{c} .
$$

Substituting (3.66) into (3.65) and simplifying we have

$$
\frac{1}{\alpha^{3}} \frac{d m_{0}}{d x}=\frac{2 \sqrt{2} x^{3 / 2}}{(k+x)^{2}}\left\{\frac{8(k+1)}{3(k+x)}\left[1+\frac{8(k+1)}{5 k} x\right]\right\} \varpi_{c}^{2},
$$

where we have used the expansion

$$
x^{3 / 2}(1-x)(2-x)^{3 / 2} \approx 2 \sqrt{2} x^{3 / 2}+\mathcal{O}\left(x^{5 / 2}\right) .
$$

After some algebra (3.67) is reduced to

$$
\frac{1}{\alpha^{3}} \frac{d m_{0}}{d x}=\left[\frac{16 \sqrt{2}}{3 k^{3}}(k+1) x^{3 / 2}\right] \varpi_{c}^{2}
$$


which can be easily integrated to obtain

$$
m_{0}=\left[\frac{32 \sqrt{2}(k+1)}{15 k^{3}} x^{5 / 2}\right] \alpha^{3} \varpi_{c}^{2}+\mathcal{O}\left(x^{7 / 2}\right) .
$$

The equation for the perturbation pressure factor $\delta P_{0}$, as a function of $x$, can be obtained from (3.29)

$$
\begin{aligned}
& \frac{d}{d x} \delta P_{0}=-\frac{(k+1)}{(1-x)(k+x)} \delta P_{0}-\left[\frac{2+(k+1)(1-x)-3(1-x)^{2}}{(k+x)(1-x)^{2}[x(2-x)]^{3 / 2}}\right] \alpha^{-1} m_{0} \\
& \quad+\frac{8 x(2-x)}{3(k+x)^{2}} \varpi\left(\frac{d \varpi}{d x}\right)+\frac{[x(2-x)]^{2}}{3(1-x)(k+x)^{2}}\left(\frac{d \varpi}{d x}\right)^{2}-\frac{8}{3}\left[\frac{1-(k+1)(1-x)}{(k+x)^{3}}\right] \varpi^{2} .
\end{aligned}
$$

Near the origin $(x \approx 0),(3.71)$ reduces to

$$
\frac{1}{\alpha^{2}} \frac{d}{d x} \delta P_{0}=-\frac{8}{3}\left[\frac{1-(k+1)}{k^{3}}\right] \varpi^{2},
$$

which, after using (3.66), can be integrated to give

$$
\delta P_{0}=\left(\frac{8 x}{3 k^{2}}\right) \alpha^{2} \varpi_{c}^{2} .
$$

The equations (3.38) and (3.37) for $h_{2}$ and $v_{2}$, as function of $x$, take the form

$$
\begin{gathered}
\frac{d v_{2}}{d x}=-\frac{2 h_{2}}{k+x}+\frac{2[x(2-x)]^{2}}{3(k+x)^{3}}[k(1-x)+x(3-2 x)] \times \\
{\left[\left(\frac{d \varpi}{d x}\right)^{2}+\frac{4(k+1)}{x(2-x)(k+x)} \varpi^{2}\right] \alpha^{2},} \\
\begin{aligned}
\frac{d h_{2}}{d x}=\frac{(1-x)^{2}+(k+1)(1-x)-2}{x(2-x)(k+x)} h_{2}-\frac{2(k+x)}{[x(2-x)]^{2}} v_{2} \\
+\frac{\alpha^{2}}{3}\left\{2[x(2-x)]^{2}-(k+x)^{2}\right\} \frac{x(2-x)}{(k+x)^{3}}\left(\frac{d \varpi}{d x}\right)^{2} \\
+\frac{4 \alpha^{2}}{3}(k+1)\left[2 x^{2}(2-x)^{2}+(k+x)^{2}\right] \frac{\varpi^{2}}{(k+x)^{4}} .
\end{aligned}
\end{gathered}
$$


The behavior near the origin for (3.74) gives

$$
\frac{d v_{2}}{d x}=-\frac{2 h_{2}}{k}\left(1-\frac{x}{k}\right)+\mathcal{O}\left(x^{2}\right)
$$

Similarly for (3.75), after some algebra, we obtain

$$
h_{2}=\frac{(k+x)(x+1)}{x} v_{2}
$$

Substituting (3.77) into (3.76) and integrating we have

$$
h_{2}=\frac{4(k+1)}{3 k^{2}} x=a x .
$$

Now that we have obtained (3.78), we can substitute back into (3.76) so after integration we obtain

$$
v_{2}=-\frac{4(k+1)}{3 k^{3}} x^{2}=b x^{2}
$$

Thus, the constants $a$ and $b$ are related through the relation

$$
k^{2} a-k^{3} b=\frac{8}{3}(k+1) .
$$

Additionally we have the equations (3.23) and (3.24) for the pressure perturbation factors $\delta P_{0}$ and $\delta P_{2}$, which in this context take the forms

$$
\begin{gathered}
h_{0}-\frac{4 x(2-x)}{3(k+x)^{2}} \varpi^{2}+\delta P_{0}=\text { const. } \\
h_{2}+\frac{4 x(2-x)}{3(k+x)^{2}} \varpi^{2}+\delta P_{2}=0
\end{gathered}
$$

The functions $\delta P_{0}, \delta P_{2}, h_{2}, k_{2}$ and $v_{2}$ are measured in the unit $\alpha^{2} \omega_{c}^{2}$ and $m_{0}$ is measured in the unit $\alpha^{3} \omega_{c}^{2}$. Solutions to (3.74) and (3.75) can be expressed as the superposition of a particular and a complementary solution 


$$
h_{2}=h_{2}^{(p)}+A h_{2}^{(c)}, \quad v_{2}=v_{2}^{(p)}+A v_{2}^{(c)}
$$

with $\beta$ being an integration constant. The complementary functions here satisfy the homogeneous forms of equations (3.74) and (3.75)

$$
\begin{gathered}
\frac{d v_{2}^{(c)}}{d x}=-\frac{2 h_{2}^{(c)}}{k+x}, \\
\frac{d h_{2}^{(c)}}{d x}=\frac{(1-x)^{2}+(k+1)(1-x)-2}{x(2-x)(k+x)} h_{2}^{(c)}-\frac{2(k+x)}{[x(2-x)]^{2}} v_{2}^{(c)},
\end{gathered}
$$

which have the following behaviors near the origin

$$
h_{2}^{(c)}=-k B x, \quad v_{2}^{(c)}=B x^{2}
$$

where $B$ is an arbitrary constant. Finally the exterior solutions (3.13), (3.31), (3.32), (3.39) and (3.40), as a function of the variable $y_{1}$, take the following forms ${ }^{4}$

$$
\begin{gathered}
\varpi=\Omega-\frac{2 J}{r^{3}}, \\
m_{0}(R)=\delta M-\frac{J^{2}}{r^{3}}, \\
h_{0}=-\frac{m_{0}}{r-\left(1-y_{1}^{2}\right)^{3 / 2}}, \\
h_{2}=\left[\frac{2}{\left(1-y_{1}^{2}\right)^{3 / 2}}+\frac{1}{r}\right] \frac{J^{2}}{r^{3}}+K Q_{2}^{2}, \\
v_{2}=-\frac{J^{2}}{r^{4}}+K \frac{\left(1-y_{1}^{2}\right)^{3 / 2}}{\left\{r\left[r-\left(1-y_{1}^{2}\right)^{3 / 2}\right]\right\}^{1 / 2}} Q_{2}^{1} .
\end{gathered}
$$

\footnotetext{
${ }^{4}$ There is a misprint in equation (53) in [21], equation (3.96) here. The numerator of the second term to the right should be $\left(1-y_{1}^{2}\right)^{3 / 2}$.
} 
At the boundary of the configuration $r=R$, which is equivalent to $x_{R}=1-y_{1}$, the numerical solutions of the interior equations (3.61), (3.65), (3.71), (3.38) and (3.37), must match with the exterior solutions (3.92)-(3.96). Thus we have

$$
\begin{gathered}
\varpi(R)=\Omega-\frac{2 J}{\left(1-y_{1}^{2}\right)^{3 / 2}}, \\
m_{0}(R)=\delta M-\frac{J^{2}}{\left(1-y_{1}^{2}\right)^{3 / 2}}, \\
h_{0}(R)=-\frac{m_{0}(R)}{y_{1}^{2}\left(1-y_{1}^{2}\right)^{1 / 2}}, \\
h_{2}(R)=\frac{3-y_{1}^{2}}{\left(1-y_{1}^{2}\right)^{3}} J^{2}+K Q_{2}^{2}\left(\frac{1+y_{1}^{2}}{1-y_{1}^{2}}\right), \\
v_{2}(R)=-\frac{J^{2}}{\left(1-y_{1}^{2}\right)^{2}}+K \frac{\left(1-y_{1}^{2}\right)}{y_{1}} Q_{2}^{1}\left(\frac{1+y_{1}^{2}}{1-y_{1}^{2}}\right) .
\end{gathered}
$$

In summary:

- The particular solutions $h_{2}^{(p)}$ and $v_{2}^{(p)}$ will be found by integration of (3.74) and (3.75) with the origin behaviors given by (3.78) and (3.79), with the constant $a$ being assigned arbitrarily.

- The complementary functions $h_{2}^{(c)}$ and $v_{2}^{(c)}$ will be found by integrating equations (3.84) and (3.85) with the near origin behaviors given given by (3.86). The constant $B$ is designated arbitrarily.

- The constant $A$ in (3.83) and the constant $K$ in the exterior solutions (3.95) and (3.96), will be found by matching the interior and exterior solutions at the boundary of the star $r=R$. 


\subsubsection{ISOBARIC SURFACES}

Finally we consider the equation (3.35) for the isobaric surfaces. In terms of the variable $x$, the deformations $\xi_{0}$ and $\xi_{2}$, given by (3.36), take the forms

$$
\xi_{0}=\frac{(1-x)(k+x)}{[x(2-x)]^{1 / 2}} \delta P_{0}, \quad \xi_{2}=\frac{(1-x)(k+x)}{[x(2-x)]^{1 / 2}} \delta P_{2}
$$

Here we are measuring $\xi_{0}$ and $\xi_{2}$ in the unit $\alpha^{3} \varpi_{c}^{2}$. Using (3.44) the equation for the deformation $\xi_{2}$ can be recast in the form

$$
\xi_{2}=\frac{(1-x)(k+x)}{[x(2-x)]^{1 / 2}}\left[h_{2}+\frac{4 x(2-x)}{3(k+x)^{2}} \varpi^{2}\right]
$$

Using (3.98), the ellipticity of the spheroid (3.46) as a function of $x$ reads

$$
\varepsilon=\frac{3(1-x)(k+x)}{2 x(2-x)}\left[h_{2}+\frac{4 x(2-x)}{3(k+x)^{2}} \varpi(R)^{2}\right]-\frac{3}{2}\left(v_{2}-h_{2}\right),
$$

where $\varepsilon$ is being measured in the unit $\alpha^{2} \varpi_{c}^{2}$.

\subsubsection{Discussion}

We have assembled the equations to calculate the equilibrium structures of slowly rotating relativistic compact objects. In particular, we have discussed equations that relate mass and central density, and changes in the shape of the surface. These equations can be used to calculate surface and integral properties like moment of inertia, mass quadrupole moment and ellipticity. In the next chapter, we discuss the results of computations of equilibrium configurations of slowly rotating Schwarzschild stars, in particular in the ultracompact limit when the radius of the star approaches the Schwarzschild radius. 


\section{Chapter 4}

\section{Results}

What is now proved

was only once imagined

William Blake

\section{INTRODUCTION}

In chapter 2 we discussed the gravitational vacuum condensate stars, or gravastars, proposed by Mazur \& Mottola as an alternative to black holes for the final state of complete gravitational collapse. In connection with gravastars, we discussed the Schwarzschild interior solution, or Schwarzschild star, corresponding to the solution to Einstein's equations for a spherical perfect fluid with constant energy density $\epsilon$. We found that this solution shows a divergence in pressure when the radius of the star $R_{B}=(9 / 8) R_{S}$, known as the Schwarzschild-Buchdahl bound. The existence of this limit has been a motive of disregard of the interior solution as a 'realistic' physical description of a star.

In a bold approach, Mazur \& Mottola showed that the divergence in pressure is integrable through the Komar formula and a solution with negative pressure appears when $R<(9 / 8) R_{S}$. Moreover, in the ultracompact limit $R=R_{S}$, the Schwarzschild star becomes the gravitational condensate star with an interior governed by a modified patch of de Sitter spacetime with negative pressure $p=-\epsilon$, and a finite surface tension. The exterior remains the vacuum Schwarzschild spacetime. 
In chapter 3 we reviewed the method presented by Hartle to calculate equilibrium configurations of slowly rotating relativistic stars within the framework of perturbation theory. Chandrasekhar \& Miller used these equations of structure, to calculate surface and integral properties of a slowly rotating homogeneous mass with a constant energy density (Schwarzschild star). These computations were carried out numerically up to the Schwarzschild-Buchdahl bound.

Motivated by these developments, in this final chapter we present results of surface and integral properties for a slowly rotating Schwarzschild star, above the Buchdahl bound, and in the unstudied regime $R_{S}<R<(9 / 8) R_{S}$. An important result of this investigation is that in the gravastar limit $R \rightarrow R_{S}$, the surface properties approach to the corresponding Kerr values. These results, which have been accepted for publication [82], provide a long-sought model of a slowly rotating gravastar. Furthermore, these results have important implications for the old problem in general relativity and relativistic astrophysics, which is finding the source of a rotating Kerr black hole.

The chapter is organized as follows. In the next section, we present integral and surface properties for the Schwarzschild star with $R>R_{B}$. In section 4.2 we extend the analysis for models with $R_{S}<R<(9 / 8) R_{S}$. The integrations are listed in Tables C.1 and C.2 (see Appendix C). In section 4.3 we close the chapter with a final discussion.

\subsection{Surface And integral Properties of SchWarzschild Stars For $R>(9 / 8) R_{S}$}

In this section we present the integrations of the equations of structure for a homogeneous star with constant energy density $\epsilon$, when its radius is above the Buchdahl bound $R \geq(9 / 8) R_{S}$. The high complexity of the set of equations demanded the use of numerical and computational methods. In particular, we chose the adaptive Runge-Kutta-Fehlberg method [83]. It is well known that adaptive methods offer many 
advantages over the standard Runge-Kutta methods. In many situations a convenient choice of the integration step size $h$ can be a challenge. If $h$ is too large, we might obtain huge truncation errors; if $h$ is too small we might be misusing computational resources. The problem gets worse when the solutions presents stiff behavior near some value. In those cases, setting a constant step size might not be appropriate for the whole integration region.

In our particular case, the equations of structure blow up at the Buchdahl bound $R=(9 / 8) R_{S}$. Thus, when we approach this limit during the integrations, we might need to reduce the value of the step size. The adaptive methods provide a powerful technique to estimate and adjust the step size $h$ to maintain the truncation error within prescribed limits. In Appendix D we provide more details on these methods and some general formulae.

We used the programming language called $\mathrm{P}_{\text {YTHON }}{ }^{1}$, in particular we used the version 3.4 for UBUntu 14.04. PYTHON is a powerful and versatile language which is being used widely in many applications in Physics. Additionally, PYTHON is free and easy to learn, which makes it a very convenient choice in computational physics. We worked with the development environment called IDLE (Integrated Development Environment) in its version 3.4. The routines that we incorporated in our code have been, for the most part, adapted from the ones found in the standard textbooks on numerical and computational methods (see e.g. [49, 70, 83]).

The results of the integrations are listed in Table C.1 (see Appendix C). We followed the conventions found in [21] where dimensionless variables are being used and the corresponding units of the surface properties are specified in the caption. We simulated several models by varying the compactness parameter $R / R_{S}$, where $R$ is the radius of the star and $R_{S}$ is the Schwarzschild radius. The results are presented in a way which resembles an adiabatic and quasi-stationary contraction of the star [65].

\footnotetext{
${ }^{1}$ http: //python.org
} 


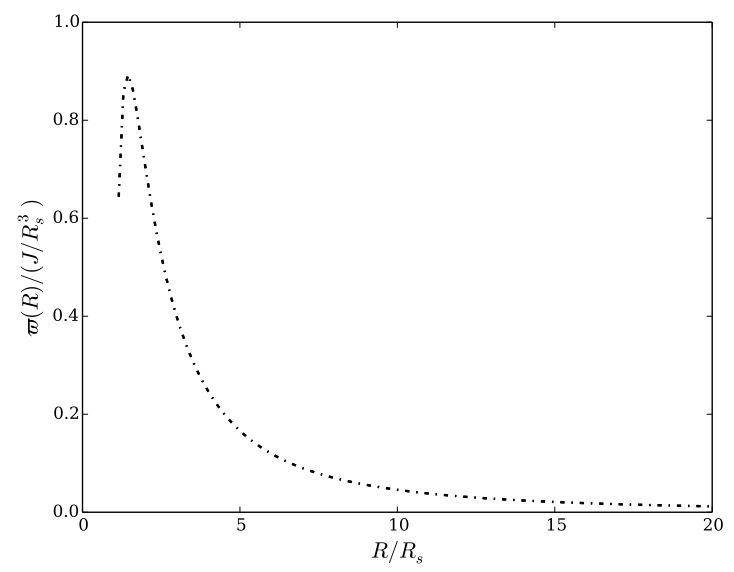

Figure 4.1: The angular velocity $\varpi=\left.(\Omega-\omega)\right|_{r=R}$ (in units of $J / R_{s}^{3}$ ) relative to the local ZAMO, plotted as a function of the compactness parameter $R / R_{s}$ above the Schwarzschild-Buchdahl bound $R>1.125 R_{s}$.

We reproduced the results published by Chandrasekhar \& Miller [21] for stellar models with $R>(9 / 8) R_{S}$ with agreement up to the fourth decimal place. However we corrected the value of the mass quadrupole moment $Q$ at the Buchdahl bound $R=1.125 R_{S}$, where we found $Q=2.02311$ (in units of $J^{2} / R_{S}$ ), in contrast to the value $Q=2.002$ published in [21]. The difference in this value might be associated to the old methods used in that paper, which dates back to 1974. Moreover, as it was mentioned above, the stiff behavior of the structure equations at the Buchdahl radius demands the use of adaptive methods, which is not clear if it was done in [21]. In the following we provide some illustrations of the main results of the integrations and further analysis.

In Fig. 4.1 we plotted the surface value of the fluid angular velocity $\varpi(R)$ relative to the local ZAMO, versus the compactness parameter $R / R_{s}$, above the SchwarzschildBuchdahl bound. Notice that $\varpi(R)$ reaches a maximum near to $R=1.4 R_{s}$ and then approaches zero in the Newtonian limit $R \rightarrow \infty$. These results are in very good agreement with [21].

In Fig. 4.2 the angular velocity $\Omega$ relative to a distant observer is plotted as a function of the compactness parameter. Note that $\Omega$ approaches the value 2 (in units 


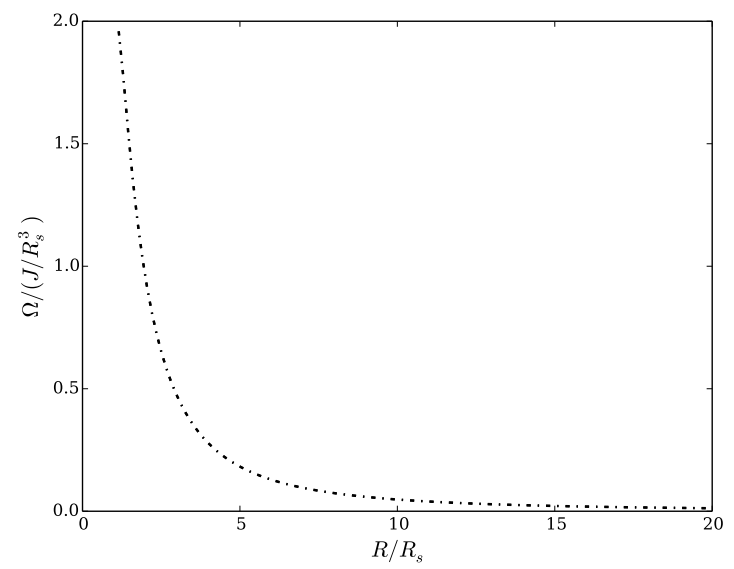

Figure 4.2: The angular velocity $\Omega$ (in units of $J / R_{s}^{3}$ ) relative to an observer at infinity, plotted as a function of the compactness parameter $R / R_{s}$ above the Buchdahl bound.

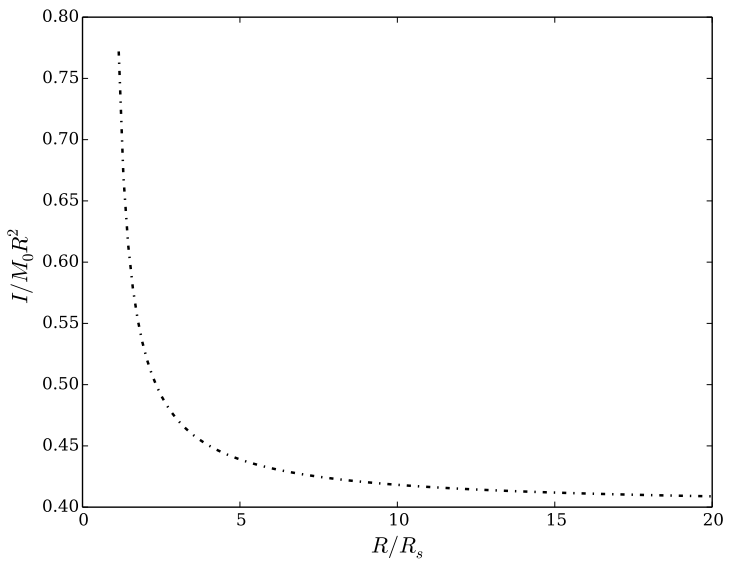

Figure 4.3: The normalized moment of inertia $I_{N}$ plotted as a function of the compactness parameter $R / R_{s}$ above the Buchdahl bound.

of $J / R_{s}^{3}$ ) when $R \rightarrow 1.125 R_{S}$, and tends to zero in the Newtonian limit. These results makes sense considering that, an increase in the size of the star increases its moment of inertia. Therefore, given that the angular momentum is conserved, the angular velocity must decrease.

The 'normalized' moment of inertia $I_{N}=I / M_{0} R^{2}$ as a function of $R / R_{S}$ is illustrated in figure 4.3. Note the approach of $I_{N}$ to the value 0.8 when $R$ approaches the Schwarzschild-Buchdahl bound. On the other hand, in the limit where $R \rightarrow \infty$ the moment of inertia approaches the value 0.4 , which corresponds to the moment of 


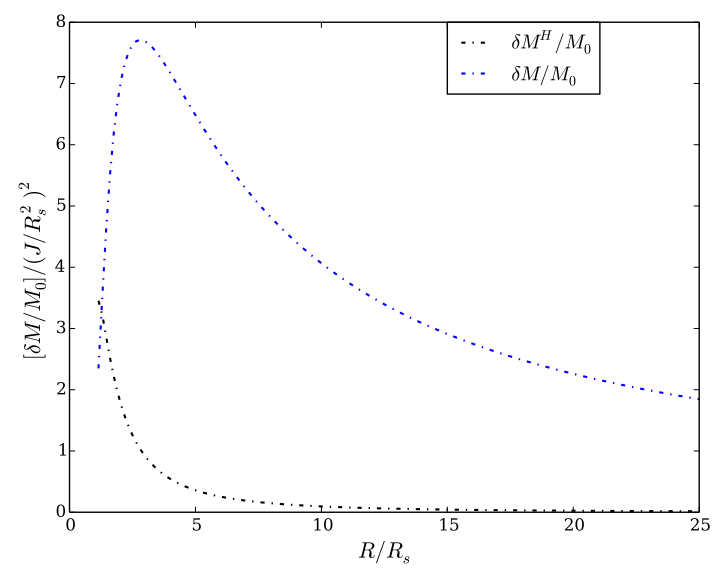

Figure 4.4: The original $\delta M^{H} / M_{0}$ and amended $\delta M / M_{0}$ fractional change of mass against the compactness parameter $R / R_{s}$ above the Buchdahl bound.

inertia of a sphere in the Newtonian regime. Let's recall that the first-order perturbations do not change the shape of the star, thus it is valid to compare with a sphere in this limit. Our results are in excellent agreement with [21].

In figure 4.4 are illustrated the, original and amended, fractional change in mass given by (3.33) and (3.34), as a function of $R / R_{S}$. Note that $\delta M / M$ reaches a maximum at $R \approx 2.8 R_{S}$ and then decreases when $R \rightarrow \infty$. Note also that the original Hartle's change of mass $\delta M^{H} / M$ decreases faster than the amended change of mass of Reina and Vera $[84,85]$. It is noteworthy to remark that the amended change of mass is not negligible for Schwarzschild stars with $R>(9 / 8) R_{S}$, moreover it's dominant. Our results are in very good agreement with [84].

Figures 4.5 and 4.6 show the behavior of the surface deformation functions $\xi_{0}$ and $\xi_{2}$ for a slowly rotating Schwarzschild star. In figures 4.7 and 4.8 we have plotted the ellipticity, as given by (3.99), as a function of the compactness parameter, for a Schwarzschild star with fixed total mass and angular momentum. Note that the ellipticity shows a non-monotonic behavior, reaching a maximum at $R / R_{S} \sim 2.4$. In principle, under adiabatic contraction, the star would become flatter due to the rotation. However, note that for $R<2.4 R_{S}$, there is a startling decrease in the 


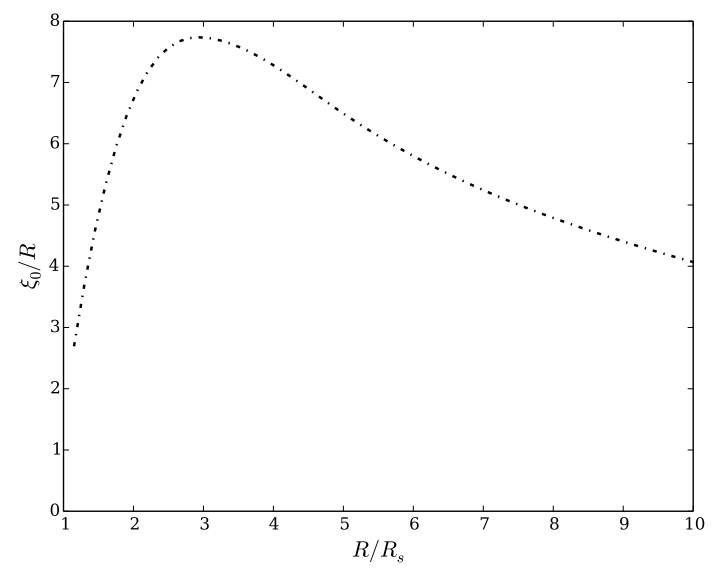

Figure 4.5: The deformation of the star $l=0$ : plot of $\xi_{0} / R$ (measured in units of $\left.J^{2} / R_{S}^{4}\right)$ as a function of the compactness $R / R_{S}$, for $R>(9 / 8) R_{S}$.

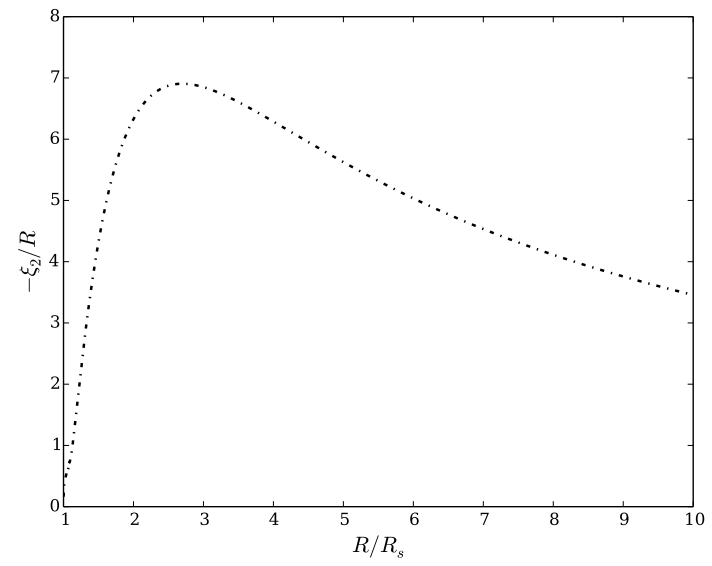

Figure 4.6: The deformation of the star $l=2$ : plot of $-\xi_{2} / R$ (measured in units of $\left.J^{2} / R_{S}^{4}\right)$ as a function of the compactness $R / R_{S}$, for $R>(9 / 8) R_{S}$.

ellipticity indicating that the star becomes more spherical. This behavior is contrary to what is expected for a Newtonian Maclaurin spheroid which, in the limit of small radius, takes the shape of an infinitesimal thin disc given by [18]

$$
\bar{\varepsilon}=\frac{125}{32} \frac{1}{R}
$$

where $R$ is measured in units of $R_{S}$. This reversal in behavior of the ellipticity, as the object contracts keeping its mass and angular momentum, has been the subject of lively discussion in the literature. Chandrasekhar \& Miller [21] argued that the 


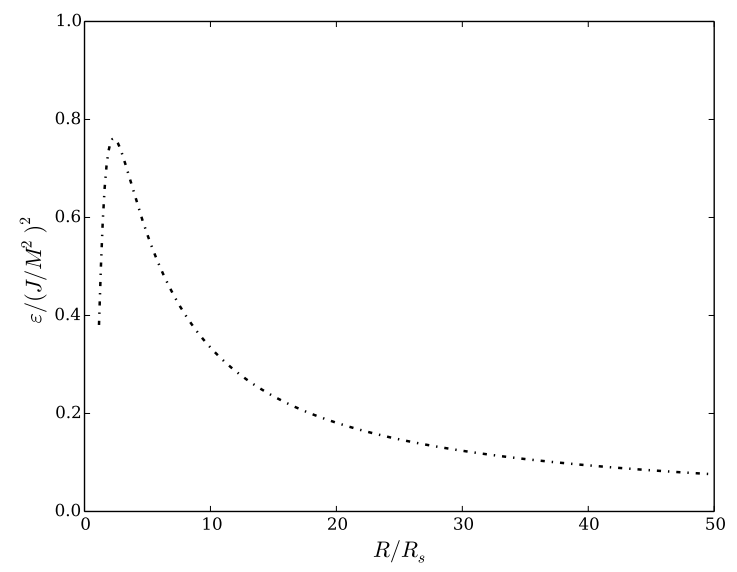

Figure 4.7: The ellipticity of the bounding surface (in units of $J^{2} / M^{4}$ ) as a function of the compactness parameter $R / R_{s}$ above the Buchdahl bound.

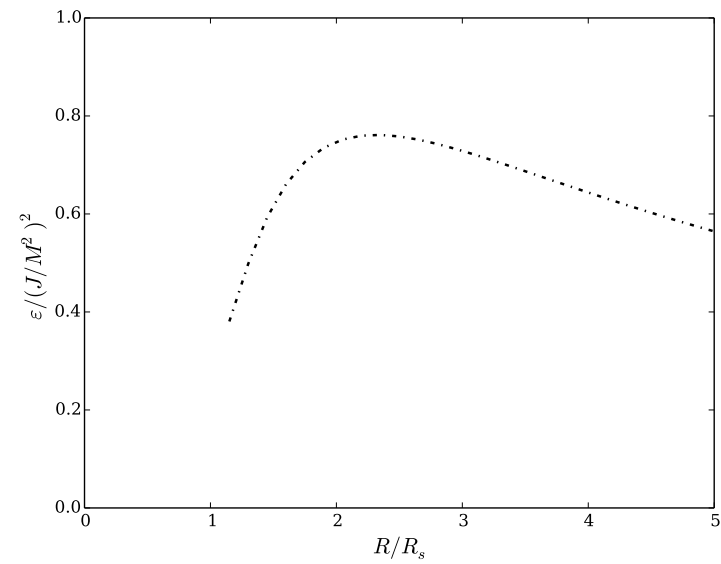

Figure 4.8: The ellipticity of the bounding surface (in units of $J^{2} / M^{4}$ ) as a function of the compactness parameter $R / R_{s}$ above the Buchdahl limit. The horizontal axis has been plotted with higher resolution to show more detail. 
centrifugal effects are due, not to the angular velocity $\Omega$ relative to a distant observer, but to the angular velocity $\varpi$ relative to the local ZAMO. Note that $\varpi$ decreases when $R / R_{S}<1.4$ (see Fig. 4.1) thus the second order terms in (3.99) are negligible. The fact that the maximum of the ellipticity does not occur at $R / R_{S} \sim 1.4$, in contrast to $\varpi$, can be explained by the dependence of $\varepsilon$ of the second order functions $h_{2}$ and $v_{2}$

On the other hand, Miller [65] argued that during contraction the increase in $\Omega$ is slower, compared to Newtonian theory, on account of an increase in the normalized moment of inertia. In all parts of the star $\varpi$ decreases monotonically, thus the centrifugal effects are weakened compared to what is expected in the Newtonian regime. In contrast to this explanation, Abramowicz \& Miller [6] suggested that the reversal in behavior of the ellipticity can be due to the peculiar effect of the reversal of the centrifugal force in strong gravitational fields $[2,3]$. Using a Newtonian approximation, Chakrabarti and Khanna [18] suggested that the reversal of the ellipticity, which according to them occurs at later stages compared to $R / R_{S} \sim 2.4$, is due to the self-coupling of the angular momentum of the star.

Here we agree with the explanation given in [21]. Equation (3.99) shows clearly that the ellipticity depends not only on the centrifugal effects as described by $\varpi$, but also of the second-order functions $h_{2}$ and $v_{2}$ which might have complicated behaviors. Finally, it is important to remark that the relativistic expression for the ellipticity is much more complicated than the Newtonian relation (4.1), therefore a different behavior from what is expected in the Newtonian case is not that surprising at all.

Finally in figure 4.9 we plot the 'Kerr' factor $Q M_{0} / J^{2}$ against the compactness $R / R_{S}$. Notice the linear behavior of the Kerr factor and its subsequent approach to the value 1 in the limit $R / R_{S} \rightarrow 1.125$. These results agrees with those in [65] and [101]. In their original paper Chandrasekhar \& Miller [21] found that the quadrupole moment, in units of $J^{2} / R_{s}$, is $Q=2.002$ at the Buchdahl radius $R=(9 / 8) R_{S}$. 


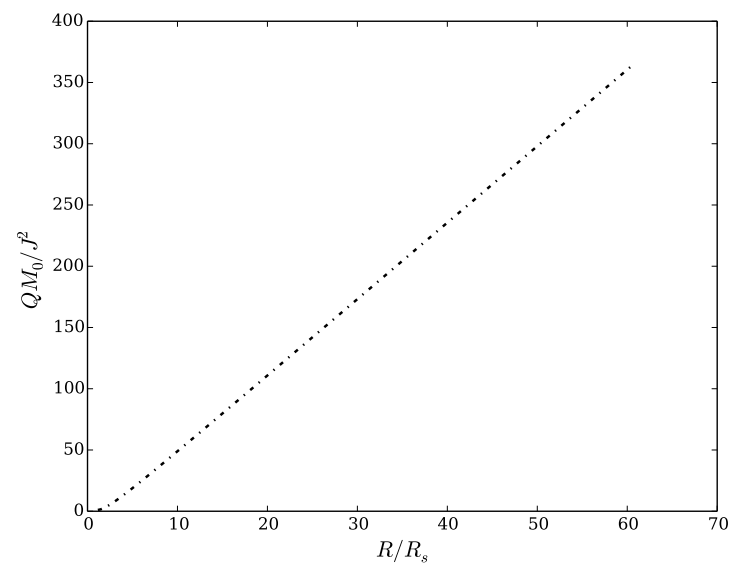

Figure 4.9: Plot of the 'Kerr' factor $Q M_{0} / J^{2}$ as a function of the compactness parameter $R / R_{s}$, for $R$ above the Schwarzschild-Buchdahl limit.

Motivated by this result they concluded the following

"Consequently, the metric external to a slowly rotating configuration of minimum radius agrees with the Kerr metric to a requisite order to one part in a thousand."

We revisited this value and we found $Q=2.0231$ (see table C.2), which challenges their conclusion. Moreover we will see in the next section, when we study Schwarzschild stars with $R_{S}<R<(9 / 8) R_{S}$, that the challenge is deeper considering that the gravastar is the limit configuration of an ultra-compact Schwarzschild star with minimum radius $R=R_{S}$.

\subsection{Surface AND Integral Properties of SCHWARZSChild STARS IN The REGIME $R_{S}<R<(9 / 8) R_{S}$}

In this section we present results of surface and integral properties for a slowly rotating Schwarzschild star, in the regime $R_{S}<R<(9 / 8) R_{S}$. We used the same methods which were described in the last section. The results of the numerical integrations are listed in Table C.2. 
Although the equations of structure for a Schwarzschild star, discussed in section 3.5 , preserve the same form for models with $R<(9 / 8) R_{S}$, there is an important change which now we discuss. Notice that the quantity $\left(3 y_{1}-y\right)$, which appears in the interior solution (3.54)-(3.56), becomes negative when $R<(9 / 8) R_{S}$. However the metric element $e^{2 \nu_{0}}$ in (2.35) is a perfect square, therefore it is always a positive quantity. Thus, in order to investigate the region $R_{s}<R<(9 / 8) R_{s}$, it is crucial to specify the modulus condition $\left|3 y_{1}-y\right|$. With these considerations, the Schwarzschild interior solution takes the form

$$
\begin{gathered}
\mathrm{e}^{\lambda_{0}}=\frac{1}{y}, \quad \mathrm{e}^{\nu_{0}}=\frac{1}{2}\left|3 y_{1}-y\right|, \\
\frac{p}{\epsilon}=\frac{y-y_{1}}{3 y_{1}-y}, \\
j=\frac{2 y}{\left|3 y_{1}-y\right|}, \quad y^{2}=1-\frac{2 m(r)}{r} .
\end{gathered}
$$

These changes were taken into account in our routine to compute the numerical solutions. Additionally we considered the modulus of the parameter $k(3.57)$

$$
k=\left|3 y_{1}-1\right|,
$$

which must also be positive in the regime $R_{S}<R<(9 / 8) R_{S}$. We will find convenient to introduce the 'Schwarzschild deviation parameter'

$$
\zeta \equiv \frac{R-R_{S}}{R_{S}},
$$

together with the quantity [12]

$$
\frac{\Delta Q}{Q} \equiv \frac{Q-Q_{K e r r}}{Q_{K e r r}}
$$




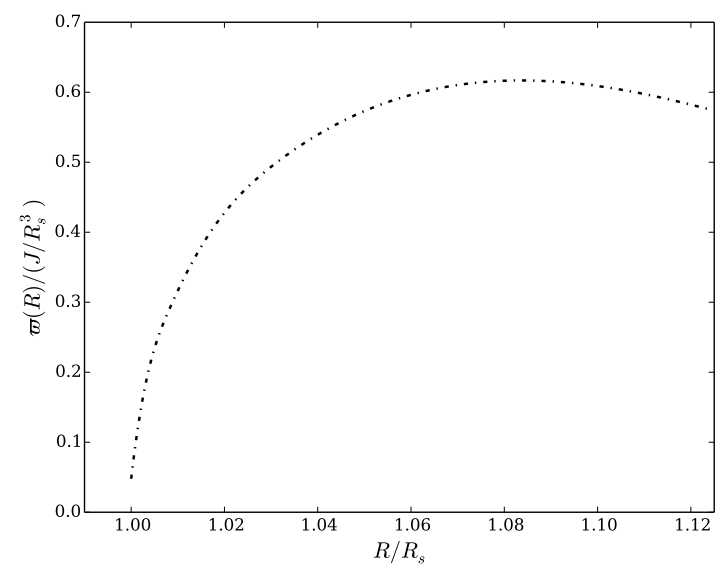

Figure 4.10: The angular velocity $\varpi=\left.(\Omega-\omega)\right|_{r=R}$ (in units of $J / R_{s}^{3}$ ) relative to the local ZAMO, plotted as a function of the compactness parameter $R / R_{s}$ in the regime $R_{S}<R<(9 / 8) R_{S}$

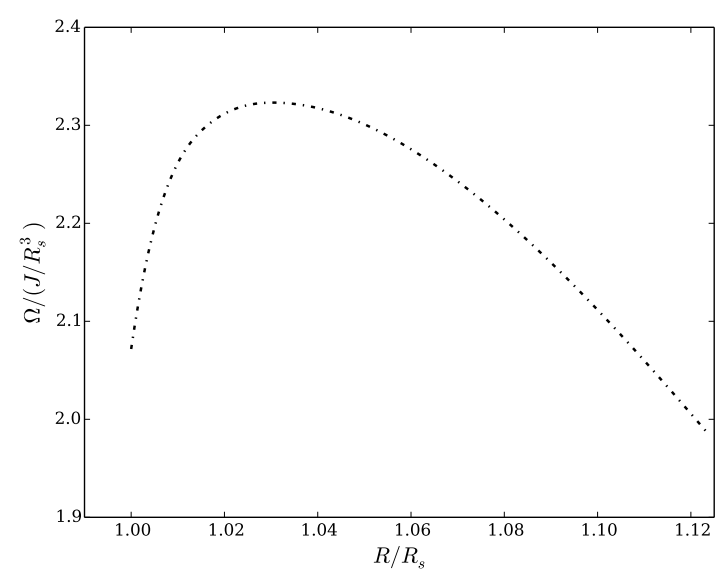

Figure 4.11: The angular velocity $\Omega(R)$ (in units of $J / R_{s}^{3}$ ) relative to a distant observer, plotted as a function of the compactness parameter $R / R_{s}$ in the regime $R_{S}<R<(9 / 8) R_{S}$

which denotes relative deviations of the mass quadrupole moment from the Kerr value $Q_{K e r r}=J^{2} / M_{0}$. In the following we discuss our results.

In figure 4.10 we plot the angular velocity $\varpi(R)$, relative to the local ZAMO, as a function of the compactness parameter $R / R_{S}$, for models with $R_{S}<R<(9 / 8) R_{S}$. Note that in the gravastar limit, when $R \rightarrow R_{S}, \varpi(R)$ goes to zero. In connection with this result, in figure 4.11 we show the behavior of the angular velocity $\Omega(R)$, as measured at infinity, as a function of $R / R_{S}$ for radii below the Buchdahl limit. Note 
the increase in $\Omega(R)$ up to a maximum value near $R / R_{S} \approx 1.03$, and the subsequent decrease towards the value 2 (in units of $J / R_{S}^{3}$ ) when $R \rightarrow R_{S}^{+}$. Furthermore, as figure 4.10 shows, in this limit $\varpi \rightarrow 0$ thus the angular velocity $\Omega=\omega$ is a constant indicating a rigidly rotating compact object with no differential surface rotation [57].

We now show that the value $\omega=\Omega=2$ (in units of $J / R_{s}^{3}$ ) for the angular velocity of the ultra-compact Schwarzschild star in the gravastar limit $\left(\zeta \sim 10^{-14}\right)$ is consistent with that of the Kerr black hole limit. It is well known that in the Kerr spacetime, a radially falling test particle with zero angular momentum acquires an angular velocity when it approaches the spinning black hole (see section 1.4). The angular velocity as measured by a distant ZAMO is given by

$$
\omega=\frac{d \phi}{d t}=\frac{2 a M_{0} r}{\left(r^{2}+a^{2}\right)^{2}-\Delta(r) a^{2} \sin ^{2} \theta},
$$

where $a \equiv J / M_{0}$ and $\Delta(r) \equiv r^{2}-2 M_{0} r+a^{2}$. Notice that positive $a$ implies positive $\omega$, therefore the particle will rotate in the spinning direction of the black hole. This is the so-called dragging effect in Kerr geometry which was discussed in section 1.4. At the 'event horizon' the following conditions are satisfied

$$
\Delta=0, \quad r=r_{+}=M_{0}+\left(M_{0}^{2}-a^{2}\right)^{1 / 2},
$$

where $r_{+}$was defined in (1.46). Using the condition (4.9) into (4.8) we have

$$
\omega_{b h}=\frac{a}{2 M_{0} r_{+}}
$$

which corresponds to the angular velocity of the Kerr black hole. In the slowly rotating approximation $\left(\xi \equiv a / M_{0}<<1\right)$ a straightforward calculation from (4.10) shows that

$$
\Omega=\omega_{b h} \approx \frac{a}{4 M_{0}^{2}}+\mathcal{O}\left(\xi^{2}\right)=2\left(\frac{J}{R_{s^{3}}}\right)+\mathcal{O}\left(\xi^{2}\right)
$$




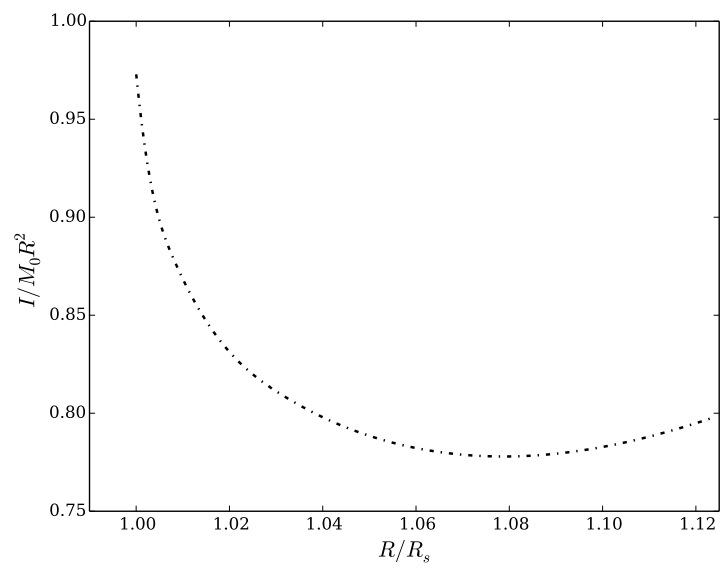

Figure 4.12: The normalized moment of inertia $I_{N} \equiv I / M_{0} R^{2}$ plotted as a function of the compactness $R / R_{s}$, for the Schwarzschild star in the regime $R_{S}<R<(9 / 8) R_{S}$. Notice the approach of $I_{N}$ to 1 in the gravastar limit $R \rightarrow R_{S}^{+}$.

which is consistent with our numerical results for $\Omega$ in the gravastar limit $\zeta \sim 10^{-14}$ (see Table C.1).

In figure 4.12 we plot the normalized moment of inertia $I_{N} \equiv I / M_{0} R^{2}$ against the compactness $R / R_{S}$. We notice that, during contraction $R^{+} \rightarrow R_{S}, I_{N}$ decreases slowly reaching a minimum value at $R / R_{S} \sim 1.08$. Afterwards $I_{N}$ increases approaching the value 1 in the gravastar limit $R \rightarrow R_{S}$. This result is in remarkable consistency with the Kerr black hole value, in the slowly rotating approximation, which corresponds to [74]

$$
I=\frac{J}{\omega_{b h}} \approx 4 M_{0}^{3}+O\left(\xi^{2}\right)
$$

Figure 4.13 shows the original $\delta M^{H} / M$ and amended $\delta M / M$ fractional change in mass as a function of the parameter $R / R_{s}$, in the regime $R_{s}<R<(9 / 8) R_{s}$. Notice that $\delta M^{H}$ decreases more rapidly than the amended $\delta M$, however both of them approach to the value 2 (in units of $J^{2} / R_{S}^{4}$ ) in the gravastar limit $R^{+} \rightarrow R_{s}$. The fact that the correction term is negligible at the gravastar limit, can be understood by inspection of the relation for the amended change of mass (3.34). Even though a 


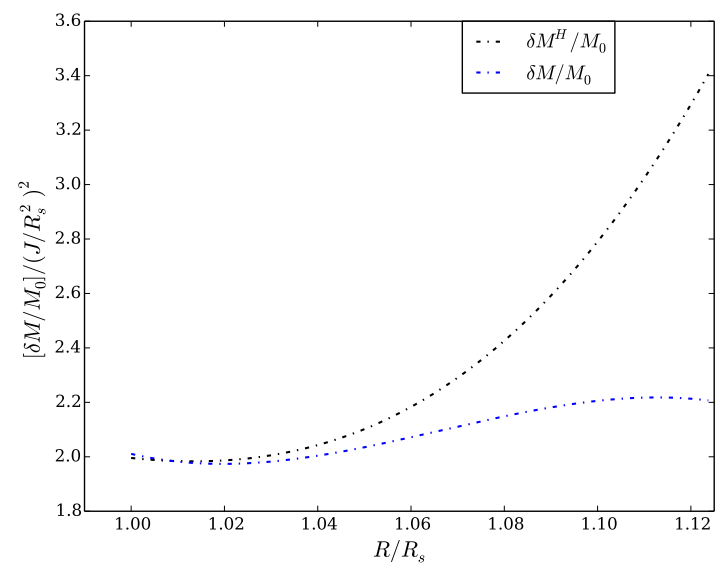

Figure 4.13: The original and amended $\delta M$ change of mass as a function of the compactness $R / R_{s}$, for Schwarzschild stars in the regime $R_{S}<R<(9 / 8) R_{S}$.

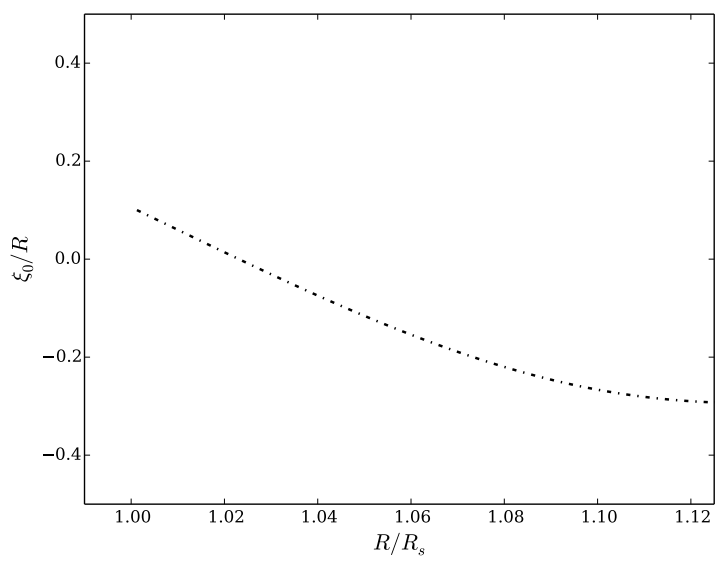

Figure 4.14: The deformation of the star $l=0$ : plot of $\xi_{0} / R$ (measured in units of $\left.J^{2} / R_{S}^{4}\right)$ as a function of the compactness $R / R_{S}$, for $R_{S}<R<(9 / 8) R_{S}$.

gravastar has a finite surface energy (associated to the surface tension) at $R=2 M_{0}$, the factor $\left(R-2 M_{0}\right)$ vanishes there.

Figures 4.14, 4.15 and 4.16 illustrate the deformations of the bounding surface, as described by the functions $\xi_{0}(R),-\xi_{2}(R)$ and $\varepsilon(R)$, for a Schwarzschild star with constant mass and angular momentum. Notice the monotonic behavior of the $l=0$ and $l=2$ deformations. Regarding the ellipticity $\varepsilon(R)$, notice how it decreases monotonically as the star contracts and subsequently approaches to the value 0.375 (in units of $J^{2} / M^{4}$ ) in the gravastar limit $R^{+} \rightarrow R_{S}$ (see Table C.2). 


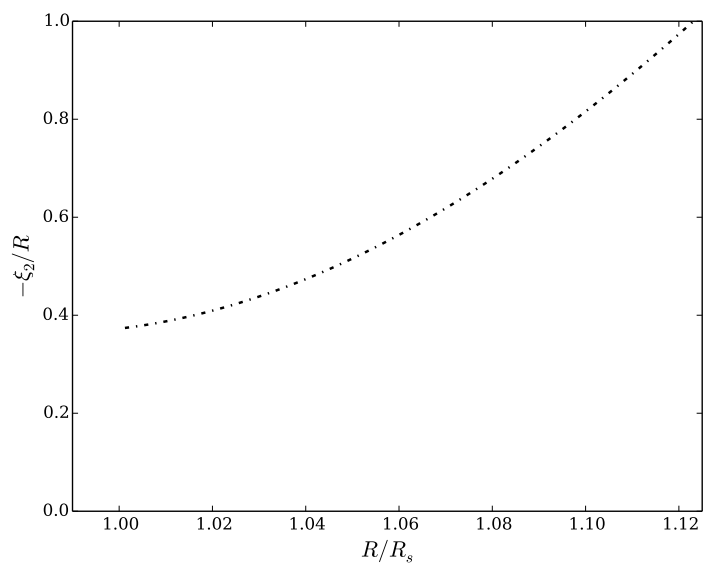

Figure 4.15: The deformation of the star $l=2$ : plot of $-\xi_{2} / R$ (measured in units of $\left.J^{2} / R_{S}^{4}\right)$ as a function of the compactness $R / R_{S}$, for $R_{S}<R<(9 / 8) R_{S}$.

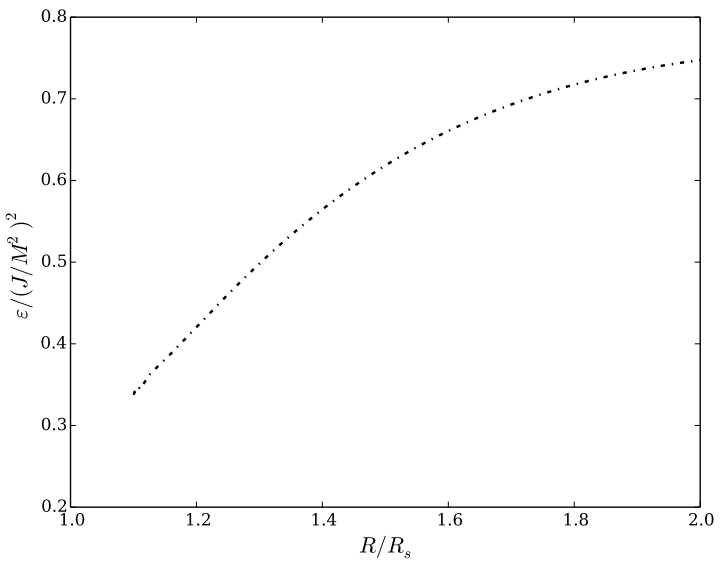

Figure 4.16: The ellipticity of the bounding surface (in units of $J^{2} / M^{4}$ ) as a function of the compactness parameter $R / R_{s}$, in the regime $R_{S}<R<(9 / 8) R_{S}$.

Finally, in figure 4.17 the Kerr factor $\bar{q}=Q M_{0} / J^{2}[65,100,101]$, is plotted against the compactness $R / R_{S}$. Notice that in the gravastar limit $R^{+} \rightarrow R_{S}$ the Kerr factor approaches to 1, which corresponds to the Kerr metric value. A remarkable result is that relative deviations of the mass quadrupole moment, as given by (4.7), are of the order of $10^{-15}$ in the gravastar limit $R^{+} \rightarrow R_{S}$ with deviations of the order $\zeta \sim 10^{-14}$. Thus, we conclude that the exterior metric to a slowly rotating ultracompact Schwarzschild star in the gravastar limit, with interior negative pressure, agrees to an accuracy of 1 part in $10^{15}$ with the Kerr metric in the slowly rotating 


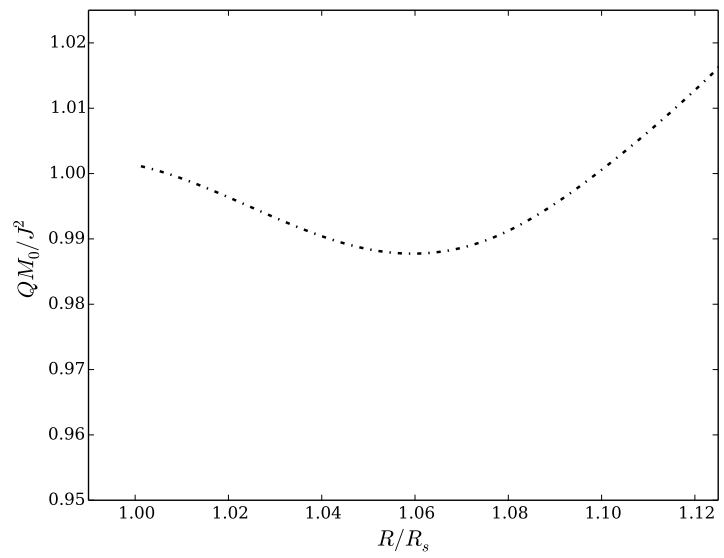

Figure 4.17: Plot of the 'Kerr' factor $Q M_{0} / J^{2}$ as a function of the compactness parameter $R / R_{s}$, for $R_{S}<R<(9 / 8) R_{S}$. Notice the approach to the value 1 , when $R^{+} \rightarrow R_{S}$

approximation.

\subsection{Discussion}

Motivated by recent investigations of [63] and the methods introduced by [33] and [21] in the study of slowly rotating relativistic masses, we have presented in this paper results for integral and surface properties of a slowly rotating super-compact Schwarzschild star in the unstudied regime $R_{s}<R<(9 / 8) R_{s}$. We found that the angular velocity $\varpi$ relative to the local ZAMO tends to zero in the gravastar limit $R \rightarrow R_{s}^{+}$. This result indicates that the super-compact Schwarzschild star rotates rigidly with no differential surface rotation. Furthermore the angular velocity $\Omega$ of the super-compact Schwarzschild star, in the gravastar limit, is constant and approaches the corresponding Kerr value in the slowly rotating approximation. Additionally, we found that the normalized moment of inertia $I / M_{0} R^{2}$ approaches 1 systematically when $R \rightarrow R_{s}^{+}$. This result is in agreement with the value corresponding to the slowly rotating Kerr metric. The most remarkable result concerns the mass quadrupole moment Q. We found that for a slowly rotating super-compact Schwarzschild star, in 
the gravastar limit, the relative deviation factor is $\Delta Q / Q \sim 10^{-15}$. These aforementioned results indicate that the external metric of a slowly rotating super-compact Schwarzschild star in the gravastar limit, agrees with the Kerr metric to the requisite order to one part in $10^{15}$. These results provide the long-sought solution to the problem of the source of rotation of the slowly rotating Kerr metric. 


\section{BIBLIOGRAPHY}

[1] B. P. Abbott et al., Observation of Gravitational Waves from a Binary Black Hole Merger, Phys. Rev. Lett. 116 (2016), no. 6, 061102.

[2] M. A. Abramowicz, Centrifugal Force - a Few Surprises, Mon. Not. Roy. Astron. Soc. 245 (1990), 733.

[3] _ Black holes and the centrifugal force paradox., Sci. Am. 268 (1993), $26-31$.

[4] M. A. Abramowicz, T. Bulik, G. F. R. Ellis, K. A. Meissner, and M. Wielgus, The electromagnetic afterglows of gravitational waves as a test for Quantum Gravity, 2016, arXiv: gr-gc/1603.07830.

[5] M. A. Abramowicz, W. Kluzniak, and J. P. Lasota, No observational proof of the black hole event-horizon, Astron. Astrophys. 396 (2002), L31-L34.

[6] M. A. Abramowicz and J. C. Miller, Ellipticity Behaviour of Relativistic Maclaurin Spheroids, Mon. Not. Roy. Astron. Soc. 245 (1990), 729.

[7] J. M. Bardeen, B. Carter, and S. W. Hawking, The Four laws of black hole mechanics, Commun. Math. Phys. 31 (1973), 161-170.

[8] J. D. Bekenstein, Black holes and entropy, Phys. Rev. D7 (1973), 2333-2346.

[9] Generalized second law of thermodynamics in black hole physics, Phys. Rev. D9 (1974), 3292-3300.

[10] V. Berezin, Black hole thermodynamics without a black hole?, Nucl. Phys. B661 (2003), 409-422.

[11] E. Bianchi and C. Rovelli, Why all these prejudices against a constant?, 2010, arXiv: 1002.3966 .

[12] M. Bradley and G. Fodor, The quadrupole moment of slowly rotating fluid balls, Phys. Rev. D79 (2009), 044018. 
[13] H. A. Buchdahl, General Relativistic Fluid Spheres, Phys. Rev. 116 (1959), 1027.

[14] S. M. Carroll, Spacetime and geometry: An introduction to general relativity, Addison Wesley, 2004.

[15] B. Carter, Axisymmetric Black Hole Has Only Two Degrees of Freedom, Phys. Rev. Lett. 26 (1971), 331-333.

[16] C. Cattoen, T. Faber, and M. Visser, Gravastars must have anisotropic pressures, Class. Quantum Grav. 22 (2005), 4189-4202.

[17] A. Celotti, J. C. Miller, and D. W. Sciama, Astrophysical evidence for the existence of black holes: Topical review, Class. Quantum Grav. 16 (1999), A3.

[18] S. K. Chakrabarti and R. Khanna, A Newtonian description of the geometry around a rotating black hole, Mon. Not. Roy. Astron. Soc. 256 (1992), 300-306.

[19] S. Chandrasekhar, The maximum mass of ideal white dwarfs, Astrophys. J. 74 (1931), 81-82.

[20] - The mathematical theory of black holes, Oxford University Press, 1992.

[21] S. Chandrasekhar and J. C. Miller, On slowly rotating homogeneous masses in general relativity, Mon. Not. Roy. Astron. Soc. 167 (1974), no. 1, 63-80.

[22] G. Chapline, E. Hohlfeld, R. B. Laughlin, and D. I. Santiago, Quantum phase transitions and the breakdown of classical general relativity, Int. J. Mod. Phys. A18 (2003), 3587-3590.

[23] C. Chirenti and L. Rezzolla, Did GW150914 produce a rotating gravastar?, Phys. Rev. D94 (2016), no. 8, 084016.

[24] C. B. M. H. Chirenti and L. Rezzolla, How to tell a gravastar from a black hole, Class. Quantum Grav. 24 (2007), 4191-4206.

[25] D. Christodoulou, Reversible and irreversible transforations in black hole physics, Phys. Rev. Lett. 25 (1970), 1596-1597.

[26] G. Dautcourt, Race for the Kerr field, Gen. Rel. Grav. 41 (2009), 1437-1454. 
[27] I. Dymnikova, Spherically symmetric space-time with the regular de Sitter center, Int. J. Mod. Phys. D12 (2003), 1015-1034.

[28] A. Einstein, H. A. Lorentz, H. Weyl, and H. Minkowski, The principle of relativity: A collection of original papers on the special and general theory of relativity., Dover, 1952.

[29] V. P. Frolov, Do Black Holes Exist?, Proceedings, 18th International Seminar on High Energy Physics (Quarks 2014): Suzdal, Russia, June 2-8, 2014, 2014.

[30] E. B. Gliner, Inflationary universe and the vacuumlike state of physical medium, Phys. Usp. 45 (2002), 213-220.

[31] T. Gold, Rotating neutron stars as the origin of the pulsating radio sources, Nature 218 (1968), 731-732.

[32] B. K. Harrison, K. S. Thorne, M. Wakano, and J. A. Wheeler, Gravitation Theory and Gravitational Collapse, University of Chicago Press, 1965.

[33] J. B. Hartle, Slowly rotating relativistic stars. I. Equations of structure, Astrophys. J. 150 (1967), 1005-1029.

[34] _ An introduction to Einstein's general relativity, Addison-Wesley, 2003.

[35] J. B. Hartle and D. H. Sharp, Variational Principle for the Equilibrium of a Relativistic, Rotating Star, Astrophys. J. 147 (1967), 317.

[36] J. B. Hartle and K. S. Thorne, Slowly Rotating Relativistic Stars. II. Models for Neutron Stars and Supermassive Stars, Astrophys. J. 153 (1968), 807.

[37] S. W. Hawking, Gravitational radiation from colliding black holes, Phys. Rev. Lett. 26 (1971), 1344-1346.

[38] , Particle Creation by Black Holes, Commun. Math. Phys. 43 (1975), 199-220, [,167(1975)].

[39] - Breakdown of Predictability in Gravitational Collapse, Phys. Rev. D14 (1976), 2460-2473.

[40] - Information Preservation and Weather Forecasting for Black Holes, 2014, arXiv: hep-th/1401.5761. 
[41] S. W. Hawking and G. F. R. Ellis, The large scale structure of space-time, Cambridge University Press, 1973.

[42] W. C. Hernandez, Material Sources for the Kerr Metric, Phys. Rev. 159 (1967), 1070-1072.

[43] A. Hewish, S. J. Bell, J. D. H Pilkington, P. F. Scott, and R. A. Collins, Observation of a rapidly pulsating radio source, Nature 217 (1968), 709-713.

[44] D. Horvat, S. Ilijic, and A. Marunovic, Electrically charged gravastar configurations, Class. Quantum Grav. 26 (2009), 025003.

[45] W. Israel, Event horizons in static vacuum space-times, Phys. Rev. 164 (1967), 1776-1779.

[46] Event horizons in static electrovac space-times, Commun. Math. Phys. 8 (1968), 245-260.

[47] S Source of the kerr metric, Phys. Rev. D2 (1970), 641-646, [Phys. Rev.D2,64L(1970)].

[48] R. P. Kerr, Gravitational field of a spinning mass as an example of algebraically special metrics, Phys. Rev. Lett. 11 (1963), 237-238.

[49] J. Kiusalaas, Numerical methods in engineering with python, 2nd. ed., Cambridge University Press, 2010.

[50] A. Komar, Covariant conservation laws in general relativity, Phys. Rev. 113 (1959), 934-936.

[51] M. D. Kruskal, Maximal extension of Schwarzschild metric, Phys. Rev. 119 (1960), 1743-1745.

[52] L. D. Landau and E. M. Lifshitz, The classical theory of fields, 2nd. ed., Pergamon Press, 1962.

[53] R. B. Laughlin, Emergent relativity, Int. J. Mod. Phys. A18 (2003), 831-854.

[54] F. S. N. Lobo, Stable dark energy stars, Class. Quantum Grav. 23 (2006), 15251541. 
[55] E. Mach, The science of mechanics: A critical and historical account of its development, Open court publishing Company, 1907.

[56] M. Mars, First and second order perturbations of hypersurfaces, Class. Quantum Grav. 22 (2005), 3325-3348.

[57] G. E. Marsh, Rigid Rotation and the Kerr Metric, 2014, arXiv: gr-qc/1404.5297.

[58] P. O. Mazur, Proof of uniqueness of the Kerr-Newman black hole solution, J. Phys. A15 (1982), 3173-3180.

[59] G Gravitation, the quantum, and cosmological constant, Acta Phys. Polon. 27 (1996), 1849-1858.

[60] P. O. Mazur and E. Mottola, Gravitational condensate stars: An alternative to black holes, 2001, arXiv: gr-qc/0109035.

[61] _ Dark energy and condensate stars: Casimir energy in the large, Quantum field theory under the influence of external conditions. Proceedings, 6th Workshop, QFEXT'03, Norman, USA, September 15-19, 2003, 2004, pp. 350357.

[62] _ Gravitational vacuum condensate stars, Proc. Nat. Acad. Sci. 101 (2004), 9545-9550.

[63]__ Surface tension and negative pressure interior of a non-singular 'black hole', Class. Quantum Grav. 32 (2015), no. 21, 215024.

[64] J. Miller and M. Colpi, Rotational properties of strange stars, Nucl. Phys. Proc. Suppl. 24B (1991), 166-169, [,166(1991)].

[65] J. C. Miller, Quasi-stationary gravitational collapse of slowly rotating bodies in general relativity, Mon. Not. Roy. Astron. Soc. 179 (1977), 483-498.

[66] C. W. Misner, K. S. Thorne, and J. A. Wheeler, Gravitation, W. H. Freeman, 1973.

[67] E. Mottola, New Horizons in Gravity: Dark Energy and Condensate Stars, J. Phys. Conf. Ser. 314 (2011), 012010.

[68] J. V. Narlikar, An Introduction to Relativity, Cambridge University Press, 2010. 
[69] E T. Newman, R. Couch, K. Chinnapared, A. Exton, A. Prakash, and R. Torrence, Metric of a Rotating, Charged Mass, J. Math. Phys. 6 (1965), 918-919.

[70] M. E. J. Newman, Computational Physics, Createspace, 2012.

[71] B. O'Neill, The Geometry of Kerr Black Holes, Dover, 2014.

[72] J. R. Oppenheimer and G. M. Volkoff, On Massive neutron cores, Phys. Rev. 55 (1939), 374-381.

[73] D. N. Page, Hawking radiation and black hole thermodynamics, New J. Phys. 7 (2005), 203.

[74] P. Pani, I-Love-Q relations for gravastars and the approach to the blackhole limit, Phys. Rev. D92 (2015), no. 12, 124030, [Erratum: Phys. Rev.D95,no.4,049902(2017)].

[75] P. Pani, E. Berti, V. Cardoso, Y. Chen, and R. Norte, Gravitational wave signatures of the absence of an event horizon. I. Nonradial oscillations of a thin-shell gravastar, Phys. Rev. D80 (2009), 124047.

[76] Gravitational-wave signatures of the absence of an event horizon. II. Extreme mass ratio inspirals in the spacetime of a thin-shell gravastar, Phys. Rev. D81 (2010), 084011.

[77] R. Penrose and R. M. Floyd, Extraction of rotational energy from a black hole, Nature 229 (1971), 177-179.

[78] S. Perlmutter et al., Measurements of Omega and Lambda from 42 high redshift supernovae, Astrophys. J. 517 (1999), 565-586.

[79] C. Pichon and Donald Lynden-Bell, New sources for kerr and other metrics: rotating relativistic disks with pressure support, Mon. Not. Roy. Astron. Soc. 280 (1996), 1007.

[80] J. Plebański and A. Krasiński, An introduction to general relativity and cosmology, Cambridge University Press, 2006.

[81] E. Poisson, A Relativist's Toolkit, Cambridge University Press, 2004. 
[82] C. Posada, Slowly rotating supercompact Schwarzschild stars, Mon. Not. Roy. Astron. Soc. 468 (2017), no. 2, 2128-2139.

[83] W. H. Press, S. A. Teukolsky, W. T. Vetterling, and B. P. Flannery, Numerical recipes in $C$. The art of scientific computing, 2nd. ed., Cambridge University Press, 1992.

[84] B. Reina, Slowly rotating homogeneous masses revisited, Mon. Not. Roy. Astron. Soc. 455 (2016), no. 4, 4512-4517.

[85] B. Reina and R. Vera, Revisiting Hartle's model using perturbed matching theory to second order: amending the change in mass, Class. Quantum Grav. 32 (2015), no. 15, 155008.

[86] L. Rezzolla and O. Zanotti, Relativistic Hydrodynamics, Oxford University Press, 2013.

[87] A. G. Riess et al., Observational evidence from supernovae for an accelerating universe and a cosmological constant, Astron. J. 116 (1998), 1009-1038.

[88] D. C. Robinson, Uniqueness of the Kerr black hole, Phys. Rev. Lett. 34 (1975), 905-906.

[89] R. Ruffini and J. A. Wheeler, Introducing the black hole, Phys. Today 24 (1971), no. 1,30 .

[90] B. F. Schutz, A first course in general relativity, Cambridge University Press, 1985.

[91] K. Schwarzschild, On the gravitational field of a mass point according to Einstein's theory, Sitzungsber. Preuss. Akad. Wiss. Berlin (Math. Phys.) 1916 (1916), 189-196.

[92] _ On the gravitational field of a sphere of incompressible fluid according to Einstein's theory, Sitzungsber. Preuss. Akad. Wiss. Berlin (Math. Phys.) 1916 (1916), 424-434.

[93] S. L. Shapiro and S. A. Teukolsky, Black holes, white dwarfs, and neutron stars: The physics of compact objects, Wiley, 1983. 
[94] L. Smarr, Mass formula for Kerr black holes, Phys. Rev. Lett. 30 (1973), 71-73, [Erratum: Phys. Rev. Lett.30,521(1973)].

[95] H. Stephani, Relativity : An introduction to special and general relativity, 3rd. ed., Cambridge University Press, 2004.

[96] C. R. Stephens, G. 't Hooft, and B. F. Whiting, Black hole evaporation without information loss, Class. Quantum Grav. 11 (1994), 621-648.

[97] E. Stoner, The Equilibrium of Dense Stars, Phil. Mag. 9 (1930), 944-963.

[98] G. Szekeres, On the singularities of a Riemannian manifold, Publ. Math. Debrecen 7 (1960), 285-301.

[99] S. A. Teukolsky, The Kerr Metric, Class. Quantum Grav. 32 (2015), no. 12, 124006 .

[100] K. S. Thorne, Relativistic stars, black holes and gravitational waves (including an in-depth review of the theory of rotating, relativistic stars)., General Relativity and Cosmology (R. K. Sachs, ed.), 1971, pp. 237-283.

[101] M. Urbanec, J. C. Miller, and Z. Stuchlik, Quadrupole moments of rotating neutron stars and strange stars, Mon. Not. Roy. Astron. Soc. 433 (2013), 1903.

[102] R. M. Wald, General Relativity, University of Chicago Press, 1984.

[103] _ The thermodynamics of black holes, Living Rev. Rel. 4 (2001), 6.

[104] J. A. Wheeler, Our universe: the known and the unknown, Am. Scholar (1968), $248-274$.

[105] D. L. Wiltshire, M. Visser, and S. M. Scott, The Kerr spacetime: Rotating black holes in general relativity, Cambridge University Press, 2009.

[106] R. P. Woodard, How Far Are We from the Quantum Theory of Gravity?, Rept. Prog. Phys. 72 (2009), 126002. 


\section{Appendix A}

\section{KOMAR INTEGRAL}

For our immediate purpose, we concentrate on the concept of total energy (mass) for an asymptotically flat spacetime. We follow the discussions given in $[14,81]$. A stationary spacetime is characterized for having a timelike Killing vector $K_{\mu}$ at infinity. For this scenario, we can construct the current

$$
J^{\mu}=K_{\nu} R^{\mu \nu},
$$

where $R^{\mu \nu}$ is the Ricci tensor. Using Einstein's equations, (A.1) can be written as

$$
\begin{aligned}
J^{\mu} & =K_{\nu}\left(8 \pi T^{\mu \nu}+\frac{1}{2} R g^{\mu \nu}\right) \\
& =8 \pi K_{\nu}\left(T^{\mu \nu}-\frac{1}{2} T g^{\mu \nu}\right),
\end{aligned}
$$

where we used $T \equiv T_{\mu}^{\mu}$. Taking the divergence of this current we have

$$
\nabla_{\mu} J^{\mu}=\left(\nabla_{\mu} K_{\nu}\right) R^{\mu \nu}+K_{\nu}\left(\nabla_{\mu} R^{\mu \nu}\right) .
$$

Note that $R^{\mu \nu}$ is symmetric but $\nabla_{\mu} K_{\nu}$ is antisymmetric, by virtue of the Killing equation $\nabla_{(\mu} K_{\nu)}=0$, thus the first term to the right of (A.3) vanishes. The divergence of the Ricci tensor can be found to be

$$
\nabla_{\mu} R^{\mu \nu}=\frac{1}{2} \nabla^{\nu} R
$$

Using (A.4) into (A.3) 


$$
\nabla_{\mu} J^{\mu}=\frac{1}{2} K_{\nu} \nabla^{\nu} R=0
$$

where we used the fact that the directional derivative of the Ricci scalar, along a Killing vector, is zero. Thus the current (A.1) is a conserved quantity. In analogy to the electromagnetic case, one can associate a conserved energy to the current $J_{\mu}$

$$
E=\frac{1}{4 \pi} \int_{\Sigma} d^{3} x \sqrt{\gamma} n_{\mu} J^{\mu}
$$

where $\gamma$ is the determinant of the induced metric $\gamma_{i j}$ and $n_{\mu}$ is the normal vector to the spacelike hypersurface $\Sigma$. Note that the value of energy does not depend on the surface $\Sigma$, thus it can be considered a conserved quantity. Moreover, we can express (A.6) as a surface integral via the generalized Stokes's theorem

$$
\int_{\Sigma} d^{n} x \sqrt{|g|} \nabla_{\mu} V^{\mu}=\int_{\partial \Sigma} d^{n-1} y \sqrt{|\gamma|} n_{\mu} V^{\mu}
$$

where $\gamma_{i j}$ is the induced metric on the boundary in coordinates $y^{i}$. Let's recall that a Killing vector satisfies $\nabla_{\mu} \nabla_{\nu} K^{\mu}=K^{\mu} R_{\mu \nu}$, therefore we can write the current (A.1) as

$$
J^{\mu}=\nabla_{\nu}\left(\nabla^{\mu} K^{\nu}\right)
$$

Using (A.7) and (A.8) into (A.6) we can write the total energy as

$$
E=\frac{1}{4 \pi} \int_{\partial \Sigma} d^{2} x \sqrt{\gamma^{(2)}} n_{\mu} \sigma_{\nu} \nabla^{\mu} K^{\nu}
$$

where the integral is evaluated on the induced hypersurface $\partial \Sigma$, a two-sphere at infinity, with metric $\gamma_{i j}^{(2)}$ and normal vector $\sigma^{\mu}$. Equation (A.9) corresponds to the Komar integral and it's associated to the total energy in a stationary spacetime. 


\section{Appendix B}

\section{EINSTEIN TENSOR FOR AXISYMMETRIC SPACETIMES}

The relevant Einstein tensor components $G_{\mu}{ }^{\nu}=R_{\mu}{ }^{\nu}-\frac{1}{2} R \delta_{\mu}{ }^{\nu}$ for a general axisymmetric spacetime are listed below. We use the general form of the metric

$$
d s^{2}=-e^{2 F}(d t)^{2}+e^{2 H}(d r)^{2}+e^{2 Q}(d \theta)^{2}+e^{2 G}[d \phi-\omega(r) d t]^{2}
$$

where $F, H, Q$ are functions of $r$ and $\theta$. The calculations were carried out using the MATHEMATICA notebook available in Hartle's book [34].

$$
\begin{gathered}
4 e^{2[F(r, \theta)+Q(r, \theta)]} G_{1}{ }^{1}=4 e^{2 F(r, \theta)}\left[F^{(0,1)}(r, \theta) e^{2 H(r, \theta)}\left(G^{(0,1)}(r, \theta)-Q^{(0,1)}(r, \theta)\right)\right. \\
+F^{(1,0)}(r, \theta) G^{(1,0)}(r, \theta) e^{2 Q(r, \theta)}+F^{(0,1)}(r, \theta)^{2} e^{2 H(r, \theta)}+F^{(0,2)}(r, \theta) e^{2 H(r, \theta)} \\
+F^{(1,0)}(r, \theta) e^{2 Q(r, \theta)} Q^{(1,0)}(r, \theta)-G^{(0,1)}(r, \theta) e^{2 H(r, \theta)} Q^{(0,1)}(r, \theta)+G^{(0,1)}(r, \theta)^{2} e^{2 H(r, \theta)} \\
\left.+G^{(0,2)}(r, \theta) e^{2 H(r, \theta)}+G^{(1,0)}(r, \theta) e^{2 Q(r, \theta)} Q^{(1,0)}(r, \theta)\right]+\omega^{\prime}(r)^{2} e^{2[G(r, \theta)+Q(r, \theta)])}(\mathrm{B} .2) \\
G_{2}{ }^{1}=F^{(0,1)}(r, \theta)\left[Q^{(1,0)}(r, \theta)-F^{(1,0)}(r, \theta)\right]-G^{(0,1)}(r, \theta) G^{(1,0)}(r, \theta) \\
+H^{(0,1)}(r, \theta)\left[F^{(1,0)}(r, \theta)+G^{(1,0)}(r, \theta)\right]+G^{(0,1)}(r, \theta) Q^{(1,0)}(r, \theta)-F^{(1,1)}(r, \theta)-G^{(1,1)}(r, \theta) \\
+\mathrm{B} .3) \\
4 e^{2[F(r, \theta)+H(r, \theta)]} G_{2}{ }^{2}=4 e^{2 F(r, \theta)}\left\{e^{2 H(r, \theta)} G^{(0,1)}(r, \theta) H^{(0,1)}(r, \theta)\right. \\
+e^{2 H(r, \theta)} F^{(0,1)}(r, \theta)\left(G^{(0,1)}(r, \theta)+H^{(0,1)}(r, \theta)\right)+e^{2 Q(r, \theta)}\left[F^{(1,0)}(r, \theta)^{2}\right. \\
+\left(G^{(1,0)}(r, \theta)-H^{(1,0)}(r, \theta)\right) F^{(1,0)}(r, \theta)+G^{(1,0)}(r, \theta)^{2}-G^{(1,0)}(r, \theta) H^{(1,0)}(r, \theta) \\
\left.\left.+F^{(2,0)}(r, \theta)+G^{(2,0)}(r, \theta)\right]\right\}-\left(\omega^{\prime}\right)^{2} e^{2[G(r, \theta)+Q(r, \theta)]}(\mathrm{B} .4)
\end{gathered}
$$




$$
\begin{aligned}
& 4 e^{2[F(r, \theta)-G(r, \theta)+H(r, \theta)+Q(r, \theta)]} G_{3}{ }^{3}=4 e^{2 F(r, \theta)}\left\{e^{2 H(r, \theta)} F^{(0,1)}(r, \theta)^{2}\right. \\
& +e^{2 H(r, \theta)}\left(H^{(0,1)}(r, \theta)-Q^{(0,1)}(r, \theta)\right) F^{(0,1)}(r, \theta)-F^{(1,0)}(r, \theta) H^{(1,0)}(r, \theta) e^{2 Q(r, \theta)} \\
& +e^{2 H(r, \theta)}\left[H^{(0,1)}(r, \theta)\right]^{2}+e^{2 Q(r, \theta)} F^{(1,0)}(r, \theta)^{2}+e^{2 Q(r, \theta)} Q^{(1,0)}(r, \theta)^{2} \\
& -e^{2 H(r, \theta)} H^{(0,1)}(r, \theta) Q^{(0,1)}(r, \theta)+e^{2 H(r, \theta)} F^{(0,2)}(r, \theta)+e^{2 H(r, \theta)} H^{(0,2)}(r, \theta) \\
& -H^{(1,0)}(r, \theta) e^{2 Q(r, \theta)} Q^{(1,0)}(r, \theta)+e^{2 Q(r, \theta)} F^{(1,0)}(r, \theta) Q^{(1,0)}(r, \theta)+e^{2 Q(r, \theta)} F^{(2,0)}(r, \theta) \\
& \left.+e^{2 Q(r, \theta)} Q^{(2,0)}(r, \theta)\right\}-3\left(\omega^{\prime 2}\right) e^{2[G(r, \theta)+Q(r, \theta)]} \\
& 4 e^{2[F(r, \theta)-G(r, \theta)+H(r, \theta)+Q(r, \theta)]} G_{4}{ }^{3}=2 e^{2(F(r, \theta)+Q(r, \theta))}\left[\omega^{\prime \prime}(r)\right. \\
& \left.-\omega^{\prime}(r)\left(F^{(1,0)}(r, \theta)-3 G^{(1,0)}(r, \theta)+H^{(1,0)}(r, \theta)-Q^{(1,0)}(r, \theta)\right)\right] \\
& +\omega(r)\left[3\left(\omega^{\prime}\right)^{2} e^{2[G(r, \theta)+Q(r, \theta)]}-4 e^{2 F(r, \theta)}\left[e^{2 H(r, \theta)} F^{(0,1)}(r, \theta)^{2}\right.\right. \\
& +e^{2 H(r, \theta)}\left(H^{(0,1)}(r, \theta)-Q^{(0,1)}(r, \theta)\right) F^{(0,1)}(r, \theta)-F^{(1,0)}(r, \theta) H^{(1,0)}(r, \theta) e^{2 Q(r, \theta)} \\
& +e^{2 H(r, \theta)}\left[H^{(0,1)}(r, \theta)\right]^{2}+e^{2 Q(r, \theta)}\left[F^{(1,0)}(r, \theta)\right]^{2}+e^{2 Q(r, \theta)}\left[Q^{(1,0)}(r, \theta)\right]^{2} \\
& -e^{2 H(r, \theta)} H^{(0,1)}(r, \theta) Q^{(0,1)}(r, \theta)+e^{2 H(r, \theta)} F^{(0,2)}(r, \theta)+e^{2 H(r, \theta)} H^{(0,2)}(r, \theta) \\
& -H^{(1,0)}(r, \theta) e^{2 Q(r, \theta)} Q^{(1,0)}(r, \theta)+e^{2 Q(r, \theta)} F^{(1,0)}(r, \theta) Q^{(1,0)}(r, \theta) \\
& \left.\left.+e^{2 Q(r, \theta)} F^{(2,0)}(r, \theta)+e^{2 Q(r, \theta)} Q^{(2,0)}(r, \theta)\right]\right]
\end{aligned}
$$




$$
\begin{gathered}
4 e^{2(F(r, \theta)+H(r, \theta)+Q(r, \theta))} G_{4}{ }^{4}=-e^{2(G(r, \theta)+Q(r, \theta))}\left(3 e^{2 G(r, \theta)}(\omega)^{2}+e^{2 F(r, \theta)}\right)\left(\omega^{\prime}\right)^{2} \\
+4 e^{2(F(r, \theta)+G(r, \theta)+Q(r, \theta))} \omega\left(F^{(1,0)}(r, \theta)-3 G^{(1,0)}(r, \theta)+H^{(1,0)}(r, \theta)-Q^{(1,0)}(r, \theta)\right) \omega^{\prime} \\
-4 e^{2 F(r, \theta)}\left(-e^{2 G(r, \theta)}\left(e^{2 H(r, \theta)}\left[F^{(0,1)}(r, \theta)\right]^{2}+e^{2 H(r, \theta)}\left(H^{(0,1)}(r, \theta)-Q^{(0,1)}(r, \theta)\right) F^{(0,1)}(r, \theta)\right.\right. \\
-F^{(1,0)}(r, \theta) H^{(1,0)}(r, \theta) e^{2 Q(r, \theta)}+e^{2 H(r, \theta)}\left[H^{(0,1)}(r, \theta)\right]^{2}+e^{2 Q(r, \theta)} F^{(1,0)}(r, \theta)^{2} \\
+e^{2 Q(r, \theta)}\left[Q^{(1,0)}(r, \theta)\right]^{2}-e^{2 H(r, \theta)} H^{(0,1)}(r, \theta) Q^{(0,1)}(r, \theta)+e^{2 H(r, \theta)} F^{(0,2)}(r, \theta) \\
+e^{2 H(r, \theta)} H^{(0,2)}(r, \theta)-H^{(1,0)}(r, \theta) e^{2 Q(r, \theta)} Q^{(1,0)}(r, \theta)+e^{2 Q(r, \theta)} F^{(1,0)}(r, \theta) Q^{(1,0)}(r, \theta) \\
\left.+e^{2 Q(r, \theta)} F^{(2,0)}(r, \theta)+e^{2 Q(r, \theta)} Q^{(2,0)}(r, \theta)\right)(\omega)^{2}+e^{2(G(r, \theta)+Q(r, \theta))} \omega^{\prime \prime}(r) \omega(r) \\
+e^{2 F(r, \theta)}\left(e^{2 H(r, \theta)}\left[G^{(0,1)}(r, \theta)\right]^{2}+e^{2 H(r, \theta)}\left(H^{(0,1)}(r, \theta)-Q^{(0,1)}(r, \theta)\right) G^{(0,1)}(r, \theta)\right. \\
-G^{(1,0)}(r, \theta) H^{(1,0)}(r, \theta) e^{2 Q(r, \theta)}+e^{2 H(r, \theta)}\left[H^{(0,1)}(r, \theta)\right]^{2}+e^{2 Q(r, \theta)}\left[G^{(1,0)}(r, \theta)\right]^{2} \\
+e^{2 Q(r, \theta)}\left[Q^{(1,0)}(r, \theta)\right]^{2}-e^{2 H(r, \theta)} H^{(0,1)}(r, \theta) Q^{(0,1)}(r, \theta)+e^{2 H(r, \theta)} G^{(0,2)}(r, \theta) \\
+e^{2 H(r, \theta)} H^{(0,2)}(r, \theta)-H^{(1,0)}(r, \theta) e^{2 Q(r, \theta)} Q^{(1,0)}(r, \theta)+e^{2 Q(r, \theta)} G^{(1,0)}(r, \theta) Q^{(1,0)}(r, \theta) \\
\left.\left.+e^{2 Q(r, \theta)} G^{(2,0)}(r, \theta)+e^{2 Q(r, \theta)} Q^{(2,0)}(r, \theta)\right)\right) \quad(\mathrm{B} .7)
\end{gathered}
$$




\begin{abstract}
Appendix C
INTEGRAL AND SURFACE PROPERTIES OF A SLOWLY ROTATING 'SCHWARZSCHILD STAR' IN GENERAL RELATIVITY
\end{abstract}


Table C.1: Integral and surface properties of a slowly rotating 'Schwarzschild star' for several values of the compactness parameter $R / R_{S}$, where $R$ is the radius of the star and $R_{s}=2 M_{0}$ is the Schwarzschild radius, in the regime $R / R_{S} \geq 9 / 8$. We use geometrized units $(c=G=1)$. The angular velocity relative to the local ZAMO $\varpi(R)=\left.(\Omega-\omega)\right|_{r=R}$ is given in units of $J / R_{s}^{3}$. The moment of inertia $I$ is in the unit $R_{s}^{3}$. The ratio $\delta M^{H} / M$ denotes the original Hartle's fractional change in mass, as given by (3.33), measured in units of $J^{2} / R_{s}^{4}$. The ratio $\delta M / M$ corresponds to the amended fractional change of mass as given by (3.34). The quadrupole moment $Q$ is measured in units of $J^{2} / R_{S}$. The ellipticity $\varepsilon$ is measured in units of $J^{2} / R_{S}^{4}$. All the quantities are computed at the surface of the configuration. The digit in parenthesis following each entry corresponds to the power of ten by which the entry is multiplied.

\begin{tabular}{|c|c|c|c|c|c|c|c|c|}
\hline$R / R_{S}$ & $\varpi(R)$ & $\Omega$ & $I$ & $I_{N}=I / M_{0} R^{2}$ & $\delta M^{H} / M$ & $\delta M / M$ & $Q$ & $\varepsilon$ \\
\hline 100.0 & $4.958538(-4)$ & $4.978538(-4)$ & $2.008621(3)$ & $4.017243(-1)$ & $9.950087(-4)$ & $4.901465(-1)$ & $1.220773(3)$ & $6.157998(-1)$ \\
\hline 50.0 & $1.966802(-3)$ & $1.982802(-3)$ & $5.043366(2)$ & $4.034693(-1)$ & $3.960078(-3)$ & $9.608127(-1)$ & $5.960749(2)$ & $1.213351(0)$ \\
\hline 35.0 & $3.984776(-3)$ & $4.031423(-3)$ & $2.480513(2)$ & $4.049817(-1)$ & $8.046649(-3)$ & $1.348994(0)$ & $4.087341(2)$ & $1.710989(0)$ \\
\hline 20.0 & $1.197994(-2)$ & $1.222994(-2)$ & $8.176653(1)$ & $4.088326(-1)$ & $2.437122(-2)$ & $2.259332(0)$ & $2.216473(2)$ & $2.896803(0)$ \\
\hline 10.0 & $4.582047(-2)$ & $4.782047(-2)$ & $2.091154(1)$ & $4.182308(-1)$ & $9.493460(-2)$ & $4.065297(0)$ & $9.789224(1)$ & $5.352755(0)$ \\
\hline 5.00 & $1.662453(-1)$ & $1.822453(-1)$ & $5.487109(0)$ & $4.389687(-1)$ & $3.588505(-1)$ & $6.482244(0)$ & $3.772876(1)$ & $9.030235(0)$ \\
\hline 4.00 & $2.462305(-1)$ & $2.774805(-1)$ & $3.603856(0)$ & $4.504820(-1)$ & $5.439746(-1)$ & $7.172851(0)$ & $2.635975(1)$ & $1.030041(1)$ \\
\hline 3.00 & $3.969855(-1)$ & $4.710595(-1)$ & $2.122873(0)$ & $4.717497(-1)$ & $9.162101(-1)$ & $7.688984(0)$ & $1.567424(1)$ & $1.165938(1)$ \\
\hline 2.50 & $5.237493(-1)$ & $6.517493(-1)$ & $1.534332(0)$ & $4.909863(-1)$ & $1.258944(0)$ & $7.630176(0)$ & $1.080788(1)$ & $1.213041(1)$ \\
\hline 2.00 & $7.029353(-1)$ & $9.529353(-1)$ & $1.049389(0)$ & $5.246945(-1)$ & $1.819485(0)$ & $6.951236(0)$ & $6.526268(0)$ & $1.195797(1)$ \\
\hline 1.90 & $7.443988(-1)$ & $1.035986(0)$ & $9.652634(-1)$ & $5.347720(-1)$ & $1.971410(0)$ & $6.684018(0)$ & $5.772930(0)$ & $1.176106(1)$ \\
\hline 1.80 & $7.862084(-1)$ & $1.129143(0)$ & $8.856266(-1)$ & $5.466831(-1)$ & $2.140268(0)$ & $6.354842(0)$ & $5.064371(0)$ & $1.147805(1)$ \\
\hline 1.70 & $8.265015(-1)$ & $1.233584(0)$ & $8.106455(-1)$ & $5.610003(-1)$ & $2.327408(0)$ & $5.954579(0)$ & $4.406646(0)$ & $1.108923(1)$ \\
\hline 1.60 & $8.620448(-1)$ & $1.350326(0)$ & $7.405618(-1)$ & $5.785639(-1)$ & $2.533403(0)$ & $5.473614(0)$ & $3.806134(0)$ & $1.056948(1)$ \\
\hline 1.50 & $8.871430(-1)$ & $1.479735(0)$ & $6.757963(-1)$ & $6.007078(-1)$ & $2.756967(0)$ & $4.905143(0)$ & $3.271745(0)$ & $9.893372(0)$ \\
\hline 1.40 & $8.917486(-1)$ & $1.620611(0)$ & $6.170509(-1)$ & $6.296438(-1)$ & $2.992654(0)$ & $4.246032(0)$ & $2.813085(0)$ & $9.034087(0)$ \\
\hline 1.30 & $8.574163(-1)$ & $1.767748(0)$ & $5.656913(-1)$ & $6.694571(-1)$ & $3.224895(0)$ & $3.506898(0)$ & $2.440935(0)$ & $7.971065(0)$ \\
\hline 1.20 & $7.481799(-1)$ & $1.905587(0)$ & $5.247725(-1)$ & $7.288508(-1)$ & $3.412176(0)$ & $2.725004(0)$ & $2.169032(0)$ & $6.728235(0)$ \\
\hline 1.15 & $6.439067(-1)$ & $1.958939(0)$ & $5.104803(-1)$ & $7.719929(-1)$ & $3.454114(0)$ & $2.347975(0)$ & $2.076202(0)$ & $6.090137(0)$ \\
\hline 1.125 & $5.727118(-1)$ & $1.977375(0)$ & $5.057207(-1)$ & $7.991636(-1)$ & $3.442297(0)$ & $2.176744(0)$ & $2.023119(0)$ & $5.531918(0)$ \\
\hline
\end{tabular}


Table C.2: Integral and surface properties of a slowly rotating 'Schwarzschild star' for several values of the deviation parameter $\zeta \equiv \frac{R-R_{s}}{R_{s}}$, where $R$ is the radius of the star and $R_{s}=2 M_{0}$ is the Schwarzschild radius, in the regime $1<R / R_{S}<9 / 8$. We use geometrized units $(c=G=1)$. The angular velocity relative to the local ZAMO $\varpi(R)=\left.(\Omega-\omega)\right|_{r=R}$ is given in units of $J / R_{s}^{3}$. The moment of inertia $I$ is in the unit $R_{s}^{3}$. The ratio $\delta M^{H} / M$ denotes the original Hartle's fractional change in mass, as given by (3.31), measured in units of $J^{2} / R_{s}^{4}$. The ratio $\delta M / M$ corresponds to the amended fractional change of mass as given by (3.34). The ratio $\Delta Q / Q$ defined in (4.7) corresponds to the relative deviation of the mass quadrupole moment from that of the Kerr metric. We measure the quadrupole moment $Q$ in units of $J^{2} / M_{0}$ so the Kerr factor $\bar{q}=Q M_{0} / J^{2}$ corresponds to the unity. The ellipticity $\varepsilon$ is measured in units of $J^{2} / M_{0}^{4}$. All the quantities are computed at the surface of the configuration. The digit in parenthesis following each entry corresponds to the power of ten by which the entry is multiplied.

\begin{tabular}{|c|c|c|c|c|c|c|c|c|}
\hline$\zeta$ & $\varpi(R)$ & $\Omega$ & $\bar{I}$ & $I_{N}=I / M_{0} R^{2}$ & $\delta M^{H} / M$ & $\delta M / M$ & $\Delta Q / Q$ & $\varepsilon$ \\
\hline 0.124 & $5.747365(-1)$ & $1.983152(0)$ & $0.504247(0)$ & $7.982541(-1)$ & $3.410787(0)$ & $2.182713(0)$ & $1.972540(-2)$ & $5.780040(0)$ \\
\hline 0.120 & $5.796710(-1)$ & $2.003231(0)$ & $4.991934(-1)$ & $7.959078(-1)$ & $3.318006(0)$ & $2.239330(0)$ & $1.498300(-2)$ & $3.570681(-1)$ \\
\hline 0.115 & $5.912024(-1)$ & $2.034000(0)$ & $4.916420(-1)$ & $7.909140(-1)$ & $3.146362(0)$ & $2.206228(0)$ & $8.225660(-3)$ & $3.498988(-1)$ \\
\hline 0.114 & $5.928188(-1)$ & $2.039505(0)$ & $4.903149(-1)$ & $7.901959(-1)$ & $3.118945(0)$ & $2.207108(0)$ & $7.163111(-3)$ & $3.488226(-1)$ \\
\hline 0.113 & $5.943907(-1)$ & $2.044980(0)$ & $4.890023(-1)$ & $7.894971(-1)$ & $3.091923(0)$ & $2.207689(0)$ & $6.139552(-3)$ & $3.477814(-1)$ \\
\hline 0.112 & $5.959175(-1)$ & $2.050423(0)$ & $4.877040(-1)$ & $7.888179(-1)$ & $3.065294(0)$ & $2.207959(0)$ & $5.153962(-3)$ & $3.467748(-1)$ \\
\hline 0.111 & $5.973989(-1)$ & $2.055836(0)$ & $4.864200(-1)$ & $580(-1)$ & $3.039057(0)$ & $2.207950(0)$ & $4.205431(-3)$ & $3.458024(-1)$ \\
\hline 0.110 & $5.988341(-1)$ & $2.061216(0)$ & $4.851503(-1)$ & $177(-1)$ & $3.013208(0)$ & $2.207648(0)$ & $3.292989(-3)$ & $3.448637(-1)$ \\
\hline 0.10 & $6.105343(-1)$ & $2.113163(0)$ & $4.732240(-1)$ & $7.821885(-1)$ & $2.775629(0)$ & $2.191611(0)$ & $4.029515(-3)$ & $3.372674(-1)$ \\
\hline $5.0(-2)$ & $5.749893(-1)$ & $2.302664(0)$ & $4.342794(-1)$ & $7.878086(-1)$ & $2.153885(0)$ & $2.105008(0)$ & $8.882128(-3)$ & $3.476065(-1)$ \\
\hline $1.0(-2)$ & $3.175043(-1)$ & $2.258684(0)$ & $4.427355(-1)$ & $8.680238(-1)$ & $1.984771(0)$ & $1.983793(0)$ & $1.475649(-3)$ & $3.873924(-1)$ \\
\hline $5.0(-3)$ & $2.331664(-1)$ & $2.203463(0)$ & $4.538308(-1)$ & $8.986527(-1)$ & $1.990785(0)$ & $1.990653(0)$ & $6.363087(-4)$ & $3.904995(-1)$ \\
\hline $1.0(-3)$ & $1.091218(-1)$ & $2.103133(0)$ & $4.754809(-1)$ & $9.490627(-1)$ & $1.997866(0)$ & $1.997866(0)$ & $1.001120(-4)$ & $3.863623(-1)$ \\
\hline $5.0(-4)$ & $7.045530(-2)$ & $2.067458(0)$ & $4.836856(-1)$ & $9.664047(-1)$ & $1.997955(0)$ & $1.997943(0)$ & $5.726721(-5)$ & $3.832646(-1)$ \\
\hline $1.0(-4)$ & $3.109358(-2)$ & $2.030493(0)$ & $4.924910(-1)$ & $9.847851(-1)$ & $1.999557(0)$ & $1.999555(0)$ & $1.096807(-6)$ & $3.791711(-1)$ \\
\hline $5.0(-6)$ & $6.929922(-3)$ & $2.006899(0)$ & $4.982809(-1)$ & $9.965519(-1)$ & $1.999977(0)$ & $1.999977(0)$ & $5.326670(-7)$ & $3.760089(-1)$ \\
\hline $1.0(-6)$ & $3.097805(-3)$ & $2.003091(0)$ & $4.992282(-1)$ & $9.984544(-1)$ & $1.999995(0)$ & $1.999995(0)$ & $1.060549(-7)$ & $3.754575(-1)$ \\
\hline $5.0(-8)$ & $6.925427(-4)$ & $2.000692(0)$ & $4.998269(-1)$ & $9.996538(-1)$ & $1.999999(0)$ & $1.999999(0)$ & $5.287854(-9)$ & $3.751032(-1)$ \\
\hline $1.0(-12)$ & $3.097091(-6)$ & $2.000003(0)$ & $4.999992(-1)$ & $9.999984(-1)$ & $2.0(0)$ & $2.0(0)$ & $1.056932(-13)$ & $3.750004(-1)$ \\
\hline $1.0(-14)$ & $3.095714(-7)$ & $2.0(0)$ & $4.999999(-1)$ & $9.999998(-1)$ & $2.0(0)$ & $2.0(0)$ & $1.110223(-15)$ & $3.750000(-1)$ \\
\hline
\end{tabular}




\section{Appendix D}

\section{Runge-KutTA MEthods}

\section{D.1 Fourth-order Runge-Kutta method}

In general, ordinary differential equations (ODE) of order $n$

$$
\frac{d y^{(n)}}{d x}=f\left(x, y, y^{\prime}, \ldots, y^{(n-1)}\right)
$$

can be reduced to a set of $n$ coupled first-order differential equations for the functions $y_{i}$, in the form

$$
\frac{d y_{i}}{d x}=f_{i}\left(x, y_{1}, \ldots, y_{n}\right), \quad i=0, \ldots, n
$$

where the $f_{i}$ are known. This property is very useful when we need to apply numerical methods to solve a set of ODE's. Besides the equations, we also need to specify the boundary conditions according to the specific problem we are solving. In our particular case, we are dealing with an initial value problem where the auxiliary conditions, or initial conditions, are specified at some starting point of $x$ (e.g. the center of the configuration).

A method which is conceptually simple and gives approximate solutions is the Euler method. However, due to the fact that it keeps only first order terms in the Taylor expansion, it's very limited, inaccurate and rarely used. A better method is the so-called Runge-Kutta method. In reality this is a set of methods, depending of the order of the expansion, which gives results with various degrees of accuracy. Technically the Euler method is a Runge-Kutta method at first-order. The second 
order method is the so-called midpoint method. In most cases, practitioners rely on the fourth-order Runge-Kutta method (RK4), which provides high accuracy and is easy to implement in the computer. The basic equations of this method are the following $[49,83]$

$$
\begin{aligned}
\mathbf{k}_{0} & =h \mathbf{f}(x, y) \\
\mathbf{k}_{1} & =h \mathbf{f}\left(x+\frac{h}{2}, \mathbf{y}+\frac{\mathbf{k}_{0}}{2}\right), \\
\mathbf{k}_{2} & =h \mathbf{f}\left(x+\frac{h}{2}, \mathbf{y}+\frac{\mathbf{k}_{1}}{2}\right), \\
\mathbf{k}_{3} & =h \mathbf{f}\left(x+h, \mathbf{y}+\mathbf{k}_{2}\right), \\
\mathbf{y}(x+h) & =\mathbf{y}(x)+\frac{1}{6}\left(\mathbf{k}_{0}+2 \mathbf{k}_{1}+2 \mathbf{k}_{2}+\mathbf{k}_{3}\right)
\end{aligned}
$$

where $h$ is the step size, which is kept constant. Note that we are using vector notation (bold letters), thus we can consider a set of $n$ first-order equations ${ }^{1}$. In each step the routine evaluates the derivative four times; one at the starting point, two evaluations at the midpoints $x+h / 2$, and a final one at the ending point. It's worthwhile to remark that the RK4 method is accurate to terms of order $h^{4}$ with an error of order $h^{5}$.

\section{D.2 Adaptive Methods}

The main disadvantage of the standard RK4 method, is that it does not estimate automatically the truncation error ${ }^{2}$. Thus, when we implement the routine, we must guess a reasonable step size $h$ or find it by trial and error. This becomes an issue when the solutions vary drastically in different regions. For example, in intervals

\footnotetext{
${ }^{1}$ In our particular case, Hartle's equations, we had to solve a set of eight coupled first-order differential equations.

${ }^{2}$ The truncation error is due to the terms omitted in the Taylor series expansion and is given by $E=\frac{1}{(m+1) !} y^{(m+1)}(\xi) h^{(m+1)}$, where $x<\xi<x+h$.
} 
where the function is smooth we might choose a big step size so we can calculate the solution faster. On the other hand, in regions where the function varies rapidly, we might need to consider points which are closer (smaller $h$ ).

The adaptive step size methods provide a technique that estimate the truncation error in each step, and adjust the step size to maintain the error within some predetermined limit. The adaptive method uses the so-called embededd integration formulas, or Runge-Kutta-Fehlberg formulas, which are given by [49, 83]

$$
\begin{aligned}
& \mathbf{k}_{0}=h \mathbf{f}(x, y), \\
& \mathbf{k}_{i}=h \mathbf{f}\left(x+a_{i} h, \mathbf{y}+\sum_{j=0}^{i-1} b_{i j} \mathbf{k}_{j}\right), \quad i=1,2, \ldots, 5 \\
& \mathbf{y}_{5}(x+h)=\mathbf{y}(x)+\sum_{i=0}^{5} c_{i} \mathbf{k}_{i} \quad \text { (fifth-order formula) } \\
& \mathbf{y}_{4}(x+h)=\mathbf{y}(x)+\sum_{i=0}^{5} d_{i} \mathbf{k}_{i} \quad \text { (fourth-order formula). }
\end{aligned}
$$

The coefficients in (D.4) are chosen conventionally to be those proposed by Cash and Karp (see, e.g., [83], p. 717). We used these coefficients in our code. The truncation error is estimated by the relation

$$
\mathbf{E}(h)=\mathbf{y}_{5}(x+h)-\mathbf{y}_{4}(x+h)=\sum_{i=0}^{5}\left(c_{i}-d_{i}\right) \mathbf{k}_{i},
$$

where $E(h)$ scales as $h^{5}$. Note that $\mathbf{E}(h)$ is defined as a "vector", where each component corresponds to the errors in the functions $y_{i}$. In order to control the error measure $e(h)$, it is conventional to choose the root-mean-square value given by [49]

$$
\bar{E}(h)=\sqrt{\frac{1}{n} \sum_{i=0}^{n-1} E_{1}^{2}(h)},
$$

where $n$ is the number of differential equations. In our code we used $e(h)=\bar{E}(h)$ as the error measure. As we discussed above, the idea of the adaptive method is to

\footnotetext{
${ }^{3}$ This definition of vector must be understood, only, from the computational point of view.
} 
adjust the step $h$ such that the error $e(h)$ lies within some predetermined tolerance $\tau$. In order to relate $e(h)$ with the tolerance $\tau$, let's suppose that a step $h_{1}$ produced an error $e_{1}$; considering that the truncation error is of the order $h^{5}$, the step $h_{2}$ which we should have used to obtain an error $e_{2}$ is

$$
h_{2} \approx h_{1}\left(\frac{e_{2}}{e_{1}}\right)^{1 / 5}=h_{1}\left(\frac{\tau}{e_{1}}\right)^{1 / 5} .
$$

where we have chosen $e_{2}=\tau$ as the predetermined tolerance. Equation (D.7) works in the following way: if $e_{1}<\tau$, or equivalently $h_{2}>h_{1}$, the error is below the tolerance therefore the step is good and we use it in the next computation. On the other hand, if $h_{2}<h_{1}$ the error is above the tolerance and the equation tells us how much we should decrease the step-size in the next computation. Note that the estimation of the error is only approximate. Thus it is prudent to introduce a small safety factor, a little less than the unity, in (D.7)

$$
h_{2}=0.9 h_{1}\left(\frac{\tau}{e_{1}}\right)^{1 / 5}
$$

We implemented this equation in our code to adjust the step-size, with a tolerance value of $\tau=1.0 \times 10^{-6}$.

The interested reader can find the routines of the Runge-Kutta-Fehlberg method for Python in the book of Kiusalaas [49]. For the reader familiar with the language C, the classical book by Press et al., [83] provides several routines of the method with error control. 